University of Tennessee Health Science Center UTHSC Digital Commons

\title{
The Role of MCL-1 in the Heart: Gateway from Life to Death
}

\author{
Xi Wang \\ University of Tennessee Health Science Center
}

Follow this and additional works at: https://dc.uthsc.edu/dissertations

Part of the Cardiovascular Diseases Commons, and the Medical Genetics Commons

\section{Recommended Citation}

Wang, Xi , "The Role of MCL-1 in the Heart: Gateway from Life to Death" (2014). Theses and Dissertations (ETD). Paper 368. http://dx.doi.org/10.21007/etd.cghs.2014.0342.

This Dissertation is brought to you for free and open access by the College of Graduate Health Sciences at UTHSC Digital Commons. It has been accepted for inclusion in Theses and Dissertations (ETD) by an authorized administrator of UTHSC Digital Commons. For more information, please contact jwelch30@uthsc.edu. 


\title{
The Role of MCL-1 in the Heart: Gateway from Life to Death
}

\author{
Abstract \\ MCL-1 is an essential BCL-2 family member that promotes the survival of multiple cellular lineages, but its \\ role in cardiac muscle has remained unclear. Here, we have demonstrated that cardiac-specific ablation \\ of $\mathrm{Mcl}-1$ results in a rapidly fatal, dilated cardiomyopathy preceded by loss of myofibrils and cardiac \\ contractility, abnormal mitochondria ultrastructure, defective mitochondrial respiration, and impaired \\ autophagy. Genetic ablation of both pro-apoptotic effectors (Bax and Bak) could largely rescue the \\ lethality and impaired cardiac function induced by Mcl-1 deletion. However, Mcl-1-, Bax-, and Bak-deficient \\ hearts still revealed mitochondrial ultrastructural abnormalities and displayed deficient mitochondrial \\ respiration, and are hypersensitive to chronic isoproterenol challenge. Together, these studies indicate \\ that MCL-1 functions beyond merely blocking cardiomyocyte death and suggest that in myocardium, \\ MCL-1 also facilitates normal mitochondrial function. These findings are important, as overexpression \\ MCL-1 in the heart could be a potential strategy to overcome cardiac injury and promote efficient \\ mitochondrial function and would be beneficial to human health. Of equal importance, specific \\ MCL-1-inhibiting therapeutics that has been proposed to treat cancer may result in unexpected cardiac \\ toxicity; it may be necessary to take this possible side effect into account during MCL-1 inhibitor \\ development. \\ Document Type \\ Dissertation \\ Degree Name \\ Doctor of Philosophy (PhD) \\ Program \\ Biomedical Sciences \\ Research Advisor \\ Joseph T. Opferman, Ph.D. \\ Keywords \\ apoptosis, BCL-2, heart failure, MCL-1, mitochondria \\ Subject Categories \\ Cardiovascular Diseases | Diseases | Medical Genetics | Medical Sciences | Medicine and Health Sciences \\ Comments \\ One year embargo expired December 2015
}


The Role of MCL-1 in the Heart: Gateway from Life to Death

\author{
A Dissertation \\ Presented for \\ The Graduate Studies Council \\ The University of Tennessee \\ Health Science Center \\ In Partial Fulfillment \\ Of the Requirements for the Degree \\ Doctor of Philosophy \\ From The University of Tennessee
}

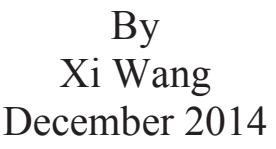


Chapters 2 and 3 (C) 2013 by Cold Spring Harbor Laboratory.

All other material (c) 2014 by Xi Wang.

All rights reserved. 


\section{DEDICATION}

This dissertation is dedicated to every child at Shanghai Children's Medical Center and St. Jude Children's Research Hospital fighting cancer, whose courage and strength inspire me to make the world a better place. 


\section{ACKNOWLEDGEMENTS}

I would like to acknowledge all the people who have helped and supported me throughout my graduate studies. Firstly, I would like to express my deepest gratitude to my advisor Dr. Joseph Opferman, for bringing me to the lab, and all the guidance, caring, patience, mentorship, and providing me with an excellent atmosphere for doing research throughout my graduate studies. I would also thank everyone in the Opferman laboratory, especially the former graduate student Rhonda Perciavalle, whose hard working provides a solid theoretical foundation for my project, Madhavi Bathina, Brian Koss, Dr. Christopher Mill, Dr. Katherine Szarama and Xue Yang for technical assistance and helpful suggestions.

I would like to thank my committee members, Dr. Richard W. Kriwacki, Dr. Peter J. McKinnon, Dr. Rennolds S. Ostrom and Dr. J. Paul Taylor, for their direction, dedication, and invaluable advice along this project for the past several years. Thank them for encouraging and helping me to develop my knowledge and background in biology and biochemistry.

I would like to thank Dr. Christopher Calabrese, Melissa Johnson from the small animal imaging center, Dr. Sharon Frase from the electron microscopy facility, and Dr. Jerold Rehg from the pathology facility for all the technical supports along the way. I would like to thank my collaborators Dr. John Schuetz and Dr. John Lynch. Additionally, I would like to acknowledge the Department of Biochemistry for all the feedbacks in my department meeting over the years.

Finally, I would like to thank my parents for bringing me to this world, raising me up, supporting my education, and most importantly giving me love all the time. I also want to thank my loving husband, Chi Zhang, for his encouragement and support during the past five years, making my life in a foreign country more meaningful and happy. 


\begin{abstract}
MCL-1 is an essential BCL-2 family member that promotes the survival of multiple cellular lineages, but its role in cardiac muscle has remained unclear. Here, we have demonstrated that cardiac-specific ablation of $\mathrm{Mcl}-1$ results in a rapidly fatal, dilated cardiomyopathy preceded by loss of myofibrils and cardiac contractility, abnormal mitochondria ultrastructure, defective mitochondrial respiration, and impaired autophagy. Genetic ablation of both pro-apoptotic effectors (Bax and Bak) could largely rescue the lethality and impaired cardiac function induced by $\mathrm{Mcl}-1$ deletion. However, Mcl-1-, $B a x$-, and Bak-deficient hearts still revealed mitochondrial ultrastructural abnormalities and displayed deficient mitochondrial respiration, and are hypersensitive to chronic isoproterenol challenge. Together, these studies indicate that MCL-1 functions beyond merely blocking cardiomyocyte death and suggest that in myocardium, MCL-1 also facilitates normal mitochondrial function. These findings are important, as overexpression MCL-1 in the heart could be a potential strategy to overcome cardiac injury and promote efficient mitochondrial function and would be beneficial to human health. Of equal importance, specific MCL-1-inhibiting therapeutics that has been proposed to treat cancer may result in unexpected cardiac toxicity; it may be necessary to take this possible side effect into account during MCL-1 inhibitor development.
\end{abstract}




\section{TABLE OF CONTENTS}

CHAPTER 1. INTRODUCTION ..................................................................................

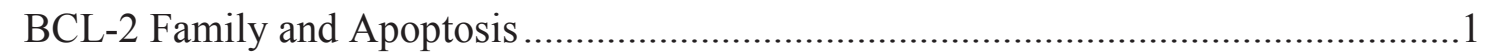

Dual Functions of MCL-1 ...................................................................................

Anti-apoptotic MCL-1 and Cell Survival .........................................................

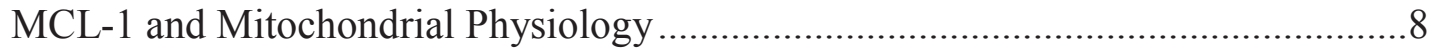

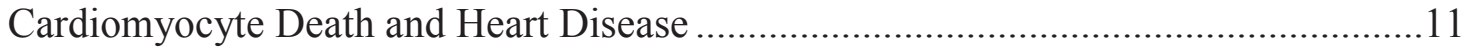

Apoptosis and Heart Disease ................................................................................ 12

Necrosis and Heart Disease................................................................................. 16

Autophagy-associated Cell Death and Heart Disease........................................... 17

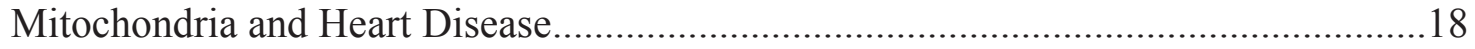

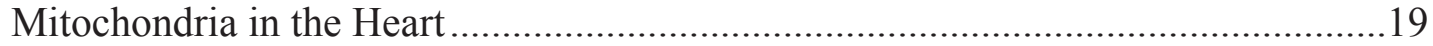

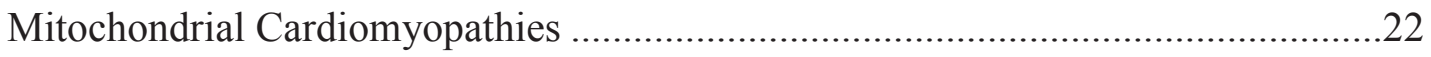

Mitochondria and Heart Failure ......................................................................23

CHAPTER 2. MATERIALS AND EXPERIMENTAL PROCEDURES ...................25

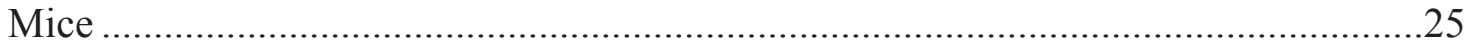

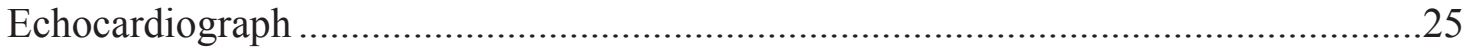

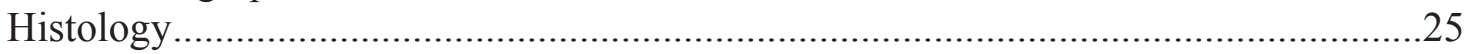

Western Blotting and Antibodies............................................................................26

Isolation and Purification of Mouse Heart Mitochondria ...........................................26

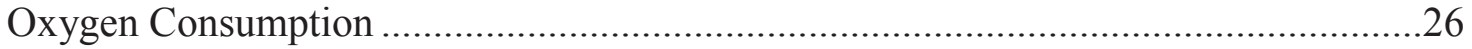

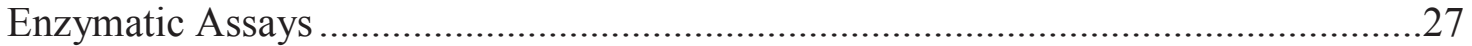

DNA Isolation and Quantification .......................................................................2

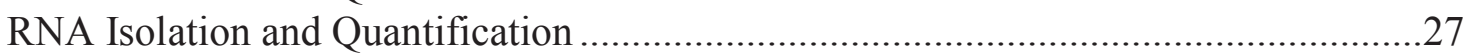

Transmission Electron Microscope .......................................................................28

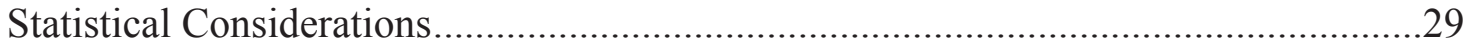

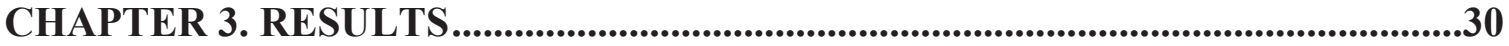

Cardiomyopathy Induced upon Constitutive $\mathrm{Mcl}$-1-deletion in Cardiac and Skeletal

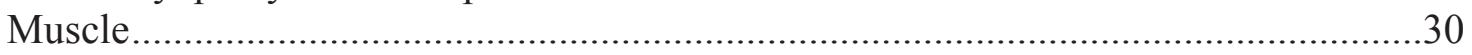

Mcl-1-deletion in Adult Cardiac Muscle Results in Rapid, Fatal Cardiomyopathy .......30

Cardiac-specific Deletion of MCL-1 Induced Cell Death ..........................................34

Mitochondrial Ultrastructural Abnormalities and Respiratory Deficiency in $\mathrm{Mcl}-1$ -

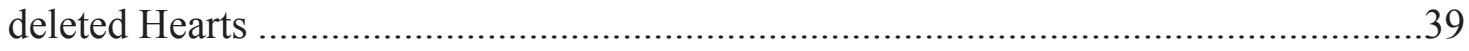

Genetic Ablation of Bax and Bak Improves Cardiac Function and Survival of

Cardiac-specific $\mathrm{Mcl}$-1-deletion

Genetic Ablation of Bax and Bak Is Insufficient to Restore Mitochondrial

Abnormalities Induced by $\mathrm{Mcl}$-1-deletion

Mcl-1-loss in Cardiomyocytes Impairs Autophagy Induction While Co-deletion of

Mcl-1, Bax, and Bak Restores Cardiac Autophagy..... 


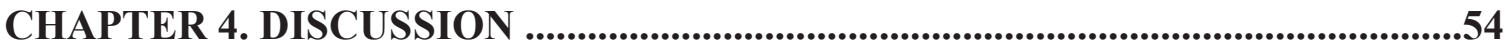

Role of MCL-1 in the Heart Homeostasis and Heart Disease Treatment......................54

BCL-2 Family Members and Cardiomyocyte Death ...................................................54

MCL-1 Is an Essential Pro-survival Regulator for Heart Homeostasis ......................55

MCL-1 and Cardiac Mitochondria Physiology.......................................................57

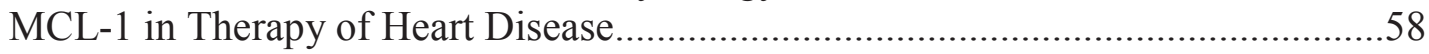

MCL-1 as a Target for Cancer Therapy..................................................................60

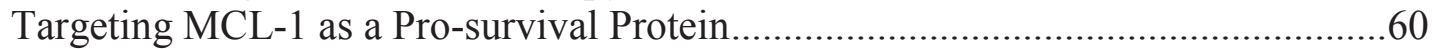

Targeting MCL-1 in Mitochondrial Physiology ...........................................................60

Potential Cardiac Complications of Targeting MCL-1 in Cancer Therapy ................62

CHAPTER 5. FUTURE DIRECTIONS ......................................................................63

Contributions of Different MCL-1 Isoforms to Cardiac Function in vivo ......................63

Reconstitution of $\mathrm{Mcl}$-1-deleted Hearts by Adeno-associated Virus Delivery...............63

Cardiac Function of TKO Mice under Stress …………...............................................69

LIST OF REFERENCES .......................................................................................................72

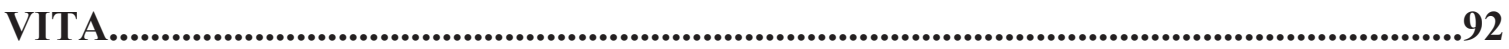




\section{LIST OF TABLES}

Table 3-1. Analysis of the progeny of Ckmm-Cre-mediated deletion of $\mathrm{Mcl}-1$............31

Table 3-2. Oxygen consumption of isolated cardiac mitochondria .............................42

Table 3-3. Quantification of mitochondrial DNA from isolated cardiac muscle ..........45 


\section{LIST OF FIGURES}

Figure 1-1. Scheme depicting intrinsic and extrinsic pathways of apoptosis ..................2

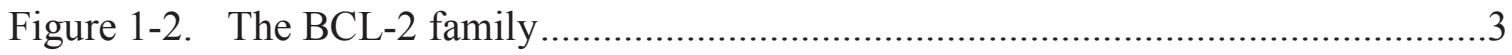

Figure 1-3. Role of anti-apoptotic regulators during hematopoiesis ............................

Figure 1-4. Model for MCL-1's potential functions in mitochondria ............................ 10

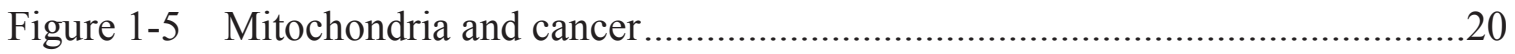

Figure 3-1. Cardiomyopathy is induced upon constitutive $\mathrm{Mcl}-1$ deletion in cardiac

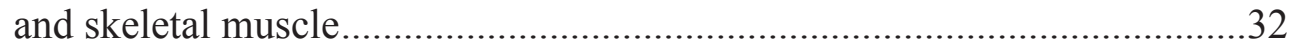

Figure 3-2. $\quad$ Mcl-1-deleted skeletal muscle revealed no overt evidence of myopathy .....33

Figure 3-3. Mcl-1 deletion in adult cardiac muscle results in rapid, fatal

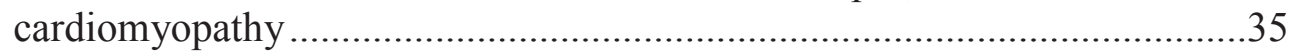

Figure 3-4. Kinetic analyses of cardiac dysfunction in $\mathrm{Mcl}$-1-deleted mice ...................37

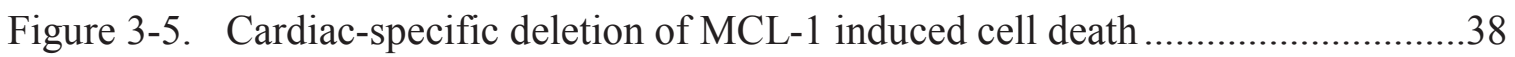

Figure 3-6. Expression of pro-apoptotic molecules in the cardiac mitochondria...........40

Figure 3-7. Mitochondrial ultrastructural abnormalities and respiratory deficiency in

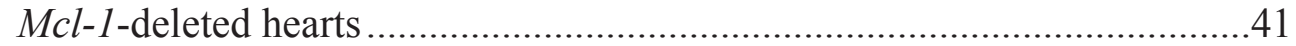

Figure 3-8. Enzymatic function of $\mathrm{Mcl}$-1-deleted mitochondria ...............................44

Figure 3-9. mRNA level of PGC1-alpha and its target genes ....................................46

Figure 3-10. Genetic ablation of Bax and Bak improves cardiac function and survival of constitutive cardiac-specific $\mathrm{Mcl}-1$ deletion .........................................47

Figure 3-11. Genetic ablation of Bax and Bak improves cardiac function and survival of inducible cardiac-specific $\mathrm{Mcl}-1$ deletion.

Figure 3-12. Genetic ablation of Bax and Bak is insufficient to obviate mitochondrial abnormalities induced by $\mathrm{Mcl}$-1-deletion.

Figure 3-13. Mitochondrial ultrastructural abnormalities in $\mathrm{Mcl}$-1-deleted hearts cannot be prevented by genetic ablation of Bax and Bak

Figure 3-14. Mcl-1-loss in cardiomyocytes impairs autophagy induction while codeletion of Mcl-1, Bax, and Bak restores cardiac autophagy .... 
Figure 4-1. MCL-1 resides in different submitochondrial localizations in the heart ......59

Figure 5-1. Delivery AAV constructs to the heart...................................................64

Figure 5-2. Normal cardiac function needs both isoforms of MCL-1 ..........................66

Figure 5-3. Schematic illustration of 2A peptide-linked multicistronic cassettes ..........68

Figure 5-4. Isoproterenol challenge induces cardiac dilation and contractile dysfunction in $M y h$-TKO heart ..................................................... 70

Figure 5-5. MCL-1 is essential for cardiac homeostasis ........................................ 71 


\section{LIST OF ABBREVIATIONS}

\begin{tabular}{ll} 
B-TrCP & B-transducin repeat-containing protein \\
$\Delta$ & Delta \\
$\mu 1$ & Microliter \\
$\mu \mathrm{M}$ & Micromolar \\
A1 & BCL-2 related protein A1 \\
AAV & Adeno-associated virus \\
ADP & Adenosine diphosphate \\
AIF & Apoptosis inducing factor \\
ALL & Acute lymphoblastic leukemia \\
AML & Acute myelogenous leukemia \\
AMPK & Monophosphate-activated protein kinase \\
ANT & Adenine nucleotide translocator \\
APAF-1 & Apoptotic protease activating factor-1 \\
ATP & Adenosine triphosphate \\
BAD & BCL-2 antagonist of cell death \\
BAK & BCL-2 antagonist/killer \\
BAX & BCL-2-associated protein \\
BCL-2 & B cell lymphoma 2 \\
BCL-w & BCL-2-like protein 2 \\
BCL-X & BCL-2-like protein 1 \\
BH & BCL-2 homology \\
BID & BH3-interacting-domian death agonist \\
BIM & BCL-2-interacting mediator of cell death \\
BNIP3 & BCL2/adenovirus E1B 19kD-interacting protein 3 \\
BOK & BCL-2-related ovarian killer \\
BSA & Bovine serum albumin \\
CDK & Cyclin dependent kinase \\
CKMM & Creatine kinase \\
CLL & Chronic lymphocytic leukemia \\
CLP & Common lymphoid progenitor \\
CML & Chronic myelogenous leukemia \\
CMP & Common myeloid progenitor \\
CoA & Coenzyme A \\
CoQ & Coenzyme Q \\
COX & Cytochrome-c oxidase \\
C-terminal & Carboxyl-terminal \\
DMSO & Dimethyl sulfoxide \\
DNA & Deoxyribonucleic acid \\
DRP1 & Dynamin-related protein \\
DTT & Dithiothreitol \\
E & Embryonic day \\
ECHO & Echocardiograph \\
EDTA & Ethylene diaminetetraacetic acid \\
& \\
\hline
\end{tabular}




$\begin{array}{ll}\text { EF } & \text { Ejection fraction } \\ \text { EGTA } & \text { Ethylene glycol bis-(2-amino-ethylether)-N, N, N', N'-tetraacetic } \\ \text { ER } & \text { Endoplasmic reticulum } \\ \text { ERK } & \text { Extracellular signal-regulated kinase } \\ \text { ETC } & \text { Electron transport chain } \\ \text { FAD } & \text { Flavin adenine dinucleotide } \\ \text { FADD } & \text { Fas-associated death domain } \\ \text { FBW7 } & \text { F-box and WD repeat domain containing 7, E3 ubiquitin protein } \\ \text { FCCP } & \text { Carbonycyande-p-trifluoromethoxyphenylhydrazone } \\ \text { FS } & \text { Fraction shortening } \\ \text { GFP } & \text { Green fluorescent protein } \\ \text { GM-CSF } & \text { Granulocyte-macropahge colony-stimulating factor } \\ \text { GMP } & \text { Granulocyte monocyte progenitor } \\ \text { GSK-3 } & \text { Glycogen synthase kinase 3 } \\ \text { HECT } & \text { Homologous to the E6-AP carboxyl terminus } \\ \text { HIF } & \text { Hypoxia induced factor } \\ \text { HM } & \text { Heavy membrane } \\ \text { HSC } & \text { Hematopoietic stem cell } \\ \text { IL } & \text { Interleukin } \\ \text { IMM } & \text { Inner mitochondrial membrane } \\ \text { IMS } & \text { Inner membrane space } \\ \text { IVS } & \text { Interventicular septum } \\ \text { JAK } & \text { Janus kinase } \\ \text { JNK } & \text { c-Jun NH(2)-terminal kinases } \\ \text { kD } & \text { Kilodalton } \\ \text { LCK } & \text { Lymphocyte-specific protein tyrosine kinase } \\ \text { LV } & \text { Left ventricle } \\ \text { LVID } & \text { Left ventricle interior dimension } \\ \text { LVPW } & \text { Left ventricle posterior wall } \\ \text { MAP } & \text { Mitogen-activated protein } \\ \text { MCL-1 } & \text { Myeloid cell leukemia 1 } \\ \text { MEF } & \text { Mouse embryonic fibroblast } \\ \text { MEP } & \text { Micotinamide adenine dinucleotide } \\ \text { MFN } & \text { Megakaryocyte erythroid progenitor } \\ \text { MgCl } & \text { Mitofusin } \\ \text { MIB } & \text { Magnesium chloride } \\ \text { mM } & \text { Mitochondrial isolation buffer } \\ \text { MnSOD } & \text { Minimolar } \\ \text { MOMP } & \text { Manganese superoxide dismutase } \\ \text { MPP } & \text { Mitochondrial outer membrane permeabilization } \\ \text { mPTP } & \text { Matrix processing peptidase } \\ \text { mtDNA } & \text { Mitochondrial permeability transition pore } \\ \text { MTS } & \text { Mitochondrial DNA } \\ \text { MULE } & \text { MYH } \\ \text { NAD } & \end{array}$




$\begin{array}{ll}\text { NADPH } & \text { Nicotinamide adenine dinucleotide phosphate } \\ \text { NCX } & \text { Sodium calcium exchanger } \\ \text { nDNA } & \text { Nuclear DNA } \\ \text { NK } & \text { Natural killer } \\ \text { OMM } & \text { Outer mitochondrial membrane } \\ \text { OPA-1 } & \text { Optic atrophy-1 } \\ \text { OXPHOS } & \text { Oxidative phosphorylation } \\ \text { P } & \text { Postnatal day } \\ \text { PCNA } & \text { Proliferating cell nuclear antigen } \\ \text { PDH } & \text { Pyruvate dehydrogenase } \\ \text { PI3K } & \text { Phosphoinsitide 3 kinase } \\ \text { PUMA } & \text { P53-upregulated modulator of apoptosis } \\ \text { PVDF } & \text { Polyvinylidene fluoride } \\ \text { RNA } & \text { Ribonucleic acid } \\ \text { ROS } & \text { Reactive oxygen species } \\ \text { SC } & \text { Supercomplex } \\ \text { SCF } & \text { Stem cell factor } \\ \text { SDH } & \text { Succinate dehydrogenase } \\ \text { SDS-PAGE } & \text { Sodium dodecyl sulfate poyacryamide gel electrophoresis } \\ \text { Ser } & \text { Serine } \\ \text { SV40 } & \text { Simian virus 40 } \\ \text { TCA } & \text { Tricarboxylic acid } \\ \text { TEM } & \text { Transmission electron microscopy } \\ \text { TFAM } & \text { Mitochondrial transcription factor } \\ \text { TIM } & \text { Translocase of inner membrane } \\ \text { TNF } & \text { Tumor necrosis factor } \\ \text { TNFR } & \text { Tumor necrosis factor receptor } \\ \text { TNFR1 } & \text { Tumor necrosis factor receptor-1 } \\ \text { TOM } & \text { Translocase of outer membrane } \\ \text { TPA } & \text { 12-O-tetradecanoylphorbol-13-acetate } \\ \text { TUNEL } & \text { Terminal deoxynucleotidyl transferase-mediated dUTP nick end } \\ \text { UPR } & \text { labeling } \\ \text { USP9X } & \text { Unfolded protein response } \\ \text { UV } & \text { Uliquitin specific peptidase } 9, \text { X-linked } \\ \text { VDAC } & \text { Voltagendent anion channel } \\ & \end{array}$




\section{CHAPTER 1. INTRODUCTION}

\section{BCL-2 Family and Apoptosis}

Apoptosis is an essential part of the life cycle for many organisms, and a fundamental mechanism for homeostasis of tissues. Dysfunction of apoptosis leads to a variety of human pathologies including cancer, neurodegenerative, and autoimmune diseases (Cory and Adams 2002). Apoptosis is initiated when death stimuli trigger multiprotein complexes that recruit and activate initiator caspases. There are two cell death pathways requiring the activation of initiator caspases (by multi-protein complexes), extrinsic and intrinsic apoptosis pathway (Munoz-Pinedo 2012) (Figure 1-1).

In the extrinsic apoptosis pathway, death stimuli interact with death receptors on the surface of the cell. The death receptors contain an intracellular death domain that recruits and activates initiator Caspase- 8 . Once activated, Caspase- 8 activates effector caspases, such as Caspase-3, which cleave a number of cellular components to induce cell death (Hengartner 2000) (Figure 1-1). In many cell types, the extrinsic pathway for cell death does not involve BCL-2 family members or mitochondrial outer membrane permeabilization (MOMP) to amplify the death signal.

On the other hand, the intrinsic apoptosis pathway is regulated by BCL-2 family members on the mitochondria. BCL-2 family members can be divided into two groups according to their function: pro-apoptotic members and anti-apoptotic members. BCL-2 family members share homology within four conserved amphipathic alpha-helical segments called BCL-2 homology domains (BH) (Youle and Strasser 2008). The antiapoptotic members (such as BCL-2, BCL-X $\mathrm{L}, \mathrm{MCL}-1, \mathrm{~A} 1$ and BCL-w) possess multiple $\mathrm{BH}$ domains and form a hydrophobic pocket that allows these members to interact with the $\mathrm{BH} 3$ domain of other pro-apoptotic family members to perform the anti-apoptotic function. The pro-apoptotic members can be further divided into two subgroups depending on the number of $\mathrm{BH}$ domains; multi-domain pro-apoptotic effectors (such as $\mathrm{BAX}, \mathrm{BAK}$ and $\mathrm{BOK}$ ) and $\mathrm{BH} 3$ only pro-apoptotic members (such as BID, BAD, BIM, BIK, NOXA, PUMA, etc.) (Cheng et al. 2001; Opferman and Korsmeyer 2003; Petros et al. 2004) (Figure 1-2). BH3 only members reside upstream of the intrinsic apoptosis pathway, and can be activated by different death stimuli to promote the activation and oligomerization of BAX and BAK. BAX and BAK oligomers permeablize the outer mitochondrial membrane (OMM) and facilitate the release of several apoptogenic proteins including cytochrome $c$. Cytochrome $c$ then binds to apoptotic proteaseactivating factor-1 (APAF-1) to induce a conformational change, so that APAF-1 can recruit initiator Caspase-9. APAF-1 bound Caspase-9 can then activate itself through dimerization. The activated Caspase- 9 then activates effector caspases, which cleave a myriad of intracellular targets, to induce cell death (Gross et al. 1999) (Figure 1-1). 


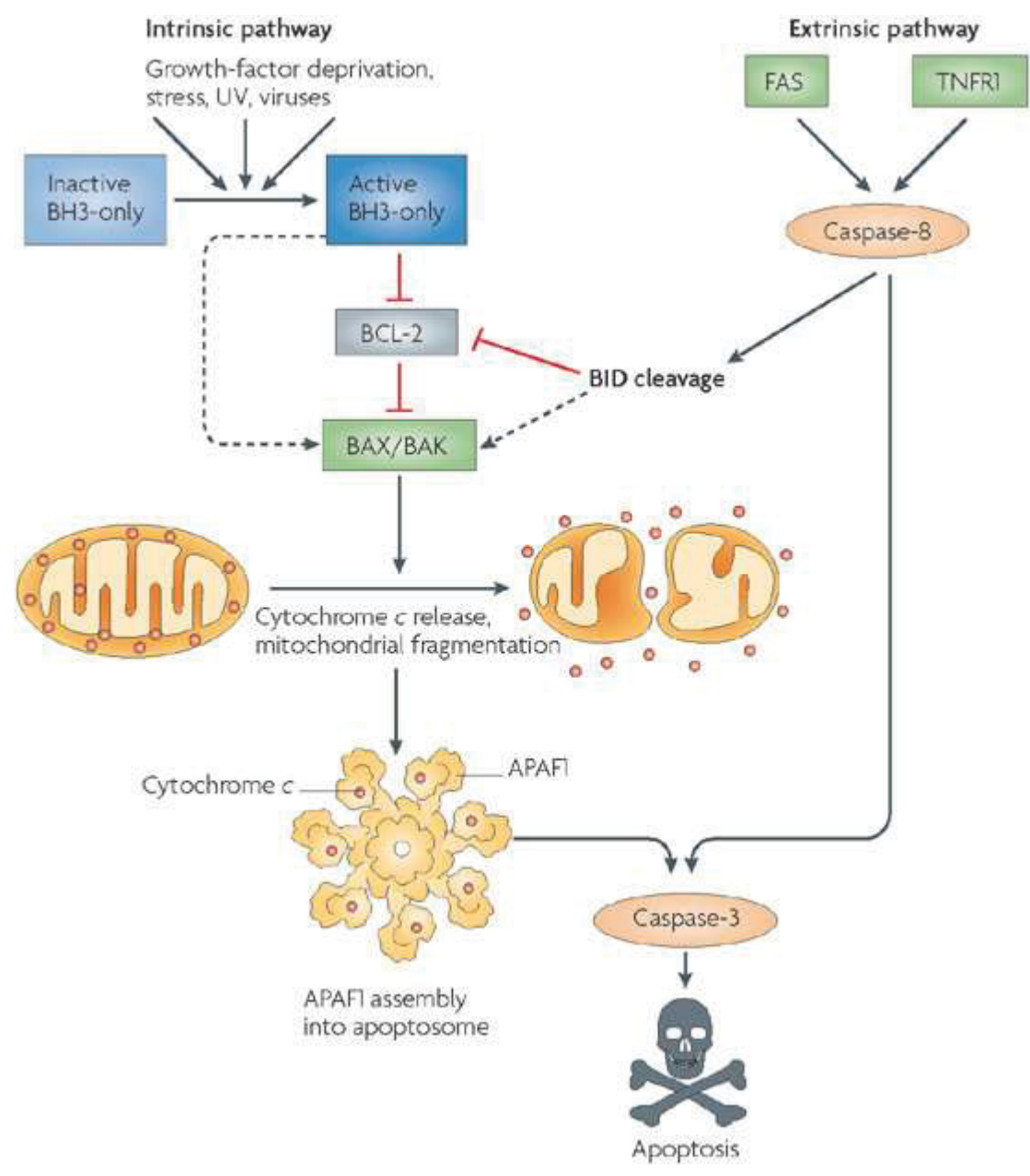

Figure 1-1. Scheme depicting intrinsic and extrinsic pathways of apoptosis Apoptosis can be induced by cell surface receptors, such as Fas and tumor necrosis factor receptor-1 (TNFR1) (extrinsic pathway, right), or by various genotoxic agents, metabolic insults or transcriptional cues (intrinsic pathway, left). The intrinsic pathway starts with BH3-only protein induction or post-translational activation, which results in the inactivation of some BCL-2 family members. This relieves inhibition of BAX and BAK activation, which in turn promotes apoptosis. Some BH3-only proteins, such as BIM and PUMA, may also be able to activate BAX and/or BAK (as shown by the dotted line). Once activated, BAX and BAK promote cytochrome $c$ release and mitochondrial fragmentation, which leads to the activation of APAF1 into an apoptosome and activates Caspase-9 to activate Caspase-3. Caspases in turn cleave a series of substrates, activate DNases and orchestrate the demolition of the cell. The extrinsic pathway can bypass the mitochondrial step and activate caspase- 8 directly, which leads to Caspase- 3 activation and cell demolition. The BCL-2 family regulates the intrinsic pathway and can modulate the extrinsic pathway when cleavage of BID communicates between the two pathways. Reprinted with permission from Macmillan Publishers Ltd: Youle RJ, Strasser A. 2008. The BCL-2 protein family: opposing activities that mediate cell death. Nat Rev Mol Cell Biol 9: 47-59. 
Figure 1-2. The BCL-2 family

The BCL-2 family compromises pro-apoptotic and anti-apoptotic members. BCL-2 family members share homology within four conserved $\alpha$-helical segments, called $\mathrm{BH}$ domains. The anti-apoptotic BCL-2 family members (BCL-2, BCL-X $\mathrm{L}, \mathrm{BCL}-\mathrm{W}$ and A1) possess multiple $\mathrm{BH}$ domains, of which the $\mathrm{BH} 1-\mathrm{BH} 3$ domains form a hydrophobic pocket capable of binding the $\mathrm{BH} 3$ domain of the other pro-apoptotic family members. Boo/Diva is a mouse BCL-2 homology that is highly expressed in the ovary, but only weakly in other tissues. NR13 is the avian cell death antagonist shares homology with Boo/Diva. The pro-apoptotic family members can be subdivided into two groups according to the number of $\mathrm{BH}$ domains they possess. The multi-domain pro-apoptotic members (BAX, BAK and $\mathrm{BOK}$ ) possess the $\mathrm{BH} 1, \mathrm{BH} 2$ and $\mathrm{BH} 3$ domains. In contrast, the $\mathrm{BH} 3$-only members contain only the $\mathrm{BH} 3$ minimal death domain. Reprinted with permission from Macmillan Publishers Ltd: Opferman JT, Korsmeyer SJ. 2003. Apoptosis in the development and maintenance of the immune system. Nature Immunology 4: 410-415. 


\section{Anti-apoptotic}

$\mathrm{Bcl}-2$

Mammalian

$\mathrm{Bcl}-\mathrm{x}_{\mathrm{L}}$

Bcl-w

Mcl-1

A1/Bfl-1

Boo/Diva

$\mathrm{Nr}-13$

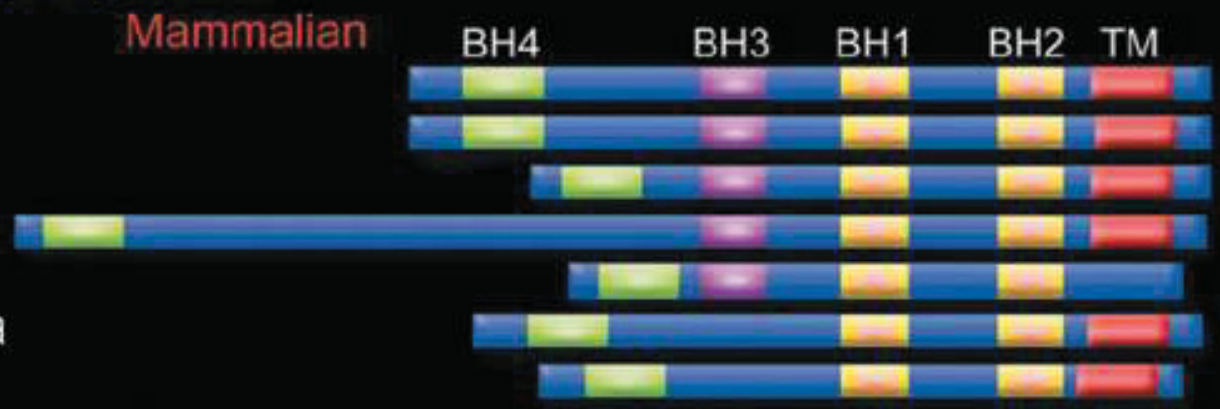

Ced-9

C. elegans

\section{Pro-apoptotic}

Multidomain

Bax

Bak

Bok/Mtd

Mammalian

Bok/nitd

Bid

Bad

Bik/Nbk

Blk

Hrk

Bim/Bod

Bnip3

Nix

Noxa

Puma

Bmf

Egl-1

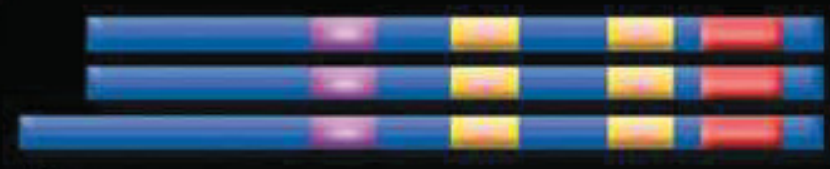

BH3-only

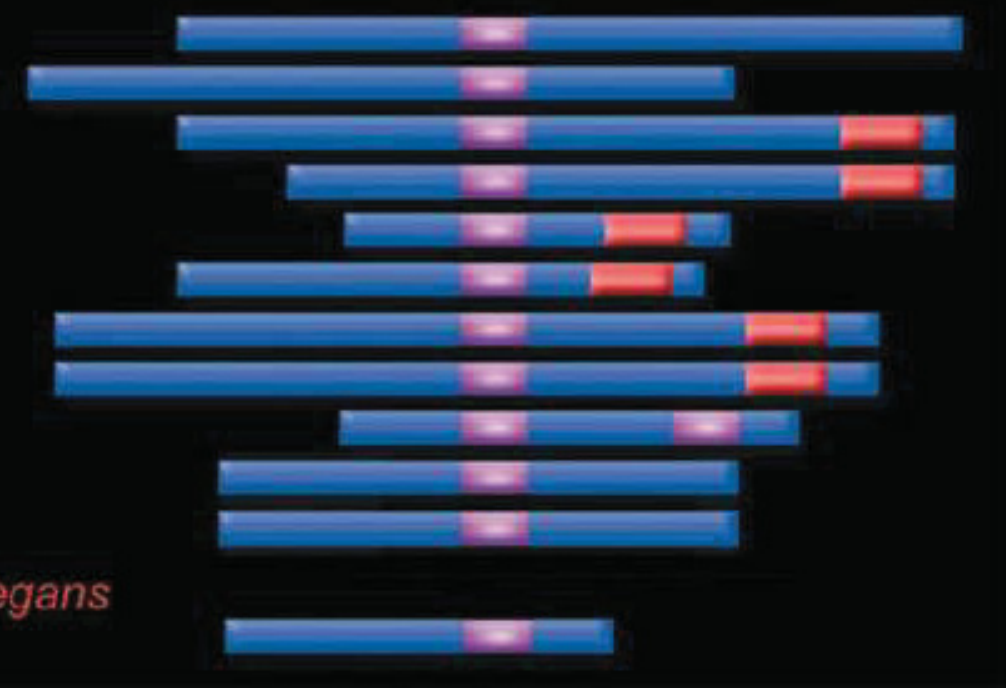




\section{Dual Functions of MCL-1}

MCL-1, the induced myeloid leukemia cell differentiation protein, is an antiapoptotic BCL-2 family member and was initially identified as an immediate-early gene (Myeloid cell leukemia sequence, Mcl-1) expressed during TPA-induced differentiation of ML-1 myeloid leukemia cells (Kozopas et al. 1993). Sequence analysis reveals that the carboxy-terminus of MCL-1 shares homology with other pro-apoptotic BCL-2 family members, containing three putative $\mathrm{BH}$ domains $(\mathrm{BH} 2, \mathrm{BH} 1$ and $\mathrm{BH} 3)$, which confer the ability to interact with other family members, and a hydrophobic transmembrane domain at the extreme carboxy-terminus (Kozopas et al. 1993). However, the amino-terminus of MCL-1 is poorly conserved as it is much longer than other pro-apoptotic BCL-2 family members and is intrinsically unstructured and excluded from structural study (Day et al. 2005). Truncation of either the carboxy- or amino-terminus of MCL-1 does not abolish its ability to bind pro-apoptotic BCL-2 family members (Day et al. 2005).

A variety of growth factors and cytokines have been revealed to regulate MCL-1 expression. Interleukin-7 (IL-7) stimulation could upregulate MCL-1 expression in both mature lymphocytes and immature progenitors (Opferman et al. 2003; Dzhagalov et al. 2008; Dunkle et al. 2011). And the Mcl-1 mRNA level is also induced in hematopoietic stem cells in response to stem cell factor (SCF) and IL-6 (Huang et al. 2000; Opferman et al. 2005). Granulocyte-macropahge colony-stimulating factor (GM-CSF) and IL-3 could enhance MCL-1 expression in cell lines through PI3K/Akt signaling pathway (Wang et al. 1999b). And conversely, IL-3 withdraw in BAF/3 cells leads to a decrease in MCL-1 expression (Maurer et al. 2006). In addition, $M c l-1$ mRNA translation is regulated by mTORC1 pathway (Mills et al. 2008). Therefore, MCL-1 can be regulated at both transcriptional and translational levels.

In addition, post-translational modification can also regulate MCL-1 expression. MCL-1 possesses multiple potential phosphorylation sites, and their modification may have different effects on MCL-1 anti-apoptotic function. For example, cyclin dependent kinase 1 (CDK1), CDK2 and c-Jun $\mathrm{NH}(2)$-terminal kinase (JNK)-mediated phosphorylation of $\mathrm{Ser}^{64}$ enhances the interaction between MCL-1 and pro-apoptotic proteins to facilities its pro-survival function (Kobayashi et al. 2007). Phosphorylation of $\mathrm{Thr}^{92}$ in conjunction with $\mathrm{Thr}^{163}$ by extracellular signal-regulated kinase-1 (ERK-1) stabilizes MCL-1 and promotes survival (Ding et al. 2008). In contrast, phosphorylation of $\mathrm{Ser}^{155}$ in conjunction with $\mathrm{Ser}^{159}$ and $\mathrm{Thr}^{163}$ by glycogen synthase kinase-3 (GSK3) destabilizes MCL-1 and impairs its anti-apoptotic function (Ding et al. 2007).

The amino-terminus of MCL-1 contains two PEST sequences that are typically present in short lived proteins (Kozopas et al. 1993). And MCL-1 does have a short halflife that distinguishes it from other BCL-2 family members (Nijhawan et al. 2003). MCL1 undergoes both ubiquitin-dependent and -independent degradation (Stewart et al. 2010). Three E3 ubiquitin-ligases have been reported to promote the ubiquitinylation of MCL-1, including MCL-1 ubiquitin ligase E3 (MULE), a HECT-domain containing E3 (Zhong et al. 2005), as well as B-TrCP and FBW7, the SKP1-cullin-1-F-box (SCF) complex E3 ligases in a phosphorylation dependent manner (Ding et al. 2007; Inuzuka et al. 2011). 
Moreover, MCL-1 degradation is inhibited by the USP9X deubiquitinase, stabilizing MCL-1 protein expression and leading to apoptotic resistance (Schwickart et al. 2010).

MCL-1 is localized to various intracellular membranes, including the mitochondria (Yang et al. 1995), the endoplasmic reticulum (ER) (Germain and Duronio 2007) and the nuclear envelope (Fujise et al. 2000; Jamil et al. 2005). The predominant mitochondrial localization of MCL-1 is necessary for its anti-apoptotic function, while it has been reported that nuclear localized MCL-1 interacts with CDK1 and proliferating cell nuclear antigen (PCNA) to modulate cell cycle progression.

\section{Anti-apoptotic MCL-1 and Cell Survival}

As an anti-apoptotic protein, the classical function of MCL-1 is to promote survival via interacting with the pro-apoptotic family members. Quantitative assessment of the affinity between pro-survival BCL-2 proteins and the BH3 domains of their proapoptotic partners vary enormously and MCL-1 selectively binds to specific proapoptotic family members (Chen et al. 2005b). While all anti-apoptotic proteins can interact with $\mathrm{BH} 3$-only proteins including BIM, PUMA and tBID, MCL-1 and A1 are engaged in the interaction with NOXA. In contrast to BCL-2, BCL- $\mathrm{X}_{\mathrm{L}}$ and BCL-W, MCL-1 does not interact with BAD (Chen et al. 2005b). Moreover, the multidomian proapoptotic protein BAK can interact with MCL-1 and BCL-X $\mathrm{L}_{\mathrm{L}}$, but not BCL-2 or A1 (Willis et al. 2005). MCL-1 also interacts with BAX (Sedlak et al. 1995) and can protect myeloid progenitors against apoptosis in response to various death stimuli including chemotherapeutic drug, calcium ionophore, UV irradiation, and growth factor withdrawal (Zhou et al. 1997).

The role of anti-apoptotic BCL-2 family members in regulating homeostasis during various stages of hematopoiesis has been revealed via loss of function studies (Figure 1-3). Bcl-2 deficient mice are viable at birth but die within a few months and exhibit renal failure and apoptosis of lymphocytes (Veis et al. 1993). Bcl- $X_{L}$ deficient mice exhibit lethality at embryonic day (E) 13 and excessive death of neurons and immature lymphocytes (Motoyama et al. 1995). $B c l-w$ and $A l$ deficient mice are viable but display defects in spermatogenesis and accelerated neutrophil apoptosis respectively (Hamasaki et al. 1998; Print et al. 1998).

Gene-ablation studies have demonstrated that MCL-1 has distinct roles that are remarkably divergent from other pro-apoptotic BCL-2 family members. Mcl-1 deficient mice exhibit peri-implantation embryonic lethality (E3.5-4) (Rinkenberger et al. 2000). Conditional knockout studies further indicate that MCL-1 is essential for multiple stages of hematopoiesis and lymphopoiesis (Figure 1-3). Conditional deletion of $\mathrm{Mcl}-1$ in the early stage of $\mathrm{T}$ lymphocyte differentiation by using lymphocyte-specific protein tyrosine kinase-Cre (Lck-Cre) results in no viable and mature $M c l-1$-deleted T cell. Deletion of Mcl-1 during early B lymphocyte development by using CD19-Cre increases apoptosis at the pro-B stage. Deletion in mature lymphocytes results in apoptosis, suggesting that 


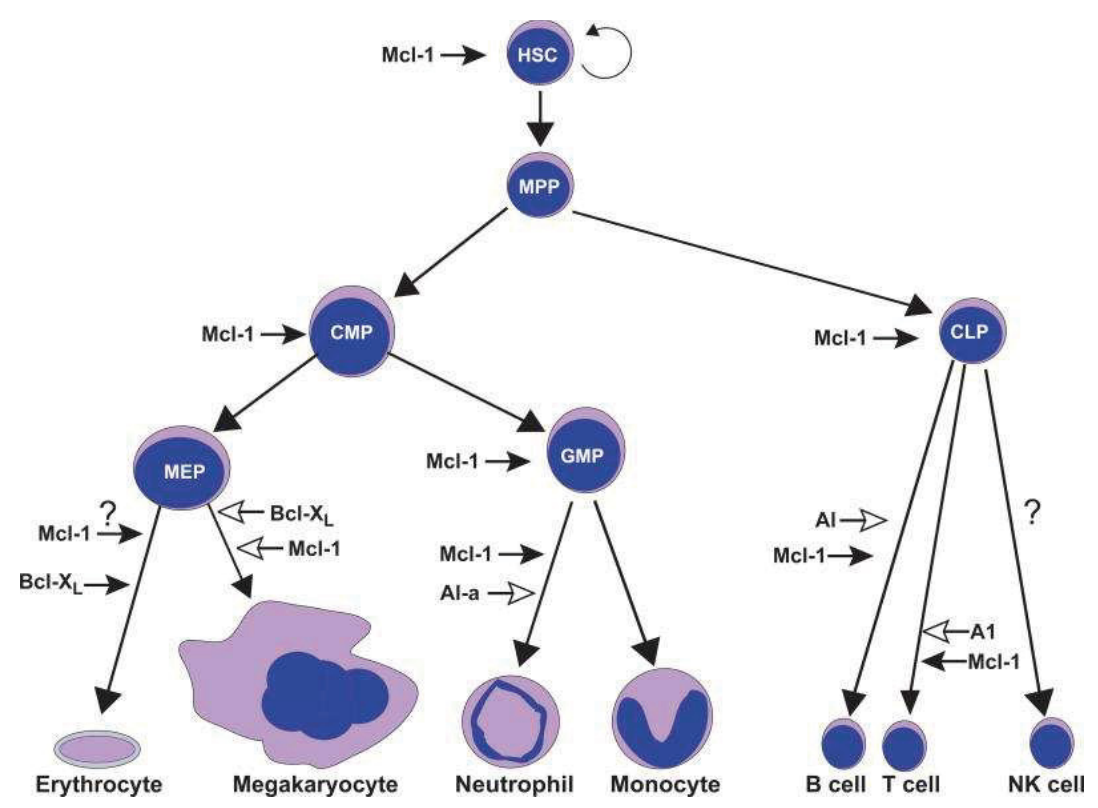

Figure 1-3. Role of anti-apoptotic regulators during hematopoiesis

All blood cell lineages arise from a hematopoietic stem cell (HSC) that is capable of selfrenewal and has an indefinite life-span. HSCs give rise to multi-potent progenitors (MPPs) which still retain the ability to give rise to all blood cell lineages, but lack longterm self-renewal capacity. MPPs can produce two progenitors, common myeloid progenitors (CMPs) and common lymphoid progenitors (CLPs). CLPs can produce the lymphoid lineages (B, T, and perhaps NK cells). CMPs produce at least two other oligopotent progenitor populations, the megakaryocyte erythroid progenitor (MEP) that produce red blood cells (erythrocytes) and megakaryocytes (generates platelets) and the granulocyte monocyte progenitor (GMP) that produces granulocytes (neutrophils) and monocytes (macrophages). Listed beside each differentiation step or progenitor population are the known anti-apoptotic regulators that promote the survival of the given population. Anti-apoptotic MCL-1 has multiple checkpoints as it has been illustrated to be critical for the survival of several multipotent and oligopotent progenitor populations (HSC, CMP, CLP, and GMP) and has been shown to be critical for the differentiation of granulocytes, but interestingly not the monocyte lineage. A1-a, a murine ortholog of BFL-1, and other A1 isoforms have also been shown to play an important role in promoting neutrophil survival in response to stress, but are not absolutely required for development, but primarily effect mature cell survival (indicated by open arrowhead). A1 isoforms also play a role, but are not essential in lymphoid development. In the erythroid lineage, it appears that anti-apoptotic $B C L-X_{L}$ is the essential survival molecule, but unpublished data indicates that MCL-1 may also play an essential role during early differentiation. In the megakaryocytic lineage it appears that while neither MCL-1 nor BCL- $\mathrm{X}_{\mathrm{L}}$ is solely responsible for survival, the two pro-survival molecules appear to have overlapping functions (indicated by open arrowheads) in promoting megakaryocyte survival. Reprinted with permission from Elsevier Ltd: Perciavalle RM, Opferman JT. 2013. Delving deeper: MCL-1's contributions to normal and cancer biology. Trends in cell biology 23: 22-29. 
MCL-1 is required for the development and maintenance of lymphocytes (Opferman et al. 2003).

Furthermore, MCL-1 also plays an indispensable role in early stage of hematopoiesis (Figure 1-3), supported by the observation that conditional deletion of Mcl-1 in early hematopoietic progenitors results in rapid hematopoietic failure. The earliest populations lost are the hematopoietic stem cells (HSCs), common myeloid progenitors (CMPs), and common lymphoid progenitors (CLPs) (Opferman et al. 2005). MCL-1 plays a selective role in myelopoiesis as MCL-1 is required for the development of mature neutrophils from granulocyte precursors. Whereas deletion of $\mathrm{Mcl}-1$ in the myeloid lineage does not block the maturation of macrophages, it is important for survival during macrophage effector function (Steimer et al. 2009). Moreover, MCL-1 is necessary for the survival of nature killer (NK) cells, as specific deletion of $\mathrm{Mcl}-1$ in $\mathrm{NK}$ cells results in absolute loss of NK cells from all tissues due to apoptosis (Sathe et al. 2014).

In addition to its pivotal role at different stages of hematopoiesis, MCL-1 is also required for cortical neurogenesis and the survival of neurons after DNA damage. Conditional deletion of $\mathrm{Mcl}-1$ in the forebrain leads to embryonic lethality at day 15 and deletion of $\mathrm{Mcl}-1$ earlier in the central nerve system leads to lethality at E12.5 (Arbour et al. 2008).

In a human, MCL-1 is expressed in epithelial cells of the skin, gastrointestinal tract, and pulmonary tissues, in cardiac and skeletal muscle, hematopoietic cells, lymphocytes, uterus, adrenal cortex, sympathetic ganglia, pancreatic islets, and hepatocytes (Krajewski et al. 1995). Besides the blood cells, the function of MCL-1 in many other cell types still remains to be established.

\section{MCL-1 and Mitochondrial Physiology}

MCL-1 protein appears as a doublet $(40 \mathrm{kD}$ and $36 \mathrm{kD})$ or sometimes a triplet (40 $\mathrm{kD}, 38 \mathrm{kD}$ and $36 \mathrm{kD}$ ) as detected by immunoblot in many cell types. Several hypotheses have been implied to explain the origin of those bands. Phosphorylation of MCL-1 has been proposed to generate the $40 \mathrm{kD}$ isoform, but phosphatase treatment does not abolish the formation of this band (Perciavalle et al. 2012). Non-canonical translation has been reported to produce the $36 \mathrm{kD}$ species of MCL-1 (Warr and Shore 2008), however, removing the ATG start codon of MCL-1 only produces a $31 \mathrm{kD}$ truncated protein from a downstream ATG codon instead (Perciavalle et al. 2012). Moreover, non-canonical messenger RNA splicing has been proposed to give rise to the $36 \mathrm{kD}$ isoform (Kojima et al. 2010), but ablation of the putative splice donor and acceptor does not abrogate the doublet or triplet formation (Perciavalle et al. 2012). Recently, proteolytic cleavage has been demonstrated to contribute to the generation of different MCL-1 species (Huang and Yang-Yen 2010; Perciavalle et al. 2012). Indeed, amino-terminal Edman sequencing of immunoprecipitated MCL-1 discovered two cleavage sites: the first between MCL-1's isoleucine-10 and glycine-11 and another between leucine-33 and valine-34. As a result, 
proteolysis at the amino-terminus of MCL-1 produces three MCL-1 species with different molecular weight: full-length $(40 \mathrm{kD})$, cleaved at isoleucine-10 $(38 \mathrm{kD})$, and cleaved at leucine-33 (36 kD) (Perciavalle et al. 2012).

Sub-mitochondrial Localization of MCL-1. Further analysis revealed that the various forms of MCL-1 exhibit different mitochondrial sub-localizations; the slowly migrating forms of MCL-1 (40 kD and $38 \mathrm{kD})$ reside on the outer leaflet of the OMM, whereas the $36 \mathrm{kD}$ form of MCL-1 is associated with the inner mitochondrial membrane (IMM) and confined within the mitochondrial matrix in a manner dependent upon the translocases of the outer and inner mitochondrial membrane (TOM and TIM complexes) (Perciavalle et al. 2012). Similarly, another study has also reported that the truncated form of MCL-1 resides in the mitochondrial matrix and the matrix processing peptidase (MPP) is responsible for the cleavage of a mitochondrial targeting sequence (MTS) at the amino-terminus of MCL-1, suggesting that MCL-1 is cleaved during import into the mitochondria (Huang and Yang-Yen 2010).

MCL-1 Promotes Mitochondrial Physiology. The Opferman laboratory has reported that different forms of MCL-1 reside in distinct mitochondrial locations and exhibit separable functions. The OMM localized form of MCL-1 possesses the classical anti-apoptotic activity where it antagonizes BAX and BAK activation to maintain mitochondrial integrity. In contrast, the matrix-localized form of MCL-1 is unable to inhibit apoptosis, but is necessary for maintaining IMM structure, regulating fusion and promoting the assembly of ATP synthase oligomers. As shown in SV40-transformed Mcl- $1^{\text {flox/flox }}$ Rosa-ERCreT2 murine embryonic fibroblast (MEFs), deletion of $M c l-1$ by tamoxifen induction results in decreased ATP production, oxygen consumption, IMM fusion, mitochondrial DNA (mtDNA) level, increased reactive oxygen species (ROS), a loss of electron transport chain (ETC) supercomplex, and a loss of normal mitochondrial cristae structure, which can only be rescued by putting back matrix localized form of MCL-1 (Perciavalle et al. 2012). Thus, besides the classical anti-apoptotic function, MCL-1 is necessary for facilitating mitochondrial homeostasis and supporting mitochondrial bioenergetics function in the model cell line (Figure 1-4).

How MCL-1 promotes mitochondrial physiology and what is the primary deficiency due to its ablation remains elusive. Since cells lacking matrix-localized MCL1 exhibit reduced enzymatic activity of ETC complexes (Perciavalle et al. 2012), it is possible that MCL-1 may directly modulate the function of one or more of the ETC components. As a result, the mitochondrial oxidative phosphorylation (OXPHOS) is diminished and ATP production decreased, leading to the mitochondrial morphological changes, as the morphology of mitochondria is tightly related to the bioenergetic status (Mannella 2006).

Additionally, MCL-1 may regulate IMM structure by facilitating the dimerization and oligomerizaton of $\mathrm{F}_{1} \mathrm{~F}_{0}$-ATP synthase (Perciavalle et al. 2012). ATP synthase is 
(a)

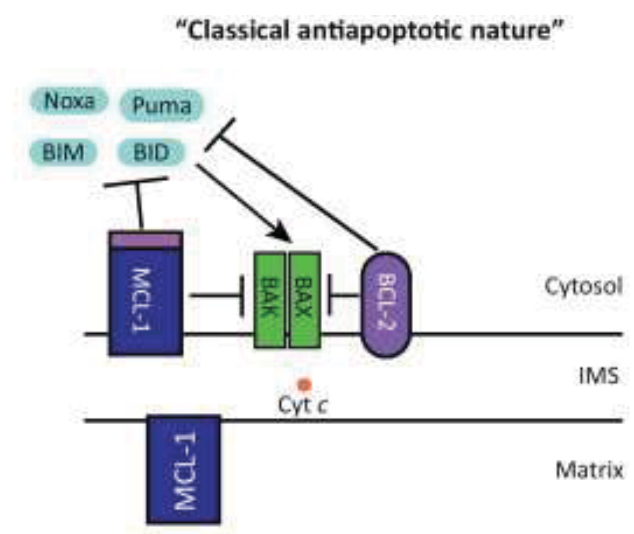

(b) "Role in mitochondrial physiology"
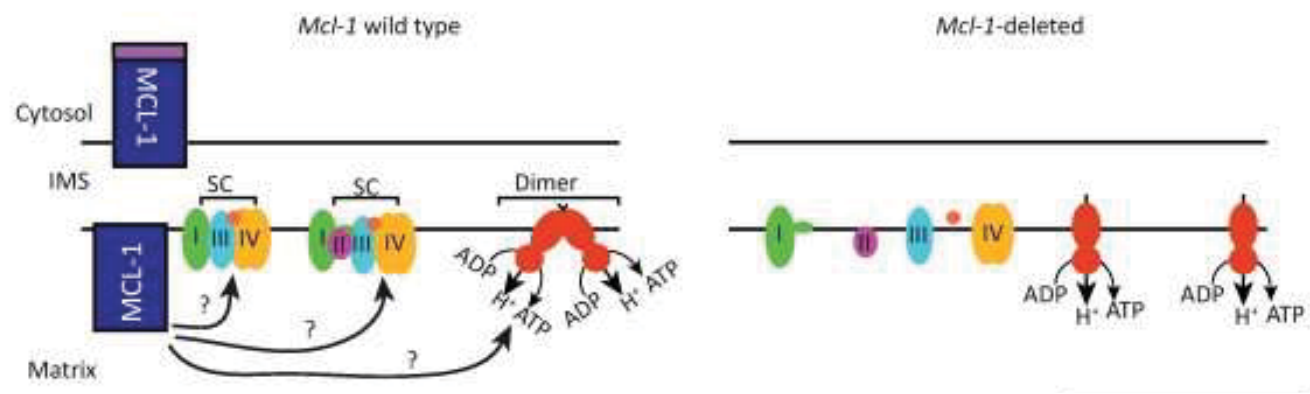

Figure 1-4. Model for MCL-1's potential functions in mitochondria

MCL-1 possesses multiple functions in mitochondria. (a) On the outer mitochondrial membrane (OMM), MCL-1 functions like other antiapoptotic BCL-2 family members where it acts to prevent the activation of BAX and BAK to prevent cell death. MCL-1 can directly bind $\mathrm{BH} 3$-only family members, such as BIM, sequestering them away from the proapoptotic effectors BAX and BAK. Alternatively, MCL-1 may directly bind BAX and BAK and maintain them in an inactive conformation. (b) During mitochondrial importation, the full-length MCL-1 is proteolytically truncated at its amino terminus. The truncated, matrix-localized MCL-1 resides within the inner mitochondrial membrane (IMM), where it functions to maintain mitochondrial cristae ultrastructure and promotes the assembly of the electron transport chain (ETC) complexes into higher-order assemblies known as supercomplexes (SCs). The assembly into supercomplexes has been shown to facilitate electron transport efficiency and reduce the production of deleterious reactive oxygen species. Additionally, matrix-localized MCL-1 facilitates the higherorder assembly of the ATP synthase complexes into dimers and oligomers. Proper assembly of oligomeric ATP synthase has been implicated as being an important determinant of inner membrane cristae structure. Genetic ablation of $\mathrm{Mcl}-1$ results in defects in both supercomplex and ATP synthase oligomer assembly. Whether MCL-1 acts directly or indirectly to facilitate these macromolecular assemblies of the electron transport supercomplexes or ATP synthase oligomers remains unclear. Reprinted with permission from Elsevier Ltd: Perciavalle RM, Opferman JT. 2013. Delving deeper: MCL-1's contributions to normal and cancer biology. Trends in cell biology 23: 22-29. 
composed of a soluble catalytic unit $\left(\mathrm{F}_{1}\right)$ in the mitochondrial matrix, and a membranebound unit $\left(\mathrm{F}_{0}\right)$ anchoring the enzyme in the IMM and mediating the flow of protons through the membrane. The ATP synthase dimers and oligomers are thought to be responsible for the generation of cristae and could facilitate ATP production (Paumard et al. 2002; Strauss et al. 2008).

Deletion of $\mathrm{F}_{0}$ subunit in yeast abolishes dimerization and leads to abnormal cristae structure (Minauro-Sanmiguel et al. 2005). Similarly, deletion of MCL-1 results in diminished ATP synthase dimer and oligomer assembly and disrupted mitochondrial cristae (Perciavalle et al. 2012). Therefore, MCL-1 may act to stabilize the ATP synthase complex, but the mechanism is unknown.

Mitochondria are dynamic organelles constantly undergoing fusion and fission. At steady state, the frequency of fusion and fission events is balanced to maintain the overall normal mitochondrial morphology. Disruption of this balance leads to dramatic change in mitochondrial morphology (Detmer and Chan 2007). Fused mitochondria exhibit elongated, spaghetti-like shape and are extremely interconnected, whereas fissed mitochondria show smaller and fragmented morphology (Detmer and Chan 2007). Mitochondrial fusion enables the exchange of contents between mitochondria, which is pivotal for maintaining mitochondrial homeostasis. Mitochondria with impaired fusion machinery have reduced respiratory capacity, increased ROS production, and induction of mtDNA deletion or mutations (Chen et al. 2005a; Chen et al. 2007; Chen et al. 2010). The mtDNA genome encodes the essential subunits of ETC complexes I, III, IV and V, and therefore is critical for OXPHOS. In the absence of matrix-localized MCL-1, mitochondria exhibit fragmented morphology and less IMM fusion (Perciavalle et al. 2012). Consequently, fusion deficiency could be a possible explanation for decreased levels of mtDNA and subsequent bioenergetic abnormalities observed in $\mathrm{Mcl}$-1-deleted mitochondria.

\section{Cardiomyocyte Death and Heart Disease}

From early embryogenesis through adulthood, the heart is pivotal for all the physiological processes. Thus, it is not surprising that primary and secondary heart diseases can have catastrophic consequences and are the most costly and common causes of adult death worldwide (NHLBI 2012). Insults including ischemia, hypoxia, toxins and drugs can cause heart diseases, which are associated with loss or dysfunction of cardiomyocytes, diminished pump function, abnormal conduction, eventual heart failure and death.

Adult cardiomyocytes are limited in their ability to re-enter the cell cycle, and thus have limited capacity for self-renewal. In mice, when the ventricle apex of the heart is amputated at postnatal day $1(\mathrm{P} 1)$, the heart can undergo full regeneration without scar formation. However, this regenerative ability of neonatal heart is lost after P7 and extensive cardiac fibrosis is observed (Porrello et al. 2011). Although it is reported that in young adult mice under normal homeostatic conditions, cardiomyocyte renewal from pre- 
existing myocytes can occurs at a low rate $(0.76 \%$ per year and decline at age), and increases upon injury, this limited capacity of renewal is still insufficient for regeneration in response to various insults (Senyo et al. 2013). Thus, the heart is particularly sensitive to cardiomyocyte death and exploring how cell death is regulated is critical to developing potential strategies for preventing cardiac damage and preserving cardiac function.

Cardiomyocyte death is a hallmark in the progress of various heart diseases, in which cells die primarily through apoptosis and/or necrosis, and autophagy, which has been associated with cell death as well (Whelan et al. 2010).

\section{Apoptosis and Heart Disease}

Atherosclerotic vascular disease is the major cause of myocardial infarction and heart failure and the disruption of the atherosclerotic plaque is the leading factor of acute coronary syndrome. It is reported that apoptotic macrophages and smooth muscle cells in the plaque can lead to the accumulation of lipid-rich core to facilitate the disruption of plaque and thrombosis (Kockx et al. 1998; Clarke et al. 2008). Reperfusion is the treatment for myocardial infarction, but the reintroduction of oxygen to the infarct area can lead to reperfusion injury. The presence of apoptotic cardiomyocytes has been identified as a significant component of cell loss during reperfusion and contributes to the development of cardiac remodeling and heart failure after myocardial infarction (Gottlieb et al. 1994; Saraste et al. 1997; Levine and Klionsky 2004).

Moreover, increased levels of apoptotic cardiomyocytes have been observed in the hearts of diabetic patients and are implicated in the development of diabetic cardiomyopathy (Cai et al. 2002; Li et al. 2007). Hyperglycemia appears to play a critical role in the pathogenesis of diabetic cardiomyopathy by the induction of cardiomyocyte apoptosis via increased level of ROS and nitrogen species (Cai and Kang 2001). Studies have shown that antioxidants and apoptotic inhibitors can significantly prevent the development of cardiomyopathy suggesting that oxidative stress-mediated apoptosis plays an important role in this process (Ye et al. 2003; Song et al. 2005).

The gp130 is a common subunit of cytokine receptor for the interleukin- 6 family and has been shown to promote cell survival under apoptotic stress in vitro. The cardiacspecific gp130 knockout mice reveal grossly normal phenotype, but develop significant cardiomyocyte apoptosis $(\approx 34 \%$ ) and mostly die within weeks after being exposed to an acute pressure load by surgical constriction of the transverse aorta (Hirota et al. 1999). On the other hand, the overexpression of heterotrimeric $G$ proteins, such as $G_{q \alpha}$ or $G_{s \alpha}$ in the cardiomyocytes may promote apoptosis under stress. Pregnant transgenic mice with $\mathrm{G}_{\mathrm{q} \alpha}$ overexpressed in the heart develop lethal dilated cardiomyopathy within one week after delivery and TUNEL staining reveals a markedly increased level of cardiomyocyte apoptosis $\left(\approx 26 \%\right.$ ) (Adams et al. 1998). Overexpression of $\mathrm{G}_{\mathrm{s} \alpha}$ in the heart also results in increased apoptosis and leads to cardiomyopathy and blocking the $\beta$-adrenergic receptor could prevent cardiac damage, decrease cardiac apoptosis and preserve cardiac function (Asai et al. 1999; Geng et al. 1999). 
Chronic heart failure is the end-stage of various heart diseases and apoptosis has also been revealed to play an important role in the progression of this disease. It is reported that patients with chronic heart failure have higher level of cardiomyocyte apoptosis than normal subjects (0.08-0.25 vs. 0.001-0.002\%) (Saraste et al. 1997; Guerra et al. 1999; Levine and Klionsky 2004). Moreover, genetic and pharmacological studies on animal models suggest a role for cardiomyocyte apoptosis in the progression of chronic heart failure. Kitsis et al. generated transgenic mice overexpressing cardiacspecific ligand-activatable Caspase-8, which is a fusion protein consisting of a FK506 binding domain and the Caspase- 8 catalytic domain. Transgenic mice expressing this fusion protein reveal no obvious observable phenotype after birth, but administration of the dimerizer FK1012, which activates Caspase-8 and in turn Caspase-3, leads to overwhelming cardiomyocyte apoptosis and not surprisingly, rapid death of the animal. However, even in the absence of FK1012, transgenic mice with high expression of the fusion protein still exhibit a low level of spontaneous apoptosis (lower than $0.1 \%$ whereas wild type mice is less than $0.002 \%$ ) in the heart, leading to a severe dilated cardiomyopathy over 8 weeks and death within 2-6 months after birth. Prevention of cardiomyocyte apoptosis with a caspase inhibitor can ameliorate the cardiac dilation and improve cardiac function (Wencker 2003). This suggests that even a very low level of apoptosis can contribute to the pathogenesis of dilated cardiomyopathy and heart failure.

The major signaling pathways involved in the cardiomyocyte apoptosis include the intrinsic apoptosis pathway (mitochondrial death pathway), extrinsic apoptosis pathway (death receptor pathway), and endoplasmic reticulum (ER)-stress death pathway.

The Intrinsic Apoptosis Pathway and Heart Disease. Just like other cell types, mitochondria play a central role in the initiation and regulation of the intrinsic apoptosis pathway in cardiomyocytes. In response to various stresses including nutrient deprivation, oxidative stress and DNA damage, mitochondria release several apoptogenic proteins such as cytochrome $c$, apoptosis inducing factor (AIF), endonuclease G (Endo G), second mitochondria-derived activator of caspases (Smac/Diablo), and HtrA2/Omi to induce apoptosis. This pathway appears to play a significant role particularly in ischemia/reperfusion injury and heart failure (Chen et al. 2002; Scarabelli et al. 2002).

Abundant evidence demonstrates that BCL-2 family members also tightly regulate the intrinsic apoptosis pathway in the myocardium. And the balance between pro- and anti-apoptotic BCL-2 proteins can determine the outcome of pathological processes in the heart (Latif et al. 2000; Baldi et al. 2002; Di Napoli et al. 2003).

Anti-apoptotic BCL-2 Family Members. Anti-apoptotic BCL-2 family members have been shown to protect cardiomyocytes from various stresses and considered to have therapeutic potential in several cardiac disease models. For example, BCL-2 can prevent p53-mediated cardiomyocyte apoptosis (Kirshenbaum and de Moissac 1997). Transgenic mice with BCL-2 overexpressed in the heart have fewer apoptotic cells, reduced infarct size, improved cardiac function and prolonged survival time after ischemia/reperfusion 
injury (Brocheriou et al. 2000; Chen et al. 2001; Imahashi et al. 2004). Desmin knockout mice develop cardiomyopathy characterized by extensive fiber loss, mitochondrial damage and later heart failure (Milner et al. 1996; Milner et al. 1999). And overexpression BCL-2 in the heart can attenuate the cardiomyopathy phenotype by blocking apoptotic cell death and preventing mitochondrial defects (Milner et al. 2000; Weisleder et al. 2004; Maloyan et al. 2010). In addition, overexpression of BCL- $\mathrm{X}_{\mathrm{L}}$ in H9c2 cardiac cells inhibits doxorubincin and hypoxia-induced apoptosis (Reeve et al. 2007). Transgenic mice with increased accumulation of mtDNA mutation reveal a strong pro-survival response by upregulating BCL-2 and BCL- $\mathrm{X}_{\mathrm{L}}$ (Zhang et al. 2005).

Moreover, other protective functions of BCL-2 have also been identified. One mechanism for ischemic damage is the inhibition of mitochondrial ATP production due to lack of oxygen, which induces $\mathrm{F}_{1} \mathrm{~F}_{0}$-ATPase running inversely to consume glycolytic ATP, leads to energy depravation (Rouslin et al. 1986; Vuorinen et al. 1995). Furthermore, overexpression of BCL-2 in the heart decreases the rate of ATP decline and reduces acidification, suggesting that BCL-2 may protect myocardium during ischemia by inhibiting consumption of glycolytic ATP generated by inversed $\mathrm{F}_{1} \mathrm{~F}_{0}$-ATPase (Imahashi et al. 2004). Additionally, during ischemia, the sodium calcium exchanger (NCX) extrudes $\mathrm{Na}^{+}$and the cells become overloaded with $\mathrm{Ca}^{2+}$ (Karmazyn and Moffat 1993). BCL-2 has been shown to reduce the NCX activity and increase resistance to mitochondrial permeability transition by increasing the $\mathrm{Ca}^{2+}$ threshold for the opening of mitochondrial permeability transition pore (mPTP) in the heart (Zhu et al. 2001). These studies indicate that anti-apoptotic BCL-2 proteins can protect cardiomyocytes from cell death through multiple mechanisms and have promising therapeutic potentials to prevent and treat heart diseases.

Pro-apoptotic BCL-2 Family Members. The pro-apoptotic BCL-2 proteins have been implicated in the pathogenesis of various heart diseases. The pro-apoptotic BH3only proteins function as stress sensors and integrate cell death stimuli. Several BH3-only proteins including BID, BAD, PUMA, BNIP3 and NIX/BNIP3L, have been implicated in cardiomyocyte death. BID is cleaved during myocardial ischemia/reperfusion, resulting in the release of cytochrome $c$ to induce apoptosis. BAD is significantly induced during ischemia/reperfusion accompanied by a reduction in BCL-2 and BCL- $\mathrm{X}_{\mathrm{L}}$ (Murriel et al. 2004). Puma is induced in cardiomyocytes upon hypoxia-reoxygenation, and deletion of Puma decreases infarct size and improves cardiac function after ischemia/reperfusion (Toth et al. 2006). BNIP3 and NIX/BNIP3L are two BH3-only proteins that have been extensively studied in the heart. These two proteins are upregulated in response to various stresses by different pathways: BNIP3 by hypoxia via hypoxia induced factor-1 $\alpha$ (HIF-1 $\alpha)$ and E2F-1 (Bruick 2000; Kubasiak et al. 2002; Regula et al. 2002; Galvez et al. 2006; Yurkova et al. 2008), and NIX by $\mathrm{G}_{\mathrm{q}} \alpha$-mediated stimuli, such as phenylephrine (Galvez et al. 2006). BNIP3 is activated by oxidative stress during myocardial reperfusion and then activates downstream BAX and BAK to induce apoptosis (Kubli et al. 2008). In addition, NIX/BNIP3L has also been associated with cardiac hypertrophy and development of cardiomyopathy (Diwan et al. 2008) 
Activation of $\mathrm{BH} 3$-only proteins in response to stress induces the activation of $\mathrm{BAX}$ and BAK. Activated BAX and BAK undergo oligomerization and induce the permeabilization of the outer mitochondrial membrane (OMM) allowing the release of apoptogenic proteins from the intermembrane space. Therefore BAX and BAK are essential for the mitochondrial death pathway. BAX is activated in cardiomyocyte in response to oxidative stress (Gustafsson et al. 2004). During ischemia, BAX is translocated to mitochondria and this is shown to be dependent on AMPK and p38 MAPK in neonatal cardiomyocytes (Capano and Crompton 2006). Moreover, BAX deficient mice revealed less mitochondrial damage and reduced infarct size after ischemia/reperfusion injury (Hochhauser et al. 2003), indicating that BAX is important in this process.

The Extrinsic Apoptosis Pathway and Heart Disease. Several studies have shown that the extrinsic apoptosis pathway contributes to the pathogenesis of heart diseases. Both death receptors Fas and tumor necrosis factor receptor (TNFR1) are presented in cardiomyocytes. It is reported that the ligand of TNFR1, TNF- $\alpha$, is abundant in cardiomyocytes (Giroir et al. 1992; Kapadia et al. 1995), and its expression in failing human myocardium is higher than non-failing heart (Doyama et al. 1996; Torre-Amione et al. 1996). Patients with end-stage congestive heart failure have elevated circulating TNF- $\alpha$, and its level is related to the severity of the disease (Levine et al. 1990; Dutka et al. 1993; Ferrari et al. 1995). In addition, the level of TNF- $\alpha$ in both the heart and circulation is increased during ischemia/reperfusion (Jeremias et al. 2000; Gilles et al. 2003). Transgenic mice with TNF- $\alpha$ overexpressed in the heart developed dilated cardiomyopathy and heart failure (Kubota et al. 1997; Bryant et al. 1998), suggesting that the TNF- $\alpha$-mediated death receptor pathway is detrimental to the heart.

Activation of the Fas induced death receptor pathway has been observed in cardiomyocytes, and stress such as hypoxia can induce the expression of both Fas and FasL in the cultured cardiomyocytes (Tanaka et al. 1994). There is evidence indicating that Fas-mediated apoptosis contributes to myocardial ischemia/reperfusion injury and post-infarction remodeling. Studies have shown that mice lacking functional Fas ( $\mathrm{lpr}$ mice) are resistant to ischemia/reperfusion injury and have reduced infarct size and apoptosis compared to control mice (Jeremias et al. 2000; Lee et al. 2003). During myocardial infarction, granulation tissue cells are cleared by apoptosis to complete scar formation. Blocking apoptosis could improve post-infarction remodeling and heart failure. Both $l p r$ mice and Fas ligand deficient mice ( $g l d$ mice) revealed significantly fewer apoptotic granulation tissue cells compared to control mice, and ventricular remodeling and heart failure is attenuated. Mice transfected with adenovirus encoded a competitive FasL inhibitor (sFas) on the third day of infarction have alleviated heart failure symptoms and prolonged survival ( $\mathrm{Li}$ et al. 2004). Therefore, Fas-mediated apoptosis plays a role in the progression of heart diseases. 
The ER-stress Death Pathway and Heart Disease. The endoplasmic reticulum (ER) is essential for multiple cellular processes, including intracellular calcium homeostasis, protein secretion and lipid biosynthesis, which are required for cell survival and normal functions. Many insults, including oxidative stress, glucose deprivation and aberrant $\mathrm{Ca} 2+$ regulation cause the accumulation of unfolded proteins in the ER, triggering the unfolded protein response (UPR) to restore ER homeostasis (Szegezdi et al. 2006b). The UPR activates transcriptional programs to enhance the protein folding capacity of the ER, promote the degradation of misfolded proteins and inhibit protein synthesis to reduce the influx of new proteins into the ER. Prolonged and excessive ER stress causes failure of the UPR and induces cell death typically in the form of apoptosis (Xu et al. 2005).

Many mechanisms have been hypothesized to explain the ER stress-mediated apoptosis. It is reported that Caspase-12 is activated upon ER stress, and activated Caspase-12 activates Caspase-3 to induce apoptosis (Szegezdi et al. 2006b). ER stress also induces the expression of transcriptional factor $\mathrm{C} / \mathrm{EBP}$ homologous protein (CHOP/GADD 153) and p53, increasing the expression of pro-apoptotic proteins such as PUMA and NOXA (Li et al. 2006).

Several studies have demonstrated the contribution of ER stress to heart diseases. For instance, pharmacological ER stress inducer induces apoptosis in cultured cardiomyocytes, and pressure overload inducing extensive ER stress leads to cardiac hypertrophy and heart failure in mice through the CHOP-dependent pathway (Okada et al. 2004). ER stress is also enhanced during myocardial ischemia/reperfusion injury through upregulation of CHOP and activation of Caspase-12, and participates in the development of ischemic heart disease (Azfer et al. 2006; Szegezdi et al. 2006a; Thuerauf et al. 2006). Evidence also indicates that ER stress mediated apoptosis plays a role in the pathogenesis of diabetic cardiomyopathy ( $\mathrm{Li}$ et al. 2007). Moreover, transgenic mice with mutated KDEL receptor (a receptor for ER chaperones) develop dilated cardiomyopathy (Hamada et al. 2004).

\section{Necrosis and Heart Disease}

Cardiomyocyte necrosis has been well described in heart ischemia/reperfusion injury and heart failure. Generally, initiators of necrosis include calcium overload, hypoxia and ROS (Nakayama et al. 2007). Mitochondrial permeability transition pore (mPTP) is considered to be the executor in this event. The mPTP is generally hypothesized to consist of the voltage-dependent anion channel (VDAC) on the OMM, the adenine nucleotide translocator (ANT) in the IMM, and Cyclophilin-D in the matrix, which is still under debate, and only Cyclophilin-D has been shown to be an essential component of the mPTP by genetic ablation (Kroemer et al. 2007).

Cardiomyocytes lacking Cyclophilin-D are resistant to calcium overload and ROS damage induced cell death, but still sensitive to apoptotic stimuli. Cyclophilin-D knockout mice exhibit reduced infarct size after ischemia/reperfusion injury, and 
overexpression of Cyclophilin-D was able to restore the death response (Baines et al. 2005; Nakagawa et al. 2005). These studies indicate the importance of Cyclophilin-D mediated necrosis in the process of ischemia/reperfusion. In addition, calcium overload in transgenic mice results in cardiomyocytes necrosis and heart failure, and the phenotype can be rescued by deletion of Cyclophilin- $D$, suggesting that necrosis mediates heart failure (Nakayama et al. 2007). Furthermore, Cyclophilin-D has been shown to be involved in the pathogenesis of muscular dystrophy. Genetic ablation and pharmacological inhibition of Cyclophilin-D could markedly alleviate the muscular phenotype by reducing myofiber necrosis (Millay et al. 2008; Tiepolo et al. 2009).

More recently, the pro-apoptotic protein BAX is reported to play a role in necrosis. Co-deletion of Bax and Bak significantly reduce the infarct size after myocardial infraction, however, further deletion of Cyclophilin-D over the Bax and Bak-deficient background fails to show additional reduction in infarct size. Bax and Bak-deleted mitochondria and cells are resistant to MPTP opening and necrosis. Reconstitution with wild-type BAX or an oligomerization-deficient mutant that cannot support apoptosis restores the opening of $\mathrm{mPTP}$ and necrosis. Furthermore, induction of mitochondrial fusion potentiates $\mathrm{MPTP}$ opening in the absence of BAX and BAK, indicating that deletion of Bax and Bak may also down-regulate necrotic cell death by increasing the threshold for the opening of the mPTP (Whelan et al. 2012). Thus, BAX and BAK contribute to the pathogenesis of myocardial infarction not only through apoptosis, but also through necrosis.

\section{Autophagy-associated Cell Death and Heart Disease}

In contrast to apoptosis and necrosis, autophagy is primarily a survival mechanism. It is a conserved cellular process for degradation and recycling of cytoplasmic components, such as long-lived proteins and large organelles. Autophagy provides amino acids and fatty acids to cells under nutrient deprivation. However, the role of autophagy in cell death is still under debate and seems to be circumstantial and not yet defined at the mechanistic level. In the heart, the role of autophagy has been reported to be protective as well as deleterious to cardiac function (Nishida et al. 2008).

Induction of autophagy is observed during myocardial infarction and ischemia/reperfusion injury and plays distinct roles during ischemia and reperfusion. Energy deprivation in infarction and ischemia activates 5'-adenosine monophosphateactivated protein kinase (AMPK) to induce autophagy. Transgenic mice with overexpression of a dominant-negative AMPK reveal autophagy inhibition and enlarged infarct size, suggesting that autophagy promotes survival under this condition (Takagi et al. 2007). In contrast, during reperfusion, the enhancement of autophagy is mediated by Beclin-1 induction rather than AMPK activation, which is detrimental to the heart, as significantly less autophagy and attenuated cardiac injury is observed in Beclin- ${ }^{+/-}$mice (Matsui et al. 2007). 
Failing human hearts have increased amount of autophagosomes, indicating the induction of autophagy (Hein et al. 2003; Kostin et al. 2003). The consequences of autophagy enhancement in heart failure have been addressed by several studies. Cardiacspecific deletion of Atg-5 during adulthood leads to cardiac hypertrophy, left ventricular dilation, loss of cardiac contractility, abnormal sarcomere and mitochondrial structure, accumulation of ubiquitinylated protein and cardiomyocyte apoptosis. Deletion of Atg-5 at embryonic day (E) 7.5-8 exhibits no cardiac phenotype due to presumable compensation. However, hemodynamic overload or $\beta$-adrenergic stimulation leads to cardiac dysfunction, left ventricle dilation, and fatal heart failure thereafter (Nakai et al. 2007). This study indicates that autophagy is protective for heart under basal conditions and induction of autophagy is a compensatory mechanism for heart failure. In another study, heterozygous disruption of Beclin-1, a protein required for early autophagosome formation, decreases autophagy, attenuates severe pressure load-induced cardiac remodeling and heart failure. Conversely, overexpression of Beclin-1 enhances autophagy and accentuates pathological remodeling (Zhu et al. 2007). This study concludes that autophagy plays a role in the pathogenesis of pressure overload-induced heart failure, which is controversial to the first study. Moreover, Beclin- $I^{+/-}$has been used to access the role of autophagy in Desmin-related cardiomyopathy. Heterozygous disruption of Beclin-1 leads to decreased autophagy, accumulation of ubiquitinylated proteins, fibrosis, heart failure and increased mortality (Tannous et al. 2008). This study concludes that autophagy plays a protective role in Desmin-related cardiomyopathy. Thus, further studies need be done to clarify the different roles of autophagy in heart failure.

\section{Mitochondria and Heart Disease}

Mitochondria are cytoplasmic double-membrane organelles, which have long been known to play a pivotal role in the life of the cell. They carry out the energyyielding OXPHOS that provides the most of ATP necessary for all cellular functions. Interruption of mitochondria function, for example by cyanide poisoning, rapidly causes death in vivo (Newmeyer and Ferguson-Miller 2003). Mitochondria OXPHOS generates ATP and water via oxidation of hydrogen from carbohydrates and fats. Hydrogen from carbohydrates is recovered through the tricarboxylic acid (TCA) cycle, while hydrogen from fats is recovered through fatty acid $\beta$-oxidation. Electrons from hydrogen are either stripped to nicotinamide adenine dinucleotide $\left(\mathrm{NAD}^{+}\right)$to produce $\mathrm{NADH}+\mathrm{H}^{+}$and then transferred to electron transport chain (ETC) complex I (NADH dehydrogenase), or to flavin adenine dinucleotide (FAD) to generate FADH2 and then transferred to complex II (succinate dehydrogenase, SDH). The electrons are sequentially passed to ubiquinone (coenzyme Q, CoQ) to give ubisemiquinone and ubiquinol. Ubiquinol transfers the electrons to complex III (ubiquinol:cytochrome- $c$ oxidoreductase), cytochrome- $c$, complex IV (cytochrome- $c$ oxidase, COX) and finally to oxygen to give $\mathrm{H}_{2} \mathrm{O}$. The energy released by the ETC is used to pump protons out across the IMM to generate the proton electrochemical gradient. The potential energy stored in the proton gradient is used to condense ADP + Pi to ATP via complex V (ATP synthase), driven by the flow of protons from the intermembrane space back into the mitochondrial matrix through the complex $\mathrm{V}$ 
proton channel. Matrix ATP is then exchanged for cytosolic ADP by the IMM ANTs (Goldstein et al. 1999; Wallace 2002; Wallace and Fan 2009) (Figure 1-5).

Mitochondrial OXPHOS also generates the $\operatorname{ROS}\left(\mathrm{O}_{2}{ }^{-}, \mathrm{H}_{2} \mathrm{O}_{2}\right.$, and $\left.\mathrm{OH}^{\circ}\right)$ as byproducts. When ETC is inhibited, the electrons from complex I can be transferred to the $\mathrm{O}_{2}$ on the matrix side of the IMM or from complex III to $\mathrm{O}_{2}$ on the cytosolic side of the IMM to generate superoxide anion $\left(\mathrm{O}_{2}{ }^{-}\right) \cdot \mathrm{O}_{2}{ }^{-{ }^{-}}$then is further converted to hydrogen peroxide $\left(\mathrm{H}_{2} \mathrm{O}_{2}\right)$ by either matrix-specific manganese superoxide dismutase (MnSOD) encoded by nuclear DNA (nDNA) Sod 2 gene or IMS and cytosolic $\mathrm{Cu} / \mathrm{ZnSOD}$ encoded by $\mathrm{Sod} 1$ gene. $\mathrm{H}_{2} \mathrm{O}_{2}$ is converted to $\mathrm{H}_{2} \mathrm{O}$ by glutathione peroxidase $(\mathrm{GPx}) . \mathrm{H}_{2} \mathrm{O}_{2}$ can also be converted to the highly reactive hydroxyl radical $\left(\mathrm{OH}^{\circ}\right)$ in the presence of reduced transition metals, which is the most potent oxidizing agent among the ROS (Figure 1-5). ROS are toxic byproducts for cells. Acute ROS exposure can inactivate the iron-sulfur (Fe-S) center of ETC complex I, II, III, and TCA aconitase, blocking the energy production of mitochondria. Chronic ROS exposure can lead to oxidative damage to mitochondrial and cellular DNA, proteins and lipids, and finally affect a variety of cellular processes (Wallace 1999; Wallace 2002; Wallace and Fan 2009).

In addition to being the "powerhouse" and endogenous ROS resource of the cell, mitochondria are also involved in many other processes, such as cell death (discussed intensively in previous section), development, cell cycle, and cellular signaling (McBride et al. 2006). Therefore, mitochondria have been implicated in many human diseases, which have been recapitulated in several mouse models. It is now clear that mitochondrial dysfunction can cause an assemblage of clinical problems and commonly present in tissues with high-energy requirements, such as heart, muscle, liver, kidney, nervous system and endocrine system (Wallace 1999; Wallace 2002).

\section{Mitochondria in the Heart}

As the hardest working organ in body, the heart produces and utilizes approximately $30 \mathrm{~kg}$ of ATP every day to keep basal metabolism and normal heart contraction for sustaining systemic and pulmonary blood pressure (Ferrari 2003). ATP is the primary source of energy for the physiological functions of the heart, including contraction, relaxation, electrical excitation and recovery of the resting electrochemical gradients across membranes. Under physiological conditions, 90\% of heart's ATP is produced in cardiomyocyte mitochondria by oxidative phosphorylation (Das 1991). Substrate oxidation in heart changes during organ development. The fetal and newborn heart uses glucose and lactate as the main sources of energy, whereas the postnatal heart preferentially oxidizes fatty acids and produces energy sequentially through $\beta$-oxidation, Kreb's cycle and the respiratory chain (Carvajal and Moreno-Sanchez 2003). Ultrastructural studies have revealed that cardiac muscle is packed with mitochondria, accounting for $\sim 35 \%$ of myocardial mass (Sommer and Waugh 1978), making the myocardium one of the most mitochondria-rich tissues. In normal myocardium, mitochondrial are usually tightly aligned between myofibrils, but are also found beneath 


\section{Figure 1-5 Mitochondria and cancer}

Mitochondria lie at the nexus of most biosynthetic pathways, produce much of the cellular energy through oxidative phosphorylation (OXPHOS), regulate mitochondrial and cellular redox status, generate most of the reactive oxygen species (ROS), regulate $\mathrm{Ca} 2+$ concentrations and can initiate apoptosis by the activation of the mitochondrial permeability transition pore (mtPTP). The mtPTP can be activated by a decreased membrane potential, high-energy phosphates (such as ADP), a more-oxidized redox status, and/or increased mitochondrial matrix $\mathrm{Ca} 2+$ and ROS concentrations. Reducing equivalents and acetyl-CoA enter the mitochondrion via pyruvate and fatty acids. Pyruvate is transported through the mitochondrial inner membrane by the pyruvate transporter (PT)118, binds to pyruvate dehydrogenase (PDH), which may be membraneassociated, and is oxidatively decarboxylated to produce acetyl-CoA. Inhibition of mitochondrial function results in pyruvate accumulation in the cytosol, where it can be reduced to lactate. Fatty acids are imported into the mitochondrion bound to carnitine. In the cytosol, fatty acids bound to CoA are transferred to carnitine, transported through the outer and inner mitochondrial membranes, and then transferred back to CoA for $\beta$ oxidation. The transfer of fatty acyl groups between CoA and carnitine is mediated by the carnitine palmitoyltransferases (not shown). As a by-product of OXPHOS - the substrates and products of which are transported through the outer membrane by the voltage-dependent anion channels (VDACs) - mitochondria generate ROS by the donation of excess electrons from complexes I and III directly to $\mathrm{O} 2$ to generate superoxide anions $(\mathrm{O} 2 \bullet-)$. Matrix $\mathrm{O} 2 \bullet-$, primarily from complex I, is dismutated to $\mathrm{H} 2 \mathrm{O} 2$ by the mitochondrial matrix Mn superoxide dismutase (MnSOD; also known as SOD2), while intermembrane-space $\mathrm{O} 2 \bullet-$, which is primarily from complex III, is dismutated by $\mathrm{Cu} / \mathrm{Zn}$ superoxide dismutase (Cu/ZnSOD; also known as SOD1). H2O2 can be reduced to water by glutathione peroxidase using reduced glutathione as an electron donor. Oxidized glutathione is reduced by glutathione reductase using NADPH as a reductant. In the presence of reduced transition metals, $\mathrm{H} 2 \mathrm{O} 2$ can be reduced to hydroxyl radicals $(\bullet \mathrm{OH})$, which are the most reactive ROS. The mtPTP is a protein complex that is thought to include the translocator protein (TSPO; also known as PBR), an unknown inner-membrane channel, adenine nucleotide translocators (ANTs) and the cyclosporine-A-sensitive cyclophilin D (CYPD; also known as PPID), which are regulatory, in association with the BCL-2 pro- and anti-apoptotic family members. When activated, the mtPTP forms a channel between the inner and outer membranes, which short-circuits $\triangle \mathrm{P}$. This is associated with the aggregation of BAX and BAD in the mitochondrial outer membrane to form a megachannel. The megachannel releases proapoptotic proteins from the intermembrane space into the cytosol to initiate the degradation of the cellular proteins and DNA. AIF, apoptosis-inducing factor; CoA-SH, coenzyme A with a free sulphydryl group; CoQ, coenzyme Q10; ENDOG, mitochondrial endonuclease G; GPX, glutathione peroxidase; LDH, lactate dehydrogenase; OAA, oxaloacetate; SMAC, second mitochondria-derived activator of caspase; TCA, tricarboxylic acid. Reprinted with permission from Macmillan Publishers Ltd: Wallace DC. 2012. Mitochondria and cancer. Nature Reviews Cancer 12: 685-698. 


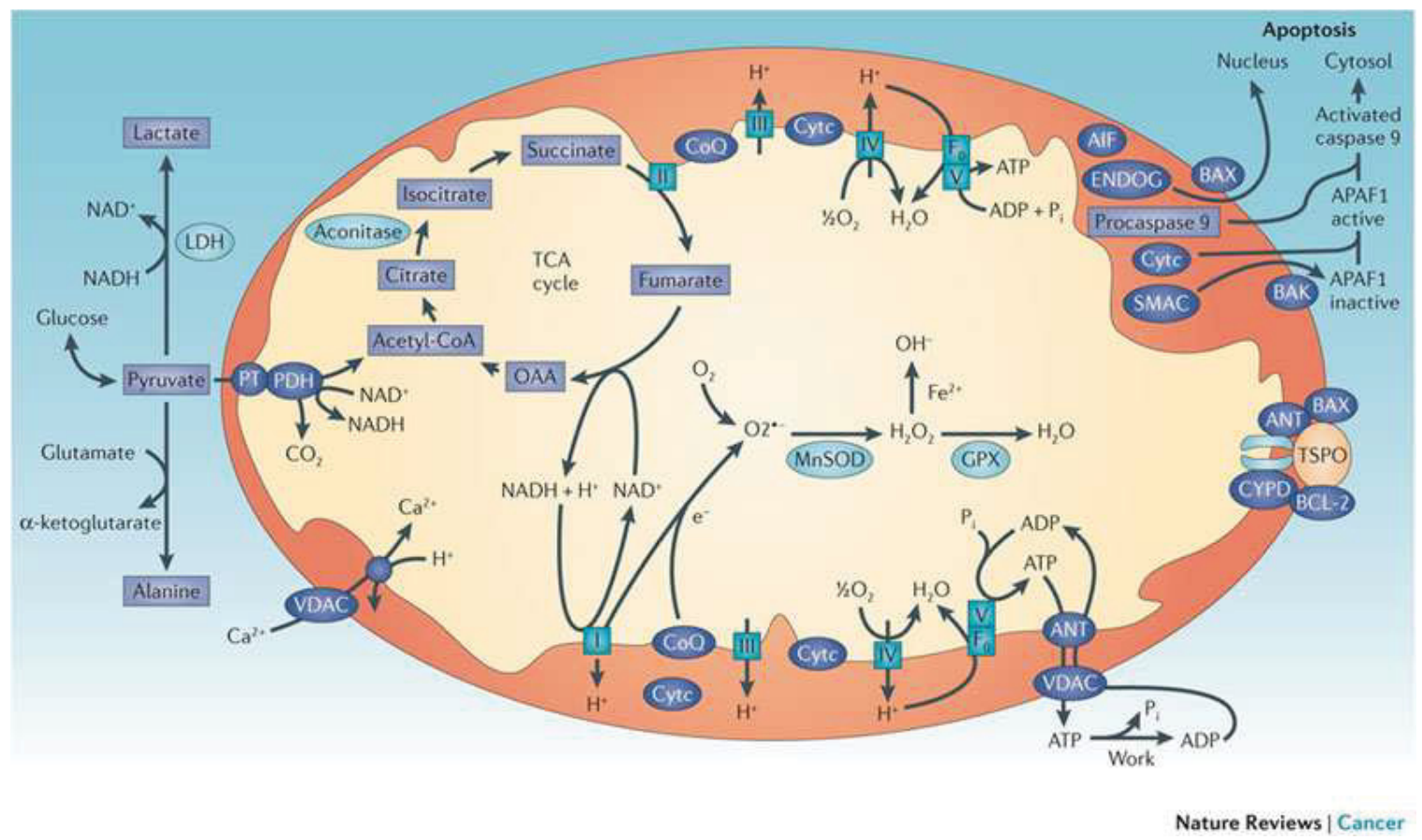


the sarcolemma or segregating at the ends of the nucleus. This strategic mitochondrial distribution allows for efficient ATP supply to high energy demanding, contracting cardiomyocytes (Milner et al. 2000).

As cardiac function is highly dependent upon ATP produced by mitochondrial oxidative phosphorylation, cardiac mitochondrial abnormalities leading to decreased ATP generation can cause cardiomyopathy, cardiac dysfunction, heart failure and even death (Antozzi and Zeviani 1997).

\section{Mitochondrial Cardiomyopathies}

The mitochondria are composed of more than 1000 polypeptides, mostly encoded by nDNA. However, thirteen polypeptides are encoded by the mtDNA, all of which are essential components of OXPHOS ETC complexes. They are seven of the 43 subunits of complex I (ND1, 2, 3, 4, 4L, 5, 6), one of the 11 subunits of complex III (cytb), three of the 13 subunits of complex IV (COXI, II, III), and two of the 16 subunits of complex V (ATP 6 and 8). The mtDNA also codes for the 12S and 16S rRNAs and 22 tRNAs, which are necessary for the synthesis of mitochondrial proteins (Wallace 2002; Wallace and Fan 2009). Mutation in mtDNA or nDNA, leading to mitochondrial protein deficiencies, can cause diseases with cardiomyopathy.

Cardiomyopathies are heart diseases that cause heart failure, arrhythmia and sudden death. Sequence change in both mitochondrial genome and nuclear genome can cause cardiomyopathies. Moreover, mutations of nuclear genes can not only cause the change of mtDNA structure and content, but also can directly affect the integrity and maintenance of mitochondrial ETC complexes, causing mitochondrial dysfunction and inducing disease (Schapira 2012). Of the 1000 genes encoding mitochondrial proteins, mutations in 228 protein-encoding nuclear DNA genes and $13 \mathrm{mtDNA}$ genes have been linked to human cardiomyopathies (Koopman et al. 2012).

Mutations in nDNA can also lead to mitochondrial-associated cardiomyopathies since most mitochondrial proteins are encoded by the nuclear genome. For example, mutations of the nuclear gene encoding NDUFV2 and NDUFS2 subunits for complex I have been strongly linked with early onset hypertrophic cardiomyopathy (Benit et al. 2003). Deletion of Ant1 in the heart also leads to the development of cardiomyopathy, since this abolishes exchange of ADP and ATP across mitochondrial membranes and dramatically increases ROS production. In addition, deletion of the Sod2 gene encoding ROS detoxification enzyme MnSOD leading to cardiomyopathy, indicates the importance of ROS in the pathogenesis of cardiomyopathy as ROS can cause mtDNA damage and inactivate iron-sulfur center on the ETC (Esposito et al. 1999). Recently, dilated cardiomyopathy induced by conditional deletion of optic atrophy-1 (Opa-1) and mitofusin (Mfn) 1/2 suggests that proteins regulating mitochondrial dynamics play an essential role in cardiac function (Chen et al. 2011; Chen et al. 2012; Dorn 2013). Furthermore, mutations in genes encoding contractile and structure proteins, such as $\beta$ myosin heavy chain, cardiac troponin $\mathrm{T}$ or $\mathrm{I}$, and myosin binding protein $\mathrm{C}$, have been 
reported to cause hypertrophy cardiomyopathy (Goldstein et al. 1999). Deficiency in the lipid content of mitochondria has also been implicated to cardiomyopathy. Barth syndrome, an X-linked cardiomyopathy, is caused by a defect in the cardiolipin transacylase enzyme, tafazzin. Optimal cardiolipin content is essential for proper function and organization of various mitochondrial enzymes. Mutations in tafazzin gene have been reported to result in reduced mitochondrial cardiolipin content. Patients show mitochondrial ultrastructural abnormalities, reduced OXPHOS, left ventricular dysfunction and dilation (Hauff and Hatch 2006; Schlame and Ren 2006; Claypool et al. 2008).

\section{Mitochondria and Heart Failure}

The involvement of mitochondria in the progression of heart failure was indicated as early as 1962 in a guinea pig model, in which reduced mitochondrial OXPHOS was observed (Schwartz and Lee 1962). It is well established that mitochondria-mediated cell death plays important roles in the pathogenesis of heart failure (covered previously). However, it is becoming more evident that mitochondria also contribute to the development of heart failure via mitochondrial ROS and calcium.

Since the heart is the most mitochondria enriched organ, mitochondrial-generated ROS is the major player of oxidative stress to the heart (Sommer and Waugh 1978). The toxic ROS have deleterious effects on mitochondrial and nuclear DNA, proteins, cell structure, and act as signaling molecules for the progression of heart failure. The mtDNA are more susceptible to ROS damage than nDNA since they have poor DNA repair mechanism and no protective histones. Moreover, $\mathrm{O}_{2}{ }^{--}$generated by OXOPHOS is not well membrane permeable and mostly is trapped within the mitochondria to cause the damage (Murphy 2009; Tsutsui et al. 2009). It is reported that the mutation rate of mtDNA is ten times more than the nDNA (Chen et al. 2006). There is evidence that ROS production is induced in patients with heart failure as well in animal models (Mallat et al. 1998; Ide et al. 2000). Therefore, strategies that upregulate the antioxidant defense pathways are candidates for the prevention or treatment of heart failure. Overexpression of the antioxidative enzyme Gpx can prevent the development of heart failure after myocardial infarction in mice (Shiomi et al. 2004). MitoQ ${ }_{10}$, an ubiquinol antioxidant, has been shown to be beneficial in protecting cardiac dysfunction and heart failure following ischemia/reperfusion injury (Adlam et al. 2005), hypertension and hypertrophy (Graham et al. 2009), sepsis-induced cardiac dysfunction (Supinski et al. 2009). In addition, dietary supplementation with vitamin $\mathrm{E}$ to rats can also protect against heart failure by increasing the activity of catalase and Gpx (Hill et al. 2005).

Mitochondrial calcium has been implied to mediate the opening of the mPTP to induce cell death to contribute to the pathogenesis of heart diseases, especially during ischemia/reperfusion injury. However, mitochondrial calcium also regulates energy metabolism to produce ATP. In cardiomyocytes, close apposition with the ER allows mitochondria to rapidly take up calcium released from ER. It is reported that an increase in mitochondrial calcium concentration results in activation of key mitochondrial 
metabolic enzymes, including pyruvate, $\alpha$-ketoglutarate and isocitrate dehydrogenases to increase NADH production and in turn ATP production (McCormack et al. 1990; Jouaville et al. 1999; Balaban 2002; Rosca and Hoppel 2010). In animal models with heart failure, there is a parallel reduction of ETC complexes activity and mitochondrial calcium uptake (Lin et al. 2007). Mitochondrial calcium also plays a role in regulating ROS. In animal models with heart failure, increasing mitochondrial calcium can reduce the induction of ROS. Conversely, the ROS production is enhanced in the presence of Ru360, which blocks mitochondrial calcium uptake. Cardiomyocytes isolated from the failing heart also show elevated mitochondrial ROS production and can be prevented by inhibiting mitochondrial sodium calcium exchanger (Kohlhaas et al. 2010). Thus, it is clear that mitochondrial ROS and calcium contribute to the development of heart failure. 


\section{CHAPTER 2. MATERIALS AND EXPERIMENTAL PROCEDURES*}

\section{Mice}

Mcl-1 conditional and Bax conditional Bak-/- mice have been described previously (Opferman et al. 2003; Takeuchi et al. 2005). Ckmm-Cre mice (obtained from The Jackson Laboratory, strain name B6.FVB(129S4)- $\mathrm{Tg}(\mathrm{Ckmm}$-cre) $5 \mathrm{Khn} / \mathrm{J})$ were described previously ( $\mathrm{Li}$ et al. 2000). $M y h$-Cre ${ }^{\mathrm{ER}}$ mice (obtained from The Jackson Laboratory, strain name B6.FVB(129)-Tg(Myh6-cre/ Esr1*)1Jmk/J) were described previously (Sohal et al. 2001). To induce deletion, mice received five doses of tamoxifen (1 mg per dose; Sigma) emulsified in sunflower oil vehicle (Sigma) by gavage. All mice were on a mixed 129/C57BL6 background and littermate controls were used. The mice were bred in accordance with the institutional animal care and use committee at St. Jude Children's Research Hospital. All mice used in the present studies were maintained in specific pathogen-free conditions and littermate controls were used as negative controls.

\section{Echocardiograph}

Mice were shaved and anesthetized with $0.5 \%-1.5 \%$ isoflurane in a $95 \% \mathrm{O}_{2}, 5 \%$ $\mathrm{CO}_{2}$ chamber prior to echocardiograph (ECHO). ECHO was performed using VEVO2100 high-frequency ultrasound instrumentation (Fujifilm VisualSonics, Inc.) equipped with a MS- 550D transducer operating at $40 \mathrm{MHz}$. All scans were taken with the heart rate between 300 and 400 beats per minute (bpm). M-Mode data were obtained in the parasternal short axis (PSAX) orientation to determine contractility of the heart. Data were post-processed using dedicated VisualSonics software (2100-1.4.0), and direct measurements were determined for IVS, LVPW, and LV dimensions at diastole and systole. From these data, EF and FS were determined to assess contractility.

\section{Histology}

Heart and skeletal muscle tissue was fixed in $10 \%$ neutral buffered formalin, embedded in paraffin, and cut into 4-um thick sections using HM500M cryostat (Microm). The paraffin sections were stained with hematoxlyn and eosin (H\&E) and Masson's trichrome stain. Paraffin sections were deparaffinized, and immunohistochemistry for detection of Capase-3 and Caspase-7 was performed using the following antibodies: anti- cleaved Caspase-3 and Caspase-7 (BioCare Medical).

* Reprinted with permission from Cold Spring Harbor Laboratory Press: Wang X, Bathina M, Lynch J, Koss B, Calabrese C, Frase S, Schuetz JD, Rehg JE, Opferman JT. 2013. Deletion of MCL-1 causes lethal cardiac failure and mitochondrial dysfunction. Genes \& development 27: 1351-1364. 
Anti-rabbit on rodent polymer (BioCare Medical) with deaminobenzidine detection system was used for visualization of the antigen. The number of total and positive nuclei was manually counted. Ten fields through the heart were analyzed for each animal under $20 \times$ magnification .

\section{Western Blotting and Antibodies}

Immunoblot analysis was performed as previously described (Stewart et al. 2010). Briefly, samples were lysed in radioimmunoprecifitation assay (RIPA) buffer $(50 \mathrm{mM}$ Tris- $\mathrm{HCl}$ at $\mathrm{pH} 7.4,150 \mathrm{mM} \mathrm{NaCl}, 1 \%$ TritonX-100, $1 \mathrm{mM}$ EDTA, $1 \%$ sodium deoxycholate, $0.1 \%$ SDS) containing protease inhibitors (Roche) on ice. Lysates were cleared by centrifugation, and protein concentrations were determined by BCA assay (Pierce). The following antibodies were used: anti-MCL-1 (Rockland Immunochemical), anti-BAX (Millipore), anti-BAK (Millipore), anti- Actin (Millipore), antiMnSOD (BD Biosciences), anti-Prohibitin (Thermo Fisher Scientific), anti-BIM (Millipore), anti-BNIP3 (Cell Signaling), and anti-BID (Cell Signaling).

\section{Isolation and Purification of Mouse Heart Mitochondria}

Mice were sacrificed with $\mathrm{CO}_{2}$, and beating hearts were re- moved. Hearts were rinsed by ice-cold mito isolation buffer (MIB; $200 \mathrm{mM}$ mannitol, $68 \mathrm{mM}$ sucrose, $10 \mathrm{mM}$ HEPES-KOH at pH 7.4, $10 \mathrm{mM} \mathrm{KCl}, 1 \mathrm{mM}$ EDTA, $1 \mathrm{mM} \mathrm{EGTA,} 0.1 \% \mathrm{BSA}$ ) to remove the blood and then finely minced. Minced tissue was homogenized with a Polytron tissue grinder at 12,000 rpm for $2.5 \mathrm{sec}$ and then hand-homogenized in a Potter-Helvehjem 10$\mathrm{mL}$ homogenizer (Teflon glass) for three to five strokes. Mitochondria were isolated by differential centrifugation. Briefly, the homogenized tissue was centrifuged twice at $600 \mathrm{~g}$ for $5 \mathrm{~min}$. The resulting pellet was resuspended in MIB and centrifuged at 5500g for 10 min. The final pellet was rinsed and gently homogenized in MIB. Concentration of mitochondria was determined by Bradford assay.

\section{Oxygen Consumption}

Mitochondria respiration was measured by a Clark-type oxygen electrode (Hansatech Oxygraph) in $500 \mu 1$ Mito Assay Buffer (MAB) (70 mM Sucrose, $220 \mathrm{mM}$ Mannitol, $10 \mathrm{mM} \mathrm{Pi}, 5 \mathrm{mM} \mathrm{Mgcl} 2,2 \mathrm{mM}$ Hepes, $1 \mathrm{mM}$ EGTA, $0.2 \% \mathrm{BSA}$ ) at $37^{\circ} \mathrm{C}$. Complex I respiration was stimulated by the addition of Glutamate $(5 \mathrm{mM})$ and malate $(2.5 \mathrm{mM})$. State 3 respiration was stimulated by the addition of ADP $(150 \mu \mathrm{M})$. State 4 respiration is the rate after adding oligomycin $(2 \mu \mathrm{g} / \mathrm{ml})$. Maximum respiration is obtained by the addition of FCCP $(0.4 \mu \mathrm{M})$. RCR is the ratio between state 3 and state 4 . 


\section{Enzymatic Assays}

The activity of complex I was assayed as previously described (Frost et al. 2005). Complex II activity was assayed by measuring succinate-dependent reduction in DCIP, mediated by CoQ1. The reaction was followed by absorbance at $600 \mathrm{~nm}$ following succinate addition (Miyadera et al. 2003). Complex IV activity was assayed by measuring oxidation of cytochrome $\mathrm{c}$ at $550 \mathrm{~nm}$ and calculated using the extinction coefficient of $19.6 \mathrm{mM}^{-1} \mathrm{~cm}^{-1}$ as previously described (Miro et al. 1998). Pyruvate dehydrogenase (PDH) activity was assayed by measuring the pyruvate-dependent reduction of INT, mediated by PMS, as previously described (Hinman and Blass 1981).

\section{DNA Isolation and Quantification}

DNA was extracted from the heart tissue of mice. To quantify the amount of mtDNA present per nuclear genome, we used the following primers:

mtDNA forward primer, 5'-CCTATCACCCTTGCCATCAT-3'; mtDNA reverse primer, 5'-GAGGCTGTTGCTTGTGTGAC-3'.

To quantify nuclear DNA, we used a primer set that detects the Pecam geneon chromosome 6:

nDNA forward primer, 5'-ATGGAAAGCCTGCCATCATG-3';

nDNA reverse primer, 5'-TCCTTGTTGTTCAGCATCAC-3'.

The relative copy number was quantified by analyzing the difference in threshold amplification between mtDNA and nuclear DNA. Real-time PCR was performed with SYBR Green (Bio-Rad) detection using a Bio-Rad iCycler using the ddCt method. The assay was done in triplicate for each sample.

To determine the leakiness of the $M y h-\mathrm{Cre}^{\mathrm{ER}}$, PCR were performed with the following primers:

Flox Mcl-1: 5'-CTGAGAGTTGTACCGGACAA-3';

Flox Mcl-1: 5'-GCAGTACAGGTTCAAGCCGATG-3'.

Deleted Mcl-1: 5'-CTGAGAGTTGTACCGGACAA-3';

Deleted Mcl-1: 5'-ACGCTCTTTAAGTGTTTGGCC-3'.

Cre: 5'-CTGCATTACCGGTCGATGCAAC-3';

Cre: 5'-TGCTAACCAGCGTTTTCGTTCTGCC-3'.

\section{RNA Isolation and Quantification}

RNA was extracted from heart tissue of mice by TRIzol (Ambion) and reverse transcribed by using SuperScript III first-strand synthesis system (Invitrogen). Real-time 
PCR with SYBR green (Bio-Rad) detection was performed using a BioRad icycler and the following primers:

$m P g c 1 \alpha$ forward: 5'-CCCTGCCATTGTAAAGAC-3'; $m P g c 1 \alpha$ reverse: 5'-TGCTGCTGTTCCTGTTTT-3'. mTfam forward: 5'-CATTTATGTATCTGAAAGCTTCC-3'; mTfam reverse: 5'-CTCTTCCCAAGACTTCATTTC-3'. $m$ Cpt $1 b$ forward: 5'-TCCAGTTGGCTTATCGTGGTG-3'; $m C p t 1 b$ reverse: 5'-CTAACGAGGGGTCGATCTTGG-3'. mMcad forward: 5'-GAGCAGGTTTCAAGATCGCA-3'; $m M c a d$ reverse: 5'-ACTTCGTGGCTTCGTCTAGA-3'. $m C o x 5 b$ forward: 5'-GCTGCATCTGTGAAGAGGACAAC-3'; $m$ Cox $5 b$ reverse: 5'-CAGCTTGTAATGGGTTCCACAGT-3'. $m$ Cox4 $i$ forward: 5'-ACCAAGCGAATGCTGAACAT-3'; $m$ Cox4i reverse: 5'-GGCGGAGAAGCCCTGAA-3'. mAtp synthase F1 forward: 5'-TCTCCATGCCTCTAACACTCG-3'; mAtp synthase Fl reverse: 5'-CCAGGGTCAACAGACGTGTCAG-3'. $m C y t c$ forward: 5'-CCAAATCTCCACGGTCTGTTC-3'; $m C y t c$ reverse: 5'-ATCAGGGTATCCTCTCCCCAG-3'. $m$ Catalase forward: 5'-GCAGATACCTGTGAACTGTC-3'; mCatalase reverse: 5'-GTAGAATGTCCGCACCTGAG-3'. mSod 1 forward: 5'-CCAGTGCAGGACCTCATTTT-3'; mSodl reverse: 5'-TCCCAGCATTTCCAGTCTTT-3'. mSod2 forward: 5'-GCACATTAACGCGCAGATCA-3'; mSod2 reverse: 5'-AGCCTCCAGCAACTCTCCTT-3'. mActin forward: 5'-GAGACCTTCAACACCCC-3'; mActin reverse: 5'-GTGGTGGTGAAGCTGTAGCC-3'.

ddCt method was used to calculate the mRNA level. The assay was done in triplicate for each sample.

\section{Transmission Electron Microscope}

Hearts were perfused and fixed in $2.5 \%$ gluteraldehyde in $0.1 \mathrm{M}$ sodium cacodylate buffer and post-fixed in $2 \%$ osmium tetroxide in $0.1 \mathrm{M}$ sodium cacodylate buffer with $0.3 \%$ potassium ferrocyanide. Tissue was stained with $4 \%$ aqueous uranyl acetate, dehydrated, infiltrated and embedded in epoxy resin. Ultra-thin sections $(80 \mathrm{~nm})$ were cut and imaged using a JEOL 1200 electron microscope with an AMT XR 111 camera. 


\section{Statistical Considerations}

Mendelian ratio significance was calculated using the Chi Square test; KaplanMeier curve significance was calculated using the Log-Rank test; ECG significance was calculated using the two-tailed Student $t$ test. $p$ values are denoted in figure legends 


\section{CHAPTER 3. RESULTS*}

\section{Cardiomyopathy Induced upon Constitutive Mcl-1-deletion in Cardiac and Skeletal Muscle}

To define the role of MCL-1 in the heart, we have generated a constitutive muscle specific $\mathrm{Mcl}-1$-deletion mouse model by crossing $\mathrm{Mcl}-\mathrm{I}^{\text {flox } / \text { flox }}$ mice with transgenic mice expressing Cre-recombinase driven by muscle creatine kinase $(\mathrm{Ckmm})$ promoter that expresses in both skeletal and cardiac muscle starting at embryonic day 13 (E13) (Li et al. 2000). The resulting $\mathrm{Mcl}-\mathrm{I}^{\text {flox/flox}}, \mathrm{Ckmm}$-Cre mice were born at the expected Mendelian ratio, but all pups died within the first 10 days after birth (Table 3-1 and Figure 3-1A). In contrast, no lethality was observed in wild-type (wt) or $\mathrm{Mcl}^{\mathrm{flox} / \mathrm{wt}}, \mathrm{Ckmm}$-Cre mice (Table 3-1 and Figure 3-1A). MCL-1 protein levels were significantly decreased by postnatal day 1 (P1) in the heart and skeletal muscle, but not in other tissues indicating that the deletion of Mcl-1 in this model is efficient and muscle-specific (Figure 3-1B). Histological analysis of the hearts of $\mathrm{Mcl}-\mathrm{I}^{\text {flox/flox }}, \mathrm{Ckmm}$-Cre mice at the age of P7 exhibited thinning of the heart walls, cardiac dilation, thrombus deposition, and interstitial fibrosis (Figure 3-1C). All of these features were absent in the littermate control mice indicating that deletion of $\mathrm{Mcl}-1$ elicited the myocardial degeneration. In contrast to the phenotypes observed in the $\mathrm{Mcl}$-1-deleted hearts, histological interrogation of skeletal muscle only revealed the rare appearance of sporadic myofiber necrosis and inflammation (Figure 3-1D). Transmission electron microscopy (TEM) analysis of $\mathrm{Mcl}$ 1-deleted skeletal muscle did not detect any overt abnormalities (Figure 3-2). Therefore, the effects of constitutive $\mathrm{Mcl}-\mathrm{l}$-deletion were most apparent in the cardiomyocytes with only subtle defects observed in skeletal muscle.

\section{Mcl-1-deletion in Adult Cardiac Muscle Results in Rapid, Fatal Cardiomyopathy}

Since the Ckmm-Cre mice express the Cre-recombinase during embryonic development, it is possible that the dramatic effects on myocardium could be due to developmental problems rather than acute damage to mature muscle. To test this hypothesis, we turned to an inducible, cardiac-specific $M c l-1$-deficient mouse model, in which $\mathrm{Mcl}-1$ deletion can be induced during adulthood. We crossed $M c l-I^{\text {flox/flox }}$ mice with Myh6-Mer-Cre-Mer $\left(M y h-\mathrm{Cre}^{\mathrm{ER}}\right)$ transgenic mice that express the tamoxifeninducible $\mathrm{Cr}^{\mathrm{ER}}$-recombinase under the control of a mouse, cardiac-specific $\alpha$-myosin heavy chain promoter (Sohal et al. 2001). $M c l-f^{\text {flox/flox }}, M y h-\mathrm{Cre}^{\mathrm{ER}}$ mice were born at the

* Reprinted with permission from Cold Spring Harbor Laboratory Press: Wang X, Bathina M, Lynch J, Koss B, Calabrese C, Frase S, Schuetz JD, Rehg JE, Opferman JT. 2013. Deletion of MCL-1 causes lethal cardiac failure and mitochondrial dysfunction. Genes \& development 27: 1351-1364. 
Table 3-1. Analysis of the progeny of $\mathrm{Ckmm}$-Cre-mediated deletion of $\mathrm{Mcl}-1$

\begin{tabular}{|c|c|c|c|c|}
\hline \multirow{3}{*}{ Genotype } & \multicolumn{4}{|c|}{$\mathrm{Mcl}-1^{\text {flox/flox }}$, wt X Mcl-1 ${ }^{\text {flox/wt }}, \mathrm{Ckmm}$-Cre } \\
\hline & \multicolumn{2}{|c|}{$\mathrm{P} 21$} & \multicolumn{2}{|c|}{ P1 } \\
\hline & Expected & Observed & Expected & Observed \\
\hline Mcl- $1^{\text {flox/flox }}, C \mathrm{kmm}$-Cre & 6 & 0 & 19 & 22 \\
\hline$M c l-1^{\text {flox/flox }}, \mathrm{wt}$ & 6 & 9 & 19 & 17 \\
\hline$M c l-1^{\text {flox/wt }}, C \mathrm{kmm}-\mathrm{Cre}$ & 6 & 8 & 19 & 17 \\
\hline$M c l-1^{\mathrm{flox} / \mathrm{wt}}, \mathrm{wt}$ & 6 & 7 & 19 & 20 \\
\hline Total & 24 & 24 & 76 & 76 \\
\hline Chi square analysis & \multicolumn{2}{|c|}{$p<0.02$} & \multicolumn{2}{|c|}{$p>0.05$} \\
\hline
\end{tabular}

Ckmm-Cre deletion of $M c l-1$ produces normal Mendelian ratios of newborn pups on day 1 after birth (P1), but these mice do not survive to weaning at day 21 after birth (P21). Expected and observed frequency of $\mathrm{Mcl}-1$ status in offspring from crosses of mice with the indicated genotypes. Chi square analyses results are indicated. 
A

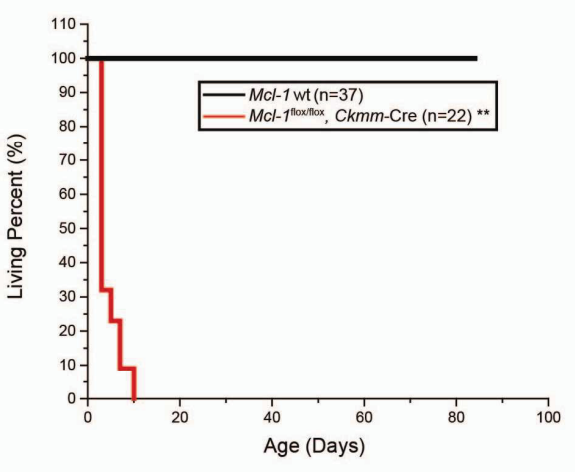

B

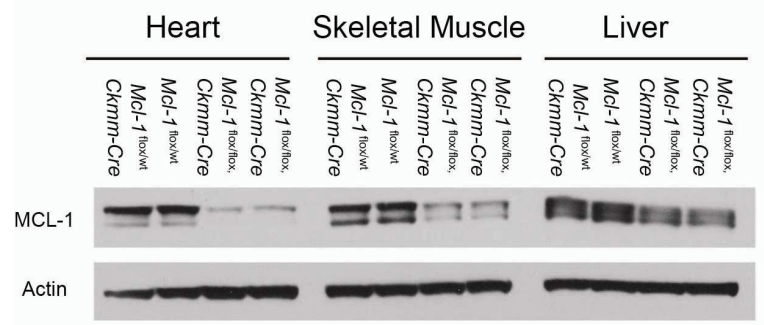

D

$\mathrm{MCl}-1^{\text {flox/wt }}$

$\mathrm{Mcl}-1^{\text {flox/flox, }}$

Ckmm-Cre

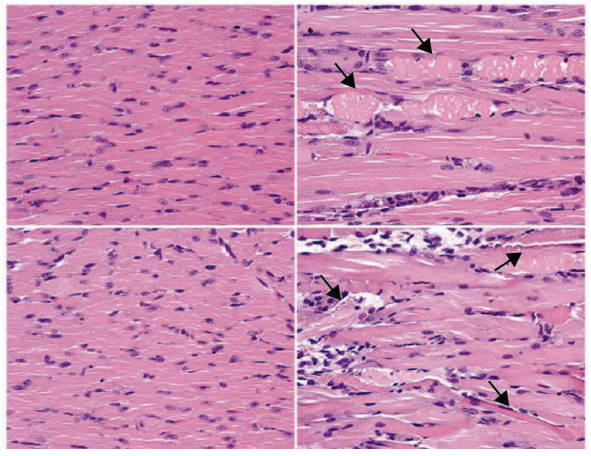

C

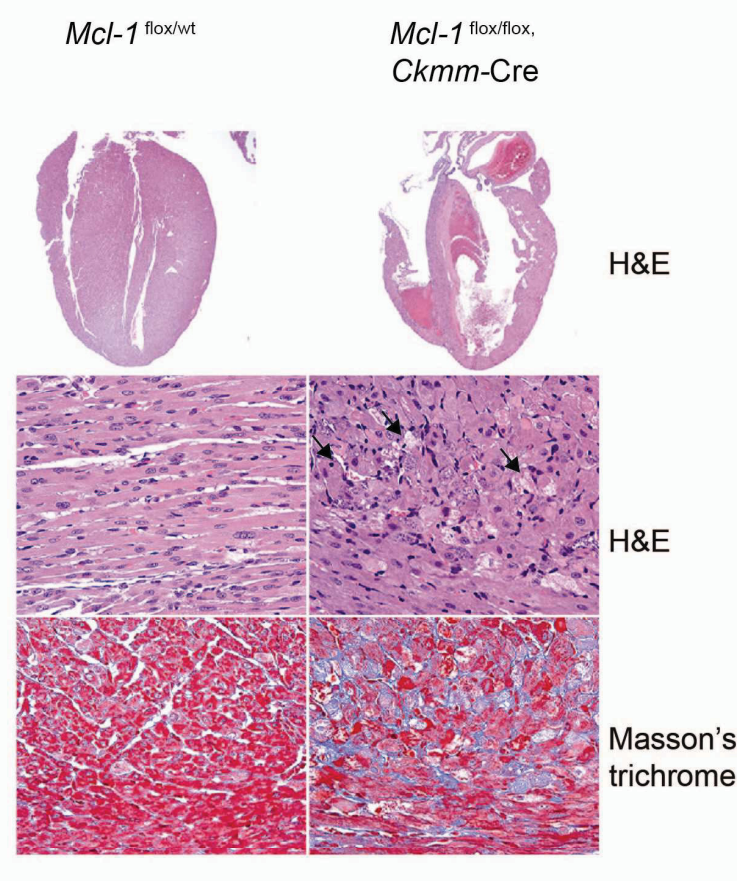

Figure 3-1. Cardiomyopathy is induced upon constitutive Mcl-1 deletion in cardiac and skeletal muscle

(A) Survival of muscle-specific Mcl-1-deleted mice. (**) $\mathrm{P}<0.01$ by log-rank test when compared with controls. (B) Constitutive $\mathrm{Mcl}-1$ deletion in heart and skeletal muscle. All tissues were harvested at P1 and immunoblotted with MCL-1 and Actin. (C) Hematoxylin and eosin (H\&E)-stained longitudinal sections of the heart indicate dilated cardiomyopathy and a loss of myofibrils. Connective tissue indicative of fibrosis stained blue with the Masson's trichrome stain. Hearts were harvested at P7. Magnifications: top, $2 \times$; middle and bottom, 40×. (D) H\&E-stained longitudinal sections of the tongue skeletal muscle indicate swollen fragmented fibers with hyaline necrosis. Magnification, $40 \times$. 
$\begin{array}{ll}M c l-1^{\text {flox/wt, }} & M c l-1^{\text {floxfllox }}, \\ \text { Ckmm-Cre } & C k m m-C r e\end{array}$

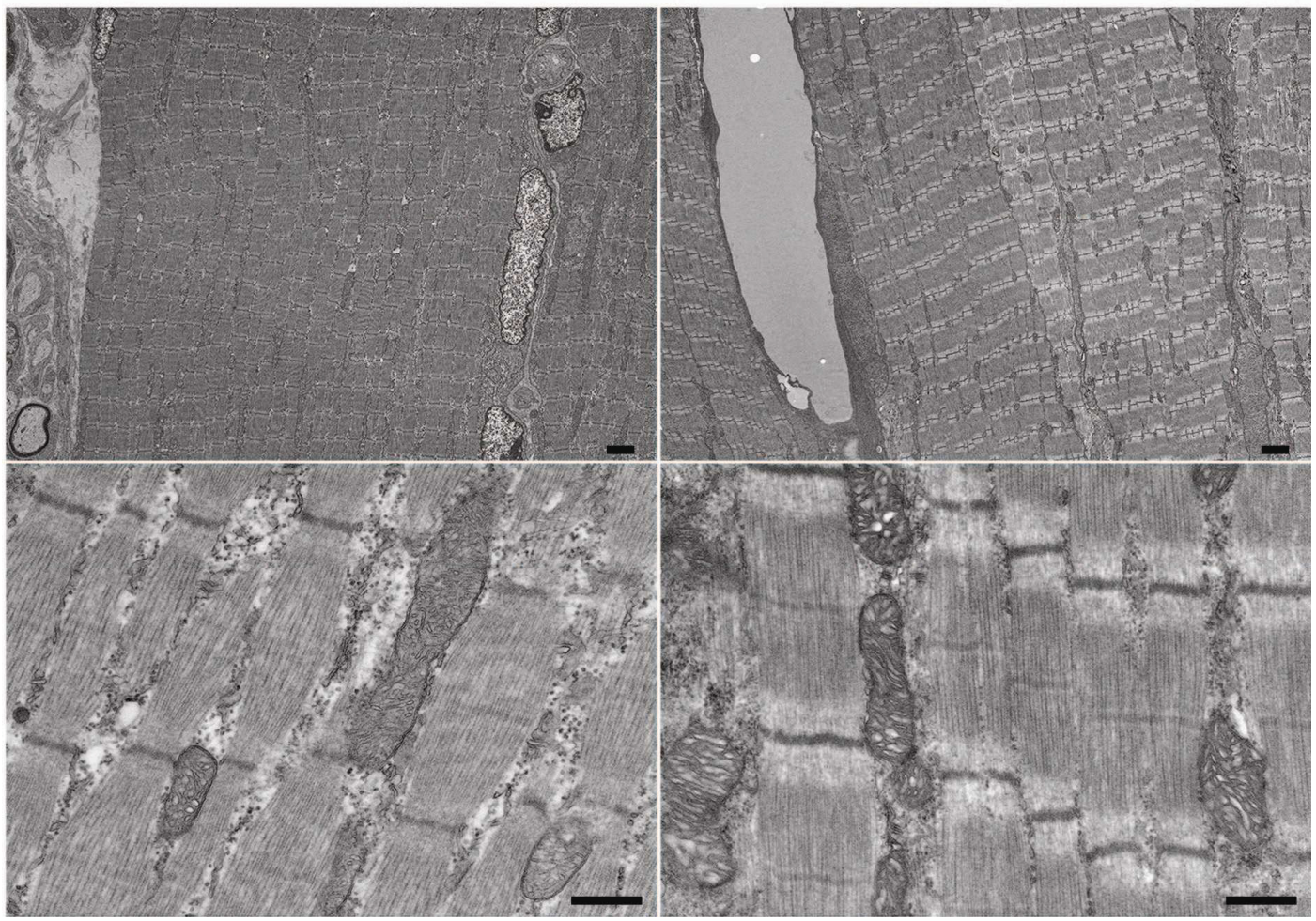

Figure 3-2. Mcl-1-deleted skeletal muscle revealed no overt evidence of myopathy Transmission electron micrographs indicated relatively normal skeletal muscle fibers and associated mitochondria ultrastructure comparable to littermate control. Scale bar represents: upper panel is $2 \mu \mathrm{m}$ and lower panel is $500 \mathrm{~nm}$. 
expected Mendelian ratio and exhibited no observable phenotype in the absence of tamoxifen treatment. However, two weeks after tamoxifen treatment of adult mice (aged 8-12 weeks), we observed a significant decrease of MCL-1 protein levels specifically in the hearts of $M c l-1^{\text {flox/flox }}, M y h-C r e^{\mathrm{ER}}$ mice, but not littermate control mice $\left(M c l-1^{\mathrm{wt} / \mathrm{wt}}\right.$, $M y h-\mathrm{Cre}^{\mathrm{ER}}$ ) (Figure 3-3A). Three weeks after tamoxifen initiation, echocardiographic analysis $(\mathrm{ECHO})$ detected dilated cardiomyopathy with increased left ventricle interior dimension (LVID), decreased left ventricle posterior wall (LVPW) and interventicular septum (IVS) wall thickness of cardiac specific $\mathrm{Mcl}$-1-deficient mice, but not littermate controls including $\mathrm{Mcl}-1^{\mathrm{wt} / \mathrm{wt}}, M y h-\mathrm{Cre}^{\mathrm{ER}}$ mice (Figure 3-3B). These data indicate that the lack of contractility is due to Mcl-1 deletion and not merely due to Cre activation. The left ventricle (LV) contractility as measured by ejection fraction (EF) and fraction shortening (FS) was significantly decreased and worsened with time after tamoxifentreatment (Figure 3-3C). Ultimately, the $M c l-1^{\text {flox/flox }}, M y h-C r e^{\mathrm{ER}}$ mice exhibited overt signs of heart failure 3 weeks after tamoxifen initiation and virtually all animals succumbed shortly thereafter to heart failure (Figure 3-3D). In a time-course experiment, we observed cardiac dysfunction in $M c l-1^{\text {flox/flox }}, M y h-C r e^{\mathrm{ER}}$ mice as soon as one week after tamoxifen administration (Figure 3-4A). Minor decreases in the cardiac function of $M c l-1^{\text {flox/flox }}, M y h-C r e^{\mathrm{ER}}$ mice prior to tamoxifen administration were consistent with a low level of recombination induced by $\mathrm{Cre}^{\mathrm{ER}}$ leakiness (Figure 3-4B). These data indicate that the cardiac dysfunction induced by $\mathrm{Mcl}$-1-deletion in adult mice is initiated quickly after deletion and rapidly leads to cardiac failure.

Consistent with the ECHO data, histological sections of $M c l-1^{\text {flox/flox }}, M y h-C r e^{\text {ER }}$ mice harvested 3 weeks after tamoxifen initiation displayed significantly dilated left ventricles and decreased wall thickness when compared with littermate control hearts (Figure 3-3E). Higher magnification histology revealed the loss of myofibrils in Mcl-1deleted hearts (Figure 3-3E). Furthermore, the regions with myofiber loss exhibited dramatic deposition of interstitial connective tissue as detected by Masson's trichrome staining (Figure 3-3F). These data indicate that similar to the constitutive deletion of $\mathrm{Mcl}-1$ in cardiac and skeletal muscle $(\mathrm{Ckmm}-\mathrm{Cre})$ that inducible deletion of $\mathrm{Mcl}-1$ in adult cardiac tissue $\left(M y h-\mathrm{Cre}^{\mathrm{ER}}\right)$ results in a rapid and fatal cardiomyopathy.

\section{Cardiac-specific Deletion of MCL-1 Induced Cell Death}

MCL-1 is an anti-apoptotic protein, and therefore it could be predicted that loss of MCL-1 expression promotes the induction of apoptosis, as has been observed in a variety of other tissues (Opferman et al. 2003; Opferman et al. 2005; Steimer et al. 2009). Within 1 wk after tamoxifen treatment, we observed that $\mathrm{Mcl}-1$ deletion results in the loss of cardiomyocytes and a corresponding increase in fibrosis. To test whether cardiac-specific deletion of MCL-1 induces apoptosis of the cardiomyocytes, we performed terminal deoxynucleotidyl transferase-mediated dUTP nick end labeling (TUNEL) and Caspase-3 immunohistochemical staining in constitutive ( $\mathrm{Ckmm}$-Cre) $\mathrm{Mcl}$-1-deleted hearts and inducible tamoxifen-treated $M y h-C \mathrm{Ce}^{\mathrm{ER}}$ hearts. Low but significant levels of apoptosis were observed in Mcl-1-deleted hearts in both mouse models (Figure 3-5). These data are consistent with previous reports that even low levels of cell death occurring in the heart 
Figure 3-3. Mcl-1 deletion in adult cardiac muscle results in rapid, fatal cardiomyopathy

(A) Tamoxifen-induced Mcl-1 deletion in the heart. All tissues were harvested 2 wk after tamoxifen initiation and immunoblotted with MCL-1 and Actin. (B) Cardiac function of a Mcl-1-deleted heart. Transthoracic ECHO studies were performed 3 wk after tamoxifen initiation. M-Mode images were recorded through IVS and LVPW at the papillary muscle level to get the LV dimensions and IVS and LVPW thickness. (C) Contractility of a Mcl-1-deleted heart as measured by ECHO for LV EF and FS. The data are presented as percent of baseline (before tamoxifen treatment). (**) $\mathrm{P}<0.01$. The number of mice analyzed is indicated as n. Note: Only twoMcl-1-deleted mice were analyzed at 6 wk due to the death of most animals by this time point. Error bars represent the standard error of the mean. (D) Survival of cardiac-specific Mcl-1-deleted mice. (**) P $<0.01$ by log-rank test. (E) H\&E-stained cross sections of the heart indicate dilated cardiomyopathy and a loss of myofibrils. Magnifications: left, 2×; right, 40×. (F) Fibrosis in the cross-sections of the heart stained blue with theMasson's trichrome stain. Magnifications: left, $2 \times$; right, $40 \times$. 


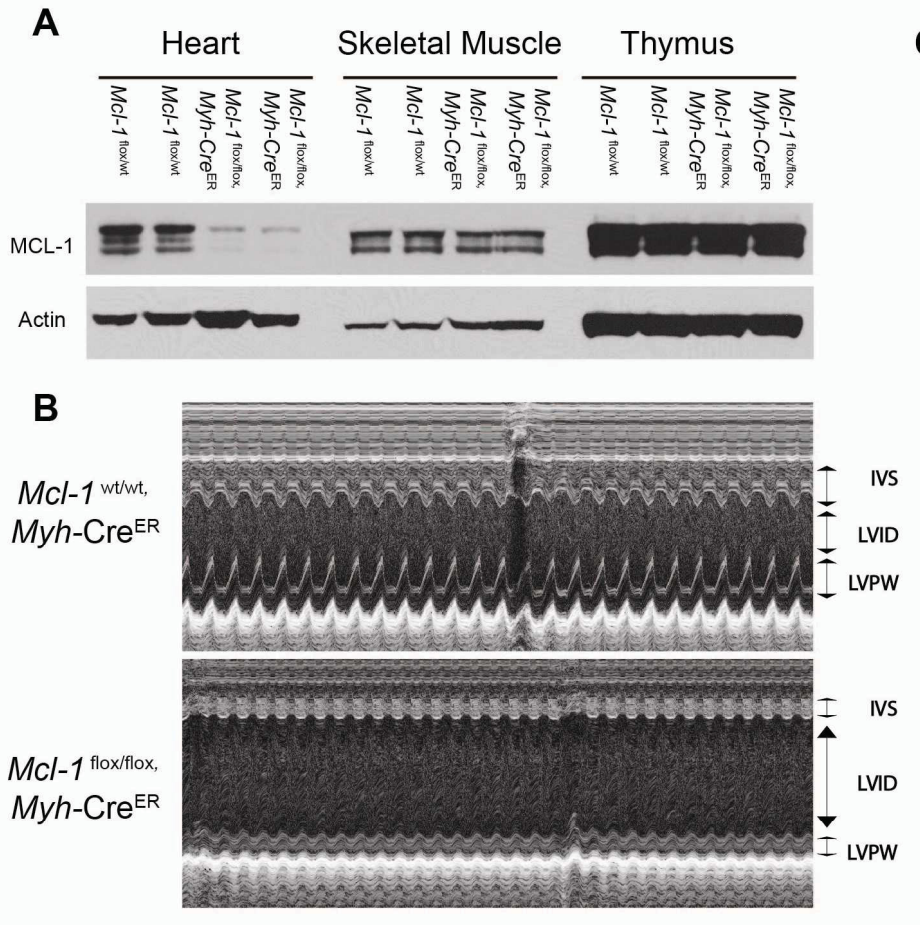

D

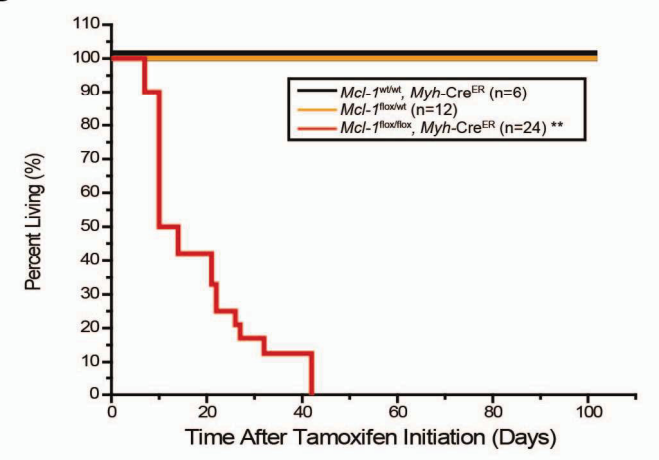

F

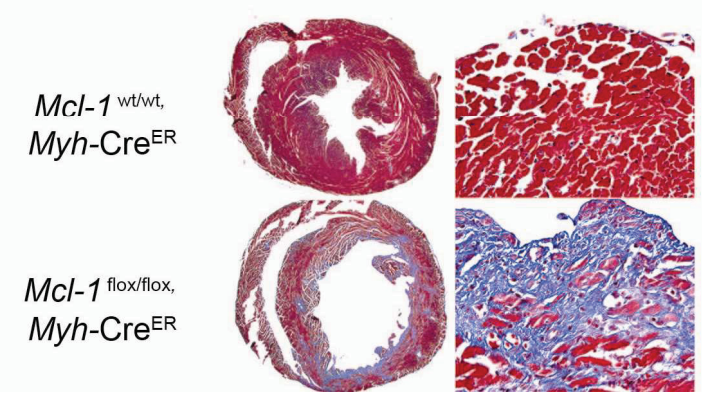

C
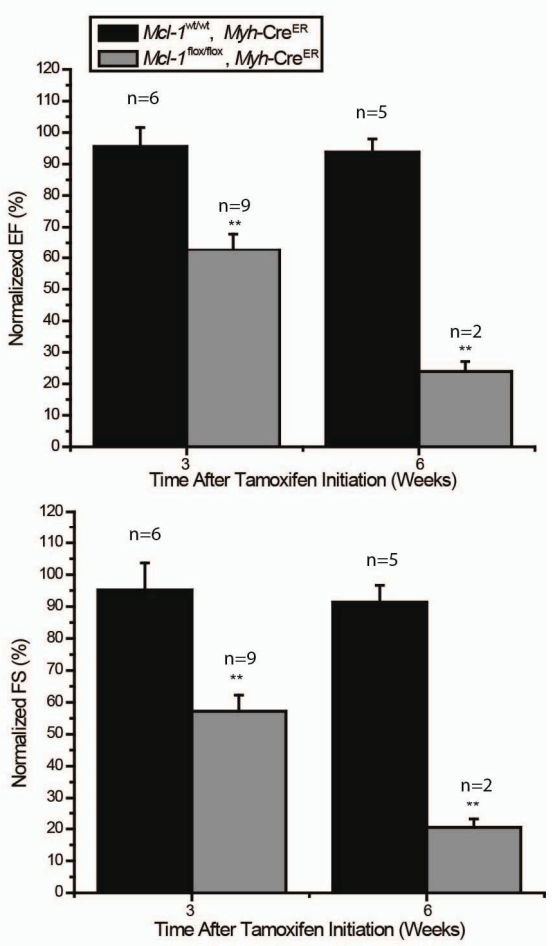

E

Mcl-1 1 wiwt, Myh-Cre ${ }^{\mathrm{ER}}$

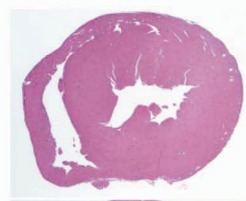

MCl-1 ${ }^{\text {floxfllox, }}$ Myh-Cre ${ }^{\mathrm{ER}}$

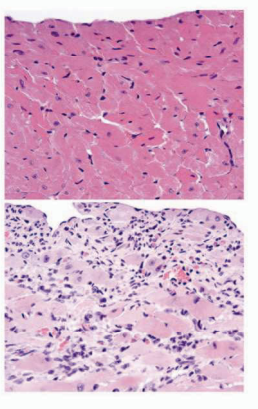




\section{A}
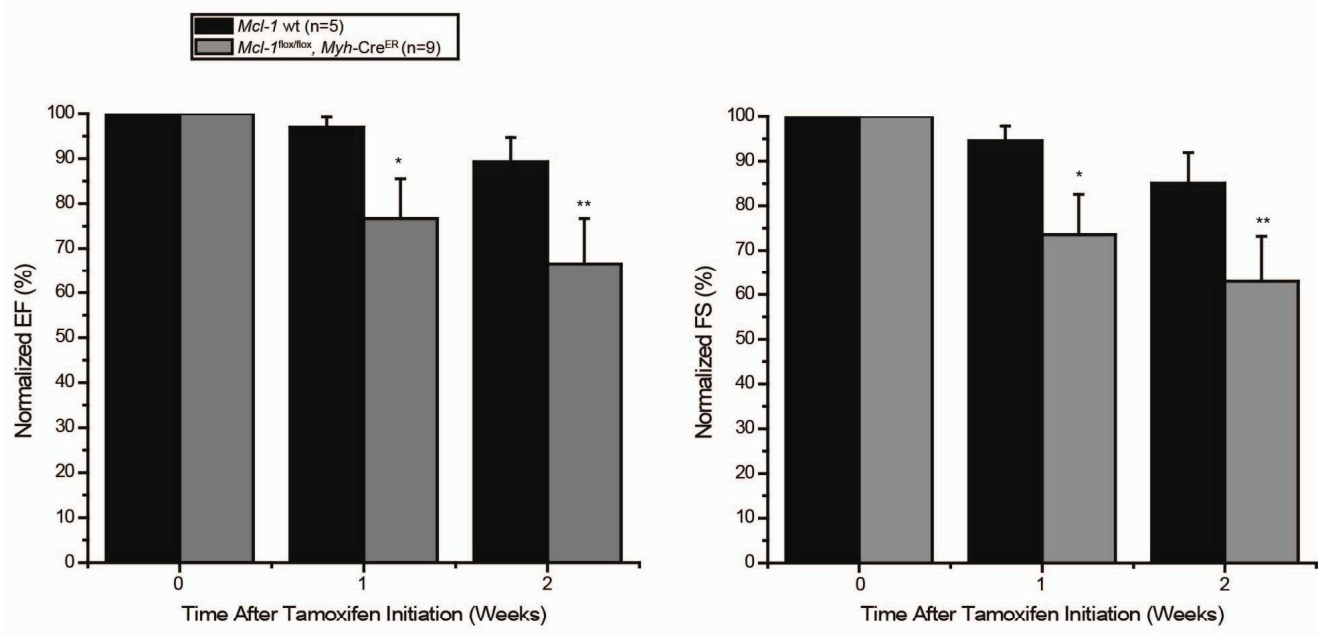

B

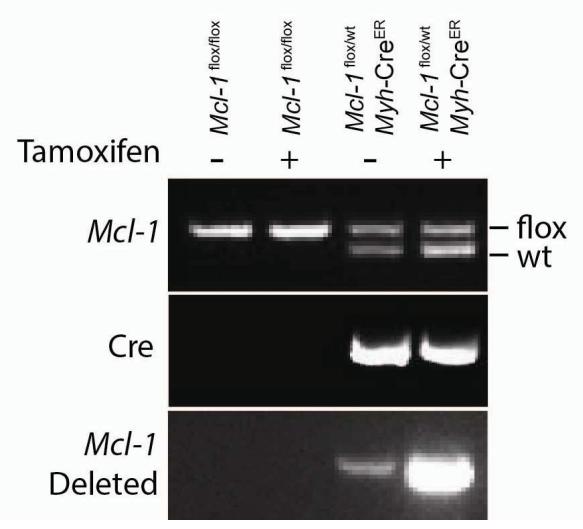

Figure 3-4. Kinetic analyses of cardiac dysfunction in $\mathrm{Mcl}-1$-deleted mice

(A) Earliest onset of cardiac dysfunction after Mcl-1-deletion. Transthoracic echocardiography was performed every seven days after tamoxifen initiation for indicated mouse strains. M-mode images were taken and left ventricle (LV) ejection fraction (EF) and fraction shortening (FS) were calculated. The data were presented as percent of baseline (before tamoxifen treatment). ${ }^{* *} p<0.01$. Error bars represent the standard error of mean. Control mice are a combination of $M c l-1^{\text {flox/wt }}$ and $M c l-I^{\text {flox/flox }}$, both without Cre. (B) Leakiness of the $M y h-\mathrm{Cre}^{\mathrm{ER}}$. DNA was extracted from heart tissue and PCR were performed. 
A

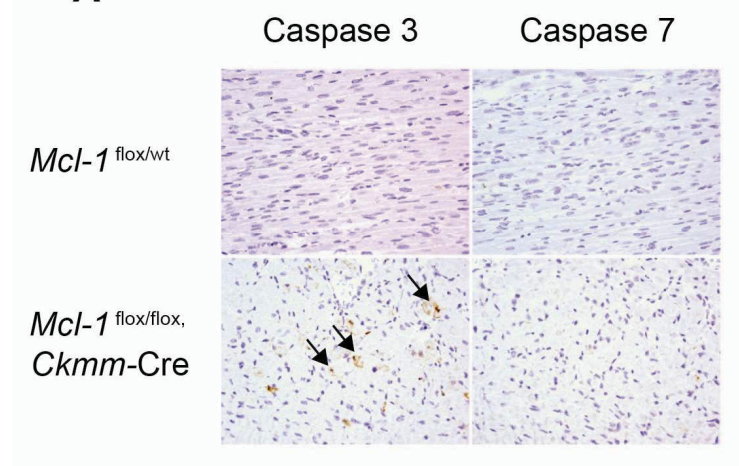

B

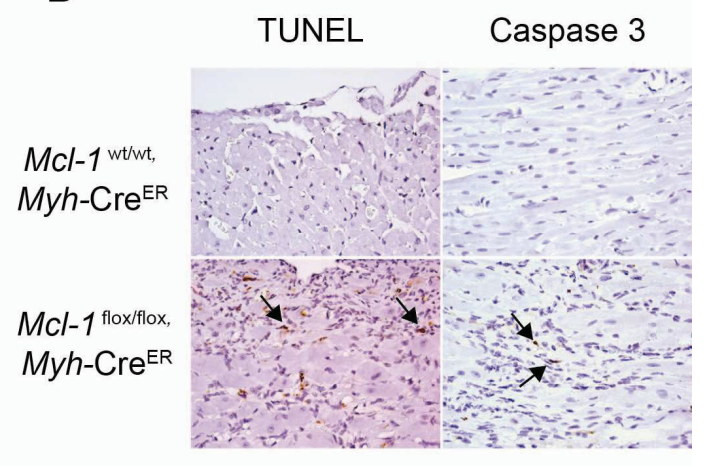

C
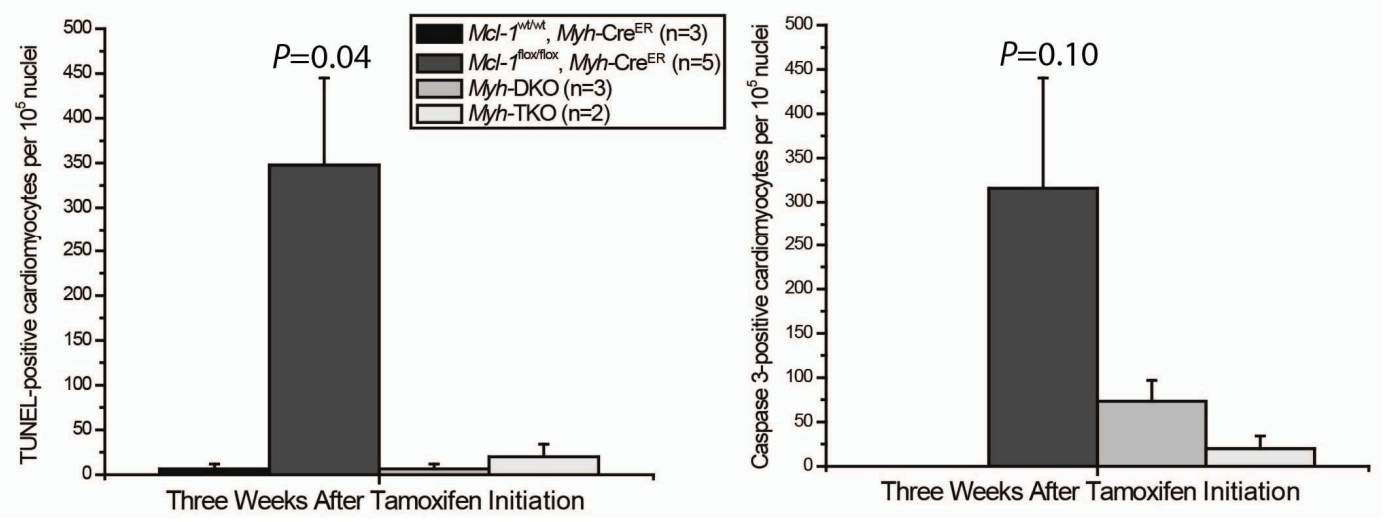

Three Weeks After Tamoxifen Initiation

\section{Figure 3-5. Cardiac-specific deletion of MCL-1 induced cell death}

(A) Caspase-3- and Caspase-7-stained sections of the heart indicate positive cells in constitutively $\mathrm{Mcl}$-1-deleted hearts (harvested at P7). Magnification, 40×. (B) TUNEL and Caspase-3 stained sections of the heart indicated positive cells in tamoxifen-induced Mcl-1-deleted hearts (three weeks after tamoxifen initiation). Magnification, 40×. (C) Number of TUNEL- and Caspase-3-positive cardiomyocytes upon Mcl-1 deletion. The number of mice analyzed is indicated as n. Error bars represent the standard error of the mean. The P-values are indicated comparing wild-type with Mcl-1-deleted mice by twotailed Student's t-test. 
are sufficient to result in cardiomyopathy, as adult cardiomyocytes are limited in their ability to regenerate after damage. (Kirshenbaum and Schneider 1995; Wencker 2003).

Anti-apoptotic MCL-1 functions in part by binding proapoptotic molecules, including BAX, BAK, BIM, PUMA, NOXA, and BID, on the mitochondria to prevent the induction of cell death (Certo et al. 2006; Willis et al. 2007). Immunoblot analyses of proapoptotic molecule expression indicated that upon $\mathrm{Mcl}-1$ deletion, the expression of most proapoptotic effectors at the mitochondria was unchanged, but both BAX and BIM levels were subtly elevated in $M c l-1$-deleted hearts (Figure 3-6). We were unable to detect significant expression of either PUMA or NOXA (data not shown), potentially as MCL-1 loss may be insufficient to induce the expression of these molecules in the heart, unlike overt stimuli like ischemia reperfusion (Toth et al. 2006). BNIP3, a molecule that has been implicated in cardiac cell death (Regula et al. 2002), was also found to be induced by $\mathrm{Mcl}-1$ deletion (Figure 3-6). Therefore, $\mathrm{Mcl}-1$ deletion is accompanied by an increase in expression of a number of proapoptotic proteins, including BAX, BIM, and BNIP3.

\section{Mitochondrial Ultrastructural Abnormalities and Respiratory Deficiency in Mcl-1- deleted Hearts}

Adequate cardiac function is dependent on an organized myocardium and mitochondria (Kuznetsov and Margreiter 2009); therefore, we analyzed the ultrastructure of Mcl-1-deleted hearts by TEM. In normal myocardium, mitochondria containing cristae with high electron density are elongated and tightly aligned between myofibrils (Milner et al. 2000). However, mitochondria in the Mcl-1-deleted myocardium from both mouse models appeared rounded, disorganized, and dispersed (Figure 3-7A, B). The cristae structure of mitochondria also appeared frequently fragmented, disrupted, and degraded, and many mitochondria possessed large vacuoles within an intact OMM (Figure 3-7A, B). These defects were similar to those observed in the mitochondria from $\mathrm{Mcl}$-1-deleted SV40-transformed MEFs (Perciavalle et al. 2012). Additionally, the loss of myofibrils detected by TEM was apparent from Mcl-1-deleted but not littermate control hearts (Figure 3-7A, B).

Mitochondrial cristae are enriched with proteins of the ETC that participate in mitochondrial oxidative phosphorylation and respiration (Da Cruz et al. 2003). Therefore, we sought to address whether the mitochondria isolated from Mcl-1-deleted cardiomyocytes exhibited defects in respiration by measuring the oxygen consumption rate (OCR). The mitochondria isolated from tamoxifen-treated $M c l-1^{\text {flox/flox }}, M y h$-Cre ${ }^{\mathrm{ER}}$ hearts revealed reduced state 3 respiration (ADP-stimulated) when compared with littermate control mitochondria. The respiratory control ratio (RCR) of the $M c l-1$-deleted mitochondria was significantly lower than that of littermate controls (Table 3-2). Furthermore, the maximal respiration of $\mathrm{Mcl}$-1-deleted mitochondria (FCCP-uncoupled) was also reduced when compared with littermate controls (Table 3-2). Last, Mcl-1 deletion caused individual ETC complex enzymatic activity for NADH ubiquinone oxidoreductase (complex I) and cytochrome c oxidase (complex IV) to be reduced when 


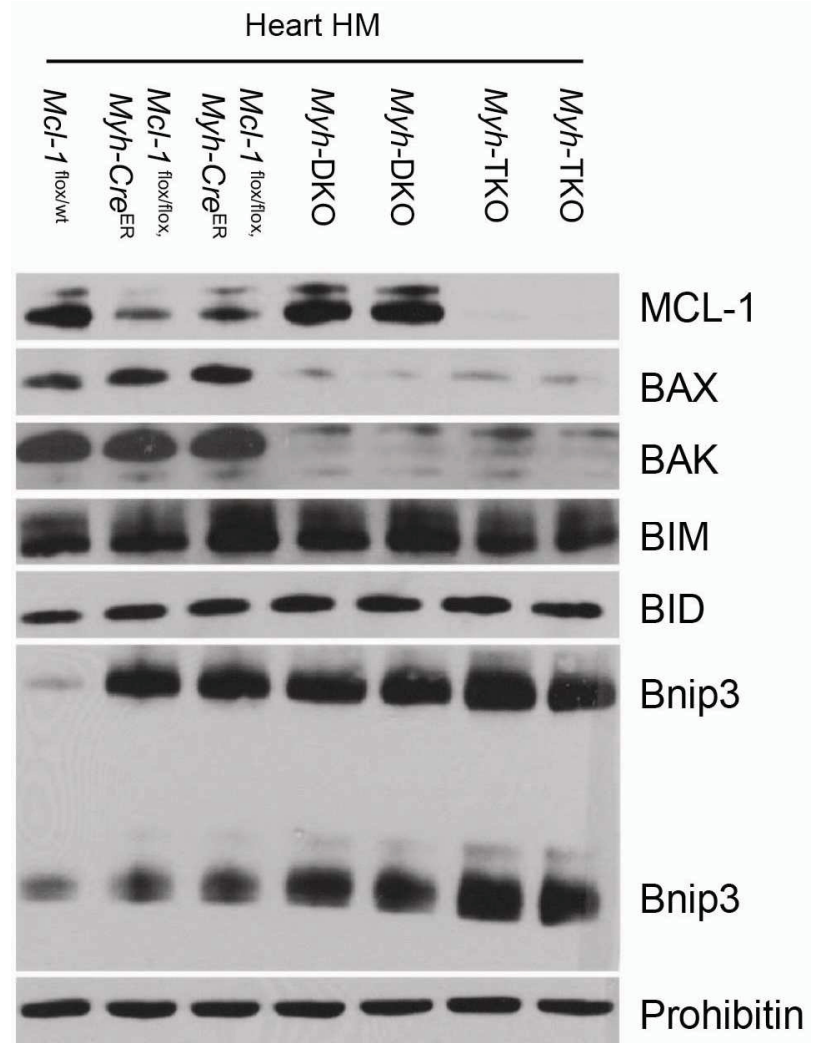

Figure 3-6. Expression of pro-apoptotic molecules in the cardiac mitochondria Cardiac mitochondria were isolated from tamoxifen-treated $\mathrm{Mcl}-1^{\mathrm{wt} / \mathrm{wt}}, \mathrm{Myh}-\mathrm{Cre}{ }^{\mathrm{ER}}, \mathrm{Mcl}$ $l^{\text {flox/flox }}, M y h$-Cre ${ }^{\mathrm{ER}}, M y h$-DKO and $M y h$-TKO mice and blotted with indicated antibodies. Prohibitin serves as loading control. 


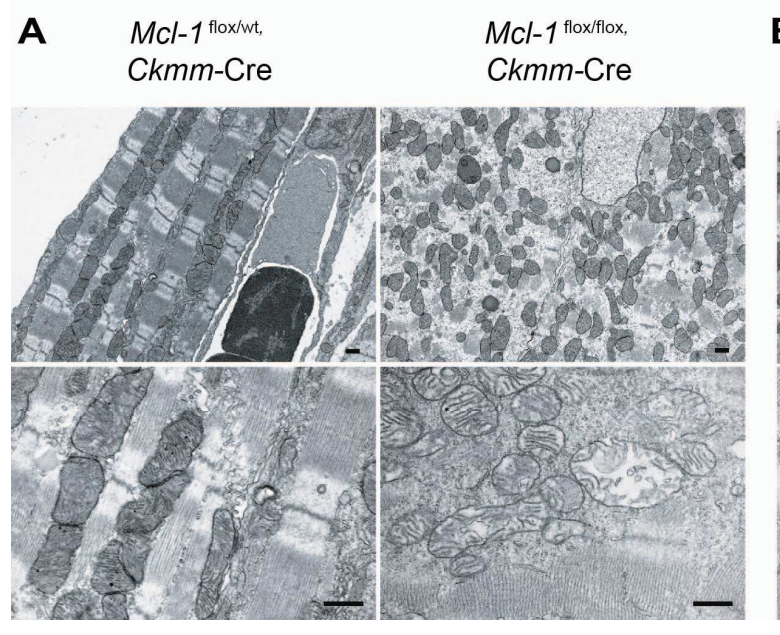
B $\quad M C l-1$ wtint, $\quad M C l-1$ floxflox,
Myh-Cre ${ }^{\mathrm{ER}} \quad$ Myh-Cre ${ }^{\mathrm{ER}}$

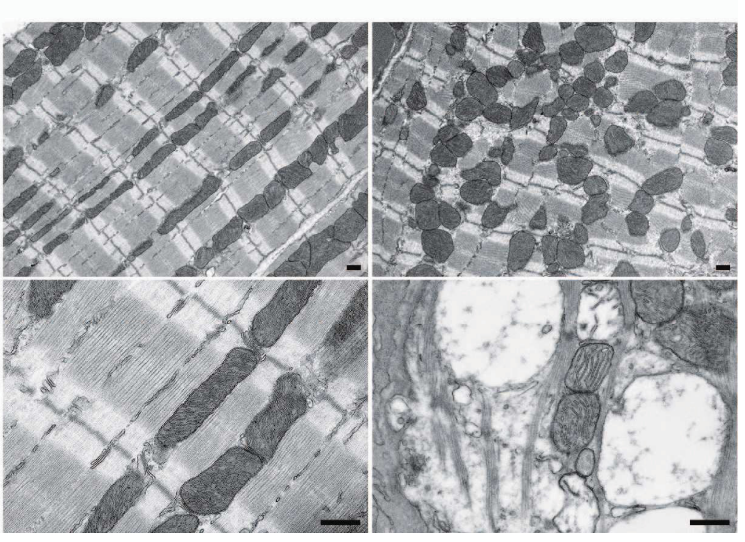

Figure 3-7. Mitochondrial ultrastructural abnormalities and respiratory deficiency in $\mathrm{Mcl}-1$-deleted hearts

Transmission electron micrographs indicate disrupted myocardium and disorganized mitochondria with abnormal cristae structure in (A) Constitutively Mcl-1-deleted hearts (harvested at P9). Bar, $500 \mathrm{~nm}$. (B) Tamoxifen-induced Mcl-1-deleted hearts (3 wk after tamoxifen initiation).Bar, $500 \mathrm{~nm}$. 
Table 3-2. Oxygen consumption of isolated cardiac mitochondria

\begin{tabular}{|c|c|c|c|c|}
\hline \multirow{2}{*}{ Genotype } & \multicolumn{4}{|c|}{ Glutamate/malate } \\
\hline & STATE 3 & STATE 4 & $\mathrm{RCR}$ & FCCP \\
\hline$M c l-I^{\text {flox/wt }}(\mathrm{n}=6)$ & $400.5 \pm 29.8$ & $94.5 \pm 7.9$ & $4.3 \pm 0.1$ & $408.3 \pm 27.2$ \\
\hline$M c l-1^{\text {flox/flox }}, M y h-\mathrm{Cre}^{\mathrm{ER}}(\mathrm{n}=3)$ & $239.9 \pm 9.7 *$ & $83.8 \pm 6.0$ & $2.9 \pm 0.1 * *$ & $236.2 \pm 7.6 * *$ \\
\hline$M y h-\mathrm{DKO}(\mathrm{n}=3)$ & $485.8 \pm 55.5$ & $121.3 \pm 14.3$ & $4.0 \pm 0.2$ & $522.6 \pm 62.5$ \\
\hline$M y h-$ TKO $(\mathrm{n}=3)$ & $287.2 \pm 4.5^{*}$ & $100.9 \pm 15.3$ & $3.0 \pm 0.4^{* *}$ & $262.7 \pm 21.1^{*}$ \\
\hline
\end{tabular}

Oxygen consumption of mitochondria isolated from $M c l-1^{\text {flox/wt }}$ and $M c l-1^{\text {flox/flox }}, M y h$ $\mathrm{Cre}^{\mathrm{ER}}$ hearts were 2 weeks after tamoxifen-treatment and from $M y h$-DKO and $M y h$-TKO hearts isolated 8 weeks after tamoxifen-treatment. Glutamate $(5 \mathrm{mM})$ and malate $(2.5$ $\mathrm{mM})$ are substrates for Complex I. State 3 respiration is stimulated by ADP $(150 \mu \mathrm{M})$. State 4 respiration is the rate after adding oligomycin $(2 \mu \mathrm{g} / \mathrm{ml})$. Maximum respiration is the rate after adding FCCP $(0.4 \mu \mathrm{M})$. RCR is the ratio of state 3 and state $4 .{ }^{*} p<0.05$, ${ }^{* *} p<0.01$ when compared to $M c l-1^{\text {flox/wt }}$ mitochondria. For each group, (n) indicates the number of individual animals used for mitochondrial isolation and each measurement was done in duplicate. 
assessed from isolated mitochondria but did not alter the function of succinate ubiquinone oxidoreductase (complex II) (Figure 3-8A). The decreased function of the ETC components was not due to differential purification, as similar levels of mitochondrial (Prohibitin and MnSOD) and endoplasmic reticulum (BiP) contaminants were found in the preparations (Figure 3-8B). These data suggest that MCL-1 is necessary for the maintenance of normal cardiac mitochondrial respiration.

Mitochondrial dysfunction can result in elevated production of ROS (Wallace 1999). ROS are well known to induce mtDNA mutations or deletions that have been implicated to cause cardiomyopathy (Fosslien 2003). Therefore, we investigated whether we could detect alterations in the mitochondrial genome by quantitative real-time PCR (qPCR). Consistent with previous observations made in Mcl-1-deleted MEFs and livers (Perciavalle et al. 2012), the induction of cardiac-specific Mcl-1 deletion resulted in decreased mtDNA levels when normalized to genomic DNA (Table 3-3). Given the drop in mtDNA, we analyzed whether reductions in the total mitochondria number in the deleted hearts could be detected. Importantly, we did not observe major differences in the total amount of mitochondrial proteins or citrate synthase enzymatic activity in Mcl-1deleted and littermate control hearts, indicating that the loss of MCL-1 did not simply lead to a dramatic loss of total mitochondrial mass (Figure 3-8B, C). Furthermore, there was no detectable induction of a PGC1- $\alpha$-dependent mitochondrial biogenesis program in the Mcl-1-deleted hearts; instead, PGC1- $\alpha$ target genes were actually repressed (Figure 3-9). Such a repression of PGC1- $\alpha$ target genes has also been observed in several models of cardiac hypertrophy and failure (Lehman and Kelly 2002; Garnier et al. 2003; Arany et al. 2006). Thus, these data indicate that $\mathrm{Mcl}-1$ deletion results in mitochondrial dysfunction, which we predict contributes to the cardiomyopathy and decreased mitochondrial respiration in our model.

\section{Genetic Ablation of $B a x$ and $B a k$ Improves Cardiac Function and Survival of Cardiac-specific $\mathrm{Mcl}$-1-deletion}

To further confirm that apoptosis contributed to the cardiac pathology after Mcl-1 deletion, we sought to genetically disable the intrinsic apoptotic pathway in the mouse heart by deleting both proapoptotic effectors BAX and BAK (Lindsten et al. 2000; Wei et al. 2001). To do so, $M c l-1^{\text {flox/flox }}$ mice were bred to $B a x^{\text {flox/flox }}, B a k$-deficient mice to produce mice in which Mcl-1, Bax, and Bak could simultaneously be genetically deleted using either $C k m m$-Cre or, $M y h-C r e^{\mathrm{ER}}$ (Takeuchi et al. 2005). In addition, deletion of Bax and Bak may also down-regulate cell death caused by primary necrosis (opening of mPTP in the absence of cytochrome $\mathrm{c}$ release) by increasing the threshold for the opening of the mPTP (Whelan et al. 2012). Therefore, loss of BAX and BAK could be anticipated to block both necrotic and apoptotic cell death.

$M c l-1^{\text {floxflox }}, B a x^{\text {flox flox }}, \mathrm{Bak}^{-/ 2}, \mathrm{Ckmm}$-Cre (hereafter referred to as $\mathrm{Ckmm}$ triple knockout [TKO]) mice were born at the expected Mendelian ratios and, unlike $\mathrm{Mcl}$ -

$I^{\text {flox/flox }}, C \mathrm{kmm}$-Cre, were able to survive to adulthood (Figure 3-10A). Analysis of the one mouse out of $10 \mathrm{Ckmm}$-TKO mice that revealed cardiac dysfunction at $5 \mathrm{wk}$ of age 
A

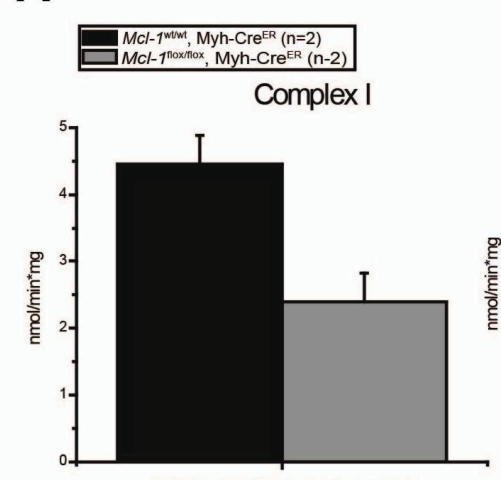

Two Weeks After Tamoxifen Initiation

B

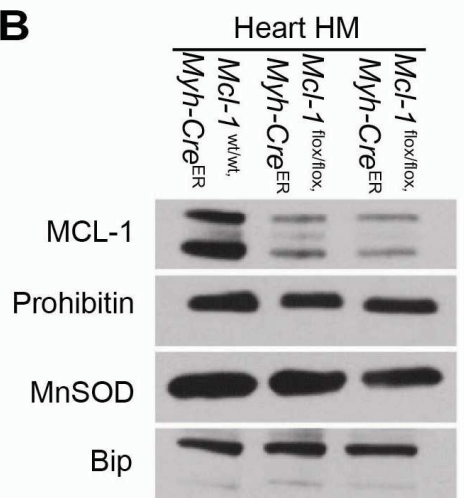

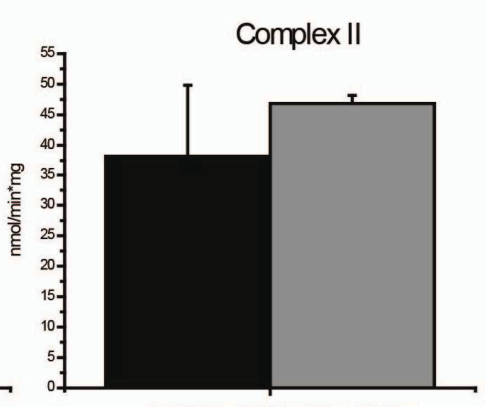

Two Weeks After Tamoxifen Initiation

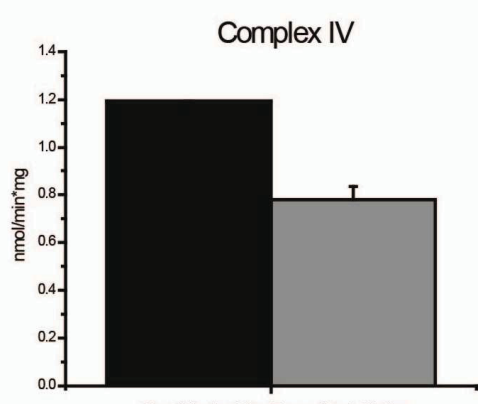

Two Weeks After Tamoxifen Initiation

C

Citrate Synthase

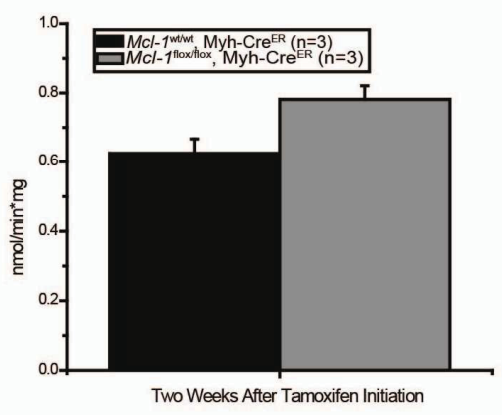

Figure 3-8. Enzymatic function of $\mathbf{M c l}-1$-deleted mitochondria

(A) Enzymatic activity assays for indicated electron transport chain components for mouse cardiac mitochondria isolated from $M c l-I^{\mathrm{wt} / \mathrm{wt}}, M y h-\mathrm{Cre}^{\mathrm{ER}}, M c l-I^{\text {flox/flox }}, M y h-$ $\mathrm{Cre}^{\mathrm{ER}}, M y h$-DKO and $M y h$-TKO mice after tamoxifen treatment. Error bars represent the standard error of mean. (B) Purity of the isolated cardiac mitochondria was indicated by mitochondria marker Prohibitin and MnSOD. BIP serves as ER marker. (C) Citrate synthase activity in whole heart homogenates from $M c l-I^{\mathrm{wt} / \mathrm{wt}}, M y h-\mathrm{Cre}^{\mathrm{ER}}$ and $\mathrm{Mcl}$ $I^{\text {flox/flox }}, M y h-\mathrm{Cre}^{\mathrm{ER}}$ mice after tamoxifen treatment. Error bars represent the standard error of mean. 
Table 3-3. Quantification of mitochondrial DNA from isolated cardiac muscle

\begin{tabular}{lc}
\hline \multicolumn{1}{c}{ Genetype } & Fold Change vs. $M c l-1^{\text {flox/wt }},(w t)$ no Cre \\
\hline$M c l-1^{\text {flox } / w t}$, wt $(\mathrm{n}=5)$ & 1 \\
$M c l-1^{\text {flox } / \text { llox }}, M y h-C r e^{\text {ER }}(\mathrm{n}=4)$ & $0.75 \pm 0.086$ \\
$M y h-D K O(\mathrm{n}=3)$ & $0.97 \pm 0.192$ \\
$M y h-\mathrm{TKO}(\mathrm{n}=2)$ & $0.82 \pm 0.01$ \\
\hline
\end{tabular}

Cardiac muscle fiber was isolated after tamoxifen-treatment and assessed for relative mitochondrial DNA and genomic DNA. The $M c l-1^{\text {flox/wt }}$ and $M c l-1^{\text {flox/flox }}, M y h-C r e^{\text {ER }}$ hearts were harvested 2 weeks after tamoxifen-treatment while the $M y h$-DKO and $M y h$ TKO hearts were harvested 8 weeks after tamoxifen-treatment. The ratio of mtDNA and nDNA by quantitative real-time PCR is presented with the error representing the standard error of the mean. For each group, (n) indicates the number of individual animals used for DNA isolation and each measurement was done in triplicate. 

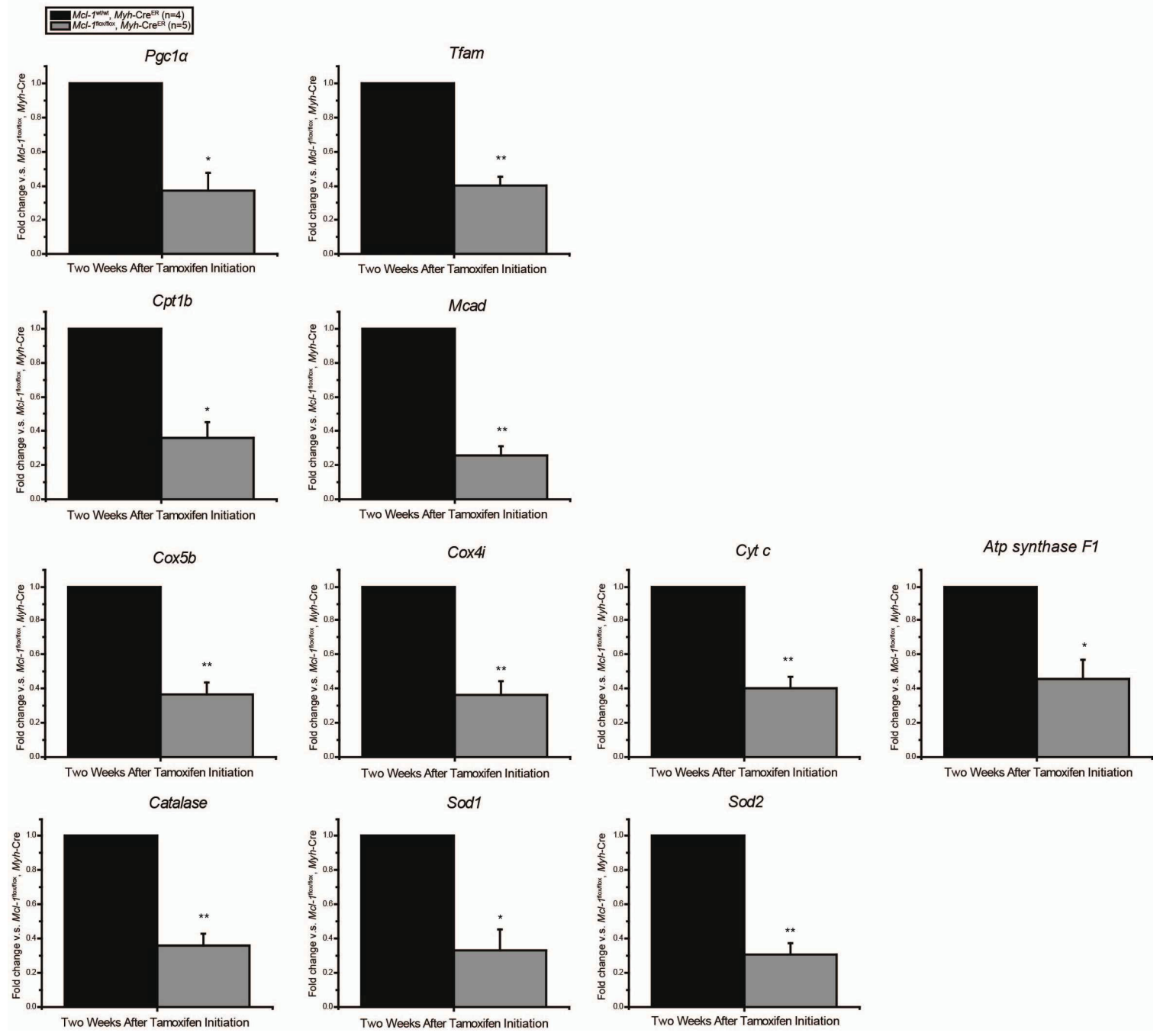

Figure 3-9. mRNA level of PGC1-alpha and its target genes

Tfam gene encodes a mitochondrial transcription factor that is a key activator for mitochondrial genome transcription. Cpt $1 b$ and $M a c d$ genes encode enzymes involved in the fatty acid $\beta$-oxidation. Cox $5 b, \operatorname{Cox} 4 i$, cyt $c$ and Atp synthase F1 genes encode proteins of the electron transport chain. Catalase, Sod1 and Sod2 genes encode antioxidative proteins. RNA was extracted from heart tissue of mice by TRIzol (Ambion) and reverse transcribed by using SuperScript III first-strand synthesis system (Invitrogen). Real-time PCR with SYBR green (Bio-Rad) detection was performed using a BioRad icycler. ddCt method was used to calculate the mRNA level. The assay was done in triplicate for each sample. ${ }^{*} p<0.05,{ }^{*} p<0.01$ as determined by two-tailed Student $t$ test. Error bars represent the standard error of mean. 

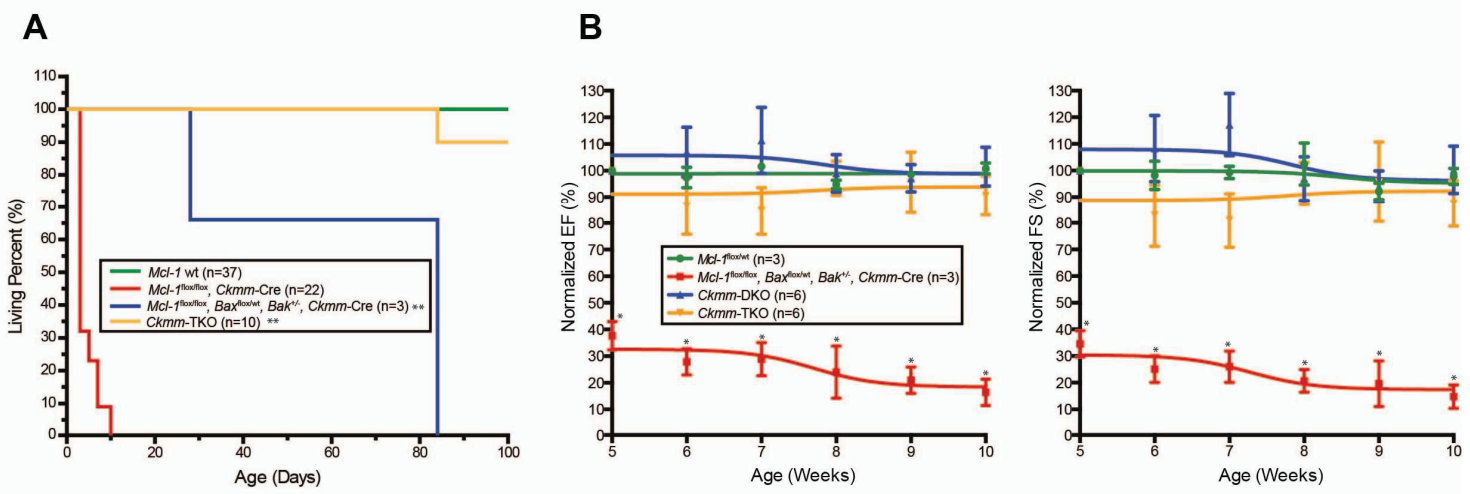

C

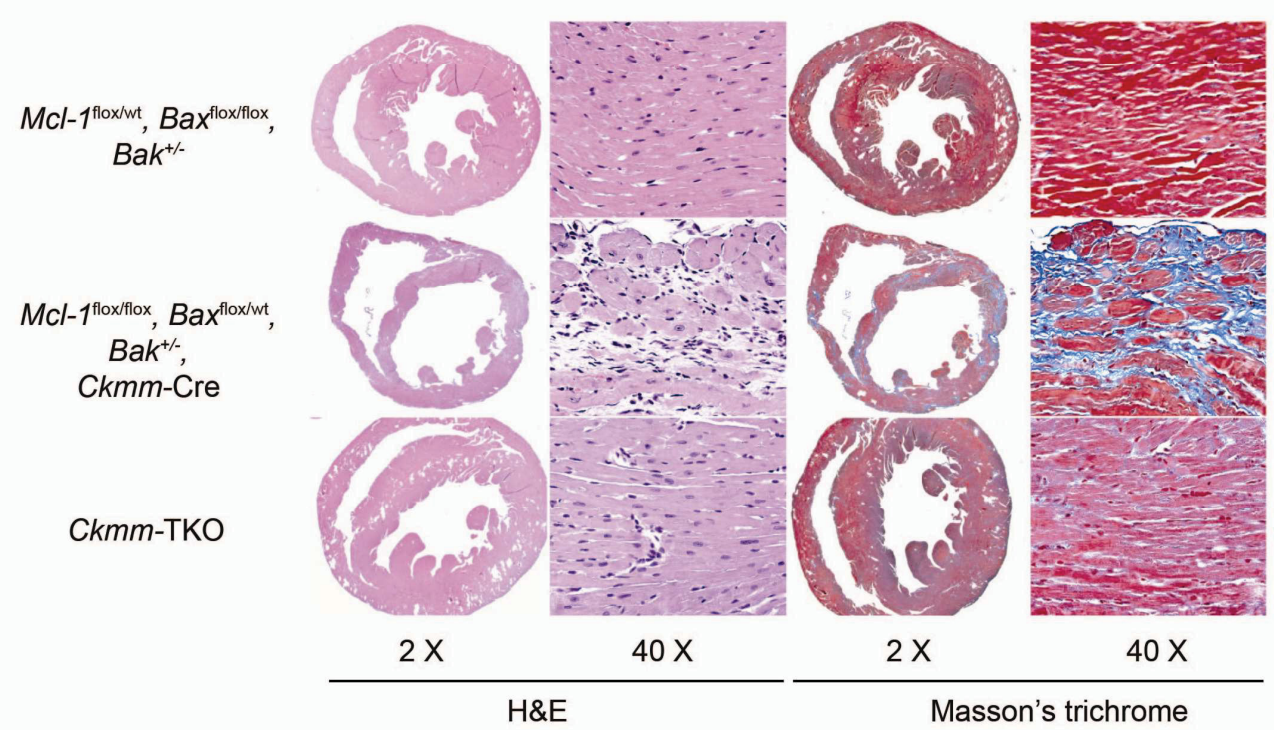

Figure 3-10. Genetic ablation of $B a x$ and $B a k$ improves cardiac function and survival of constitutive cardiac-specific $\mathrm{Mcl}-1$ deletion

(A) Survival of $\mathrm{Ckmm}$-TKO mice and littermate control mice. (**) $\mathrm{P}<0.01$ by log-rank test when compared with $\mathrm{Mcl}-1^{\text {flox/flox }}, C \mathrm{kmm}$-Cre mice. (B) Contractility of $\mathrm{Mcl}-\mathrm{I}^{\mathrm{wt} / \mathrm{wt}}$, Mcl-1 ${ }^{\text {flox/flox }} \mathrm{Bax}^{\text {flox/wt }} \mathrm{Bak}^{+/-} \mathrm{Ckmm}$-Cre, Ckmm-DKO, and Ckmm-TKO hearts. The data are presented as percent of baseline (before tamoxifen treatment). (*) $\mathrm{P}<0.05$ versus littermate control mice. Error bars represent the standard error of the mean. (C) H\&E stained cross-sections of the heart indicate dilated cardiomyopathy and a loss of myofibrils, and Masson's trichrome staining indicates fibrosis in the $\mathrm{Mcl}-\mathrm{I}^{\text {flox/flox }}$ $\mathrm{Bax}^{\text {flox/wt }} \mathrm{Bak}^{+/-} \mathrm{Ckmm}$-Cre heart. Magnifications are indicated for each panel. All hearts were harvested at P84. 
and subsequently died indicated that this mouse exhibited incomplete Bax deletion (Figure 3-10A). ECHO analysis was unable to detect significant cardiac dysfunction in the other $\mathrm{Ckmm}$-TKO mice as compared with wild-type and $\mathrm{Bax}{ }^{\text {flox/flox }}, \mathrm{Bak}^{-/}, \mathrm{Ckmm}$-Cre (hereafter referred to as $C \mathrm{kmm}$ double knockout [DKO]) littermates up to $10 \mathrm{wk}$ of age (Figure 3-11B). Surprisingly, even $\mathrm{Mcl}-1^{\text {flox/flox }}, \mathrm{Bax}^{\text {flox/flox }}, \mathrm{Bak}^{+/-}, \mathrm{Ckmm}$-Cre (expressing fewer Bax and Bak alleles) had an extended life span when compared with $M c l-1^{\text {flox/flox }}$, Ckmm-Cre but still exhibited impaired cardiac contractility and eventually succumbed (Figs. 3-10A, B). Histology of Ckmm-TKO revealed that the cardiomyopathy observed in Mcl- $1^{\text {flox/flox }}, C k m m$-Cre mice was alleviated (Figure 3-10C). In contrast, $M c l-1^{\text {flox/flox }}$, $B a x^{\text {flox/flox }}, \mathrm{Bak}^{+/-}, \mathrm{Ckmm}$-Cre mice still exhibited loss of cardiac myofibers and interstitial fibrosis (Figure 3-10C). These results indicate that the more Bax and Bak alleles remaining, the more severe the cardiac phenotype observed upon $\mathrm{Mcl}-1$ deletion, suggesting that the pro-apoptotic effectors play an important role in the pathogenesis of cardiomyopathy induced by $\mathrm{Mcl}-1$ deletion.

To test whether inhibition of apoptosis was capable of abrogating the cardiac damage due to inducible $\mathrm{Mcl}-1$ deletion in adult hearts, we generated inducible cardiacspecific $M c l-1^{\text {flox/flox }}, B a x^{\text {flox/flox }}, B a k^{-/}, M y h-C e^{\text {ER }}$ (hereafter referred to as $M y h$-TKO) mice by crossing $M c l-1^{\text {flox/flox }}, M y h-C e^{\text {ER }}$ mice with $B a x^{\text {flox/flox }}, B a k^{-/-}$mice. Immunoblot analyses indicated efficient deletion of MCL-1, BAX, and BAK in cardiac tissue of tamoxifen-treated $M y h$-TKO mice (Figure 3-11A). In contrast to the prevalent mortality observed in $M c l-1^{\text {flox/flox }}, M y h-C e^{\mathrm{ER}}$ mice 3 wk after tamoxifen initiation, $M y h$-TKO mice were able to survive for $>8 \mathrm{wk}$ after the induction of Cre, at which point they were sacrificed for analysis (Figure 3-11B). During the first week after tamoxifen treatment, $M y h$ - TKO mice exhibited a transient but significant defect in both EF and FS that eventually resolved (Figure 3-11C). Upon sacrifice, $M y h$-TKO hearts were comparable with littermate control $\mathrm{Mcl}-\mathrm{I}^{\text {flox/wt }}$ hearts without the appearance of overt apoptosis, interstitial fibrosis, and loss of myofibers observed in $M c l-1^{\text {flox/flox }}, M y h$-Cre ${ }^{\mathrm{ER}}$ hearts (Figure 3-2E, F; Figure 3-11D; Figure 3-5B, C). We also observed the induction of $\mathrm{BIM}$ and BNIP3 in the Myh-TKO hearts, indicating that they are upstream of BAX and BAK (Figure 3-6). Therefore, co-deletion of BAX, BAK, and MCL-1 prevents heart failure and overt cardiomyopathy by blocking cell death.

\section{Genetic Ablation of Bax and Bak Is Insufficient to Restore Mitochondrial Abnormalities Induced by Mcl-1-deletion}

Although ECHO and histological analyses did not display significant cardiac dysfunction, analyses of the ultrastructure of TKO hearts by TEM revealed that the myocardial organization and mitochondrial ultrastructure were quite heterogeneous among different regions of the heart (Figure 3-12). Examination indicated that both interfibrillar and subsarcolemmal mitochondria appear equally affected and that the mitochondria with abnormal ultrastructure were primarily found in the endocardium and not the epicardium. Both $C \mathrm{kmm}$-TKO and $M y h$-TKO hearts still exhibited regions of rounded mitochondria that were disorganized and dispersed (Figure 3-13). The cristae structure of the damaged $C \mathrm{kmm}$-TKO and $M y$ h-TKO mitochondria were fragmented and 


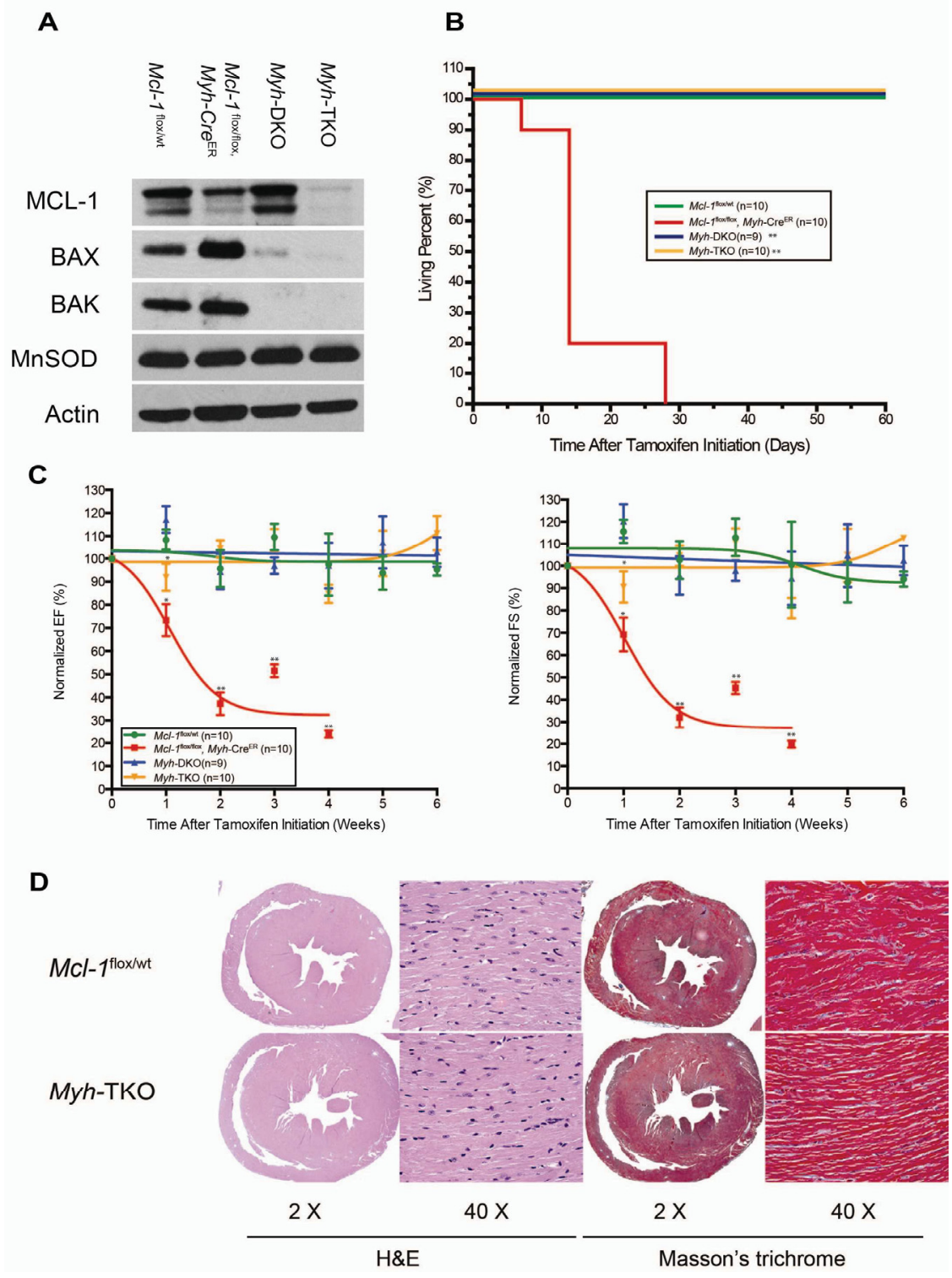

Figure 3-11. Genetic ablation of $B a x$ and $B a k$ improves cardiac function and survival of inducible cardiac-specific $\mathrm{Mcl}-1$ deletion

(A) Tamoxifen induced Mcl-1, Bax, and Bak deletion in heart. All tissues were harvested 8 wk after tamoxifen initiation and immunoblotted with MCL-1, BAX, and BAK. MnSOD served as the mitochondrial marker and Actin served as the loading control. (B) Survival of Myh-TKO mice and littermate controls. (**) $\mathrm{P}<0.01$ as determined by logrank test when compared with Mcl- 1flox/flox Myh-CreER. (C) Contractility of $\mathrm{Mcl}$ $l^{\mathrm{wt} / \mathrm{wt}}, M c l-1^{\text {flox/flox }} M y h-\mathrm{Cre}^{\mathrm{ER}}, M y h$-DKO, and $M y h$-TKO hearts. The data are presented as percent of baseline (before tamoxifen treatment). (*) $\mathrm{P}<0.05$; (**) $\mathrm{P}<0.01$. Error bars represent the standard error of the mean. (D) H\&E-stained cross-sections of the heart indicate no evidence of cardiomyopathy in the $M y h$-TKO hearts. Masson's staining indicates no evidence of fibrosis in $M y h$-TKO hearts. All hearts were harvested 8 wk after tamoxifen initiation. Magnifications are as indicated. 


\section{A}

MCl-1 1loxwt $^{\text {B }}$ Bax floxflox,

$\mathrm{Bak}^{+/-}$

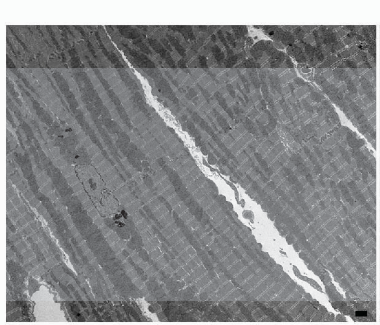

$M C l-$ flox/wt $^{-10}$

B

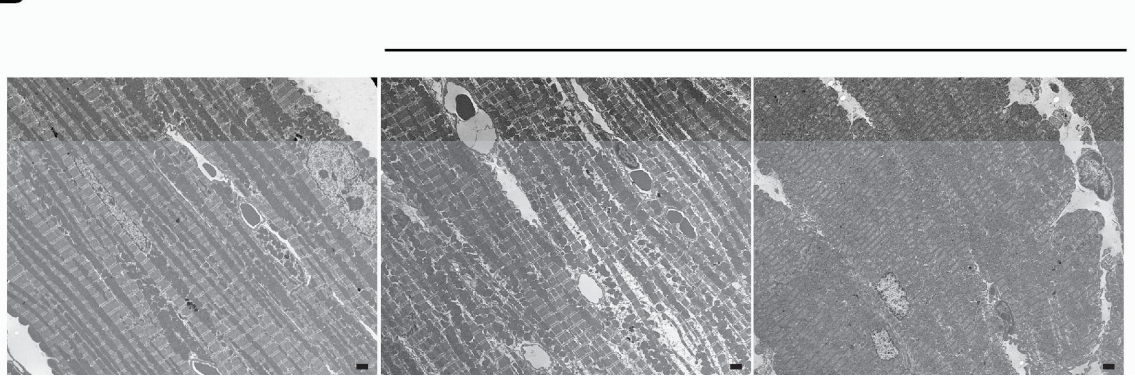

Ckmm-TKO

$\mathrm{Bak}^{+\prime}, \mathrm{Ckmm}-\mathrm{Cre}$

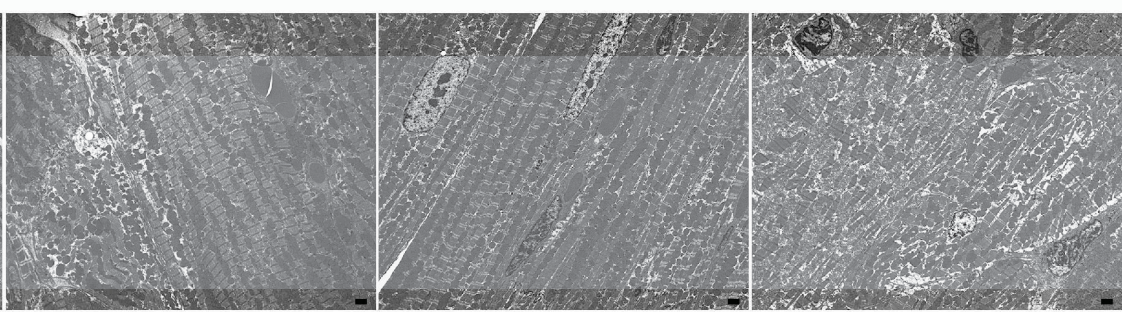

Myh-TKO

Figure 3-12. Genetic ablation of $B a x$ and $B a k$ is insufficient to obviate mitochondrial abnormalities induced by $M c l-1$-deletion

Low magnification transmission electron micrographs indicated disrupted myocardium, mitochondria with abnormal cristae structure in (A) Constitutively $\mathrm{Mcl}$-1-deleted CkmmTKO hearts (harvested at P84), (B) tamoxifen induced Mcl-1-deleted Myh-TKO hearts (eight weeks after tamoxifen initiation). The scale bars represent $2 \mu \mathrm{m}$. 


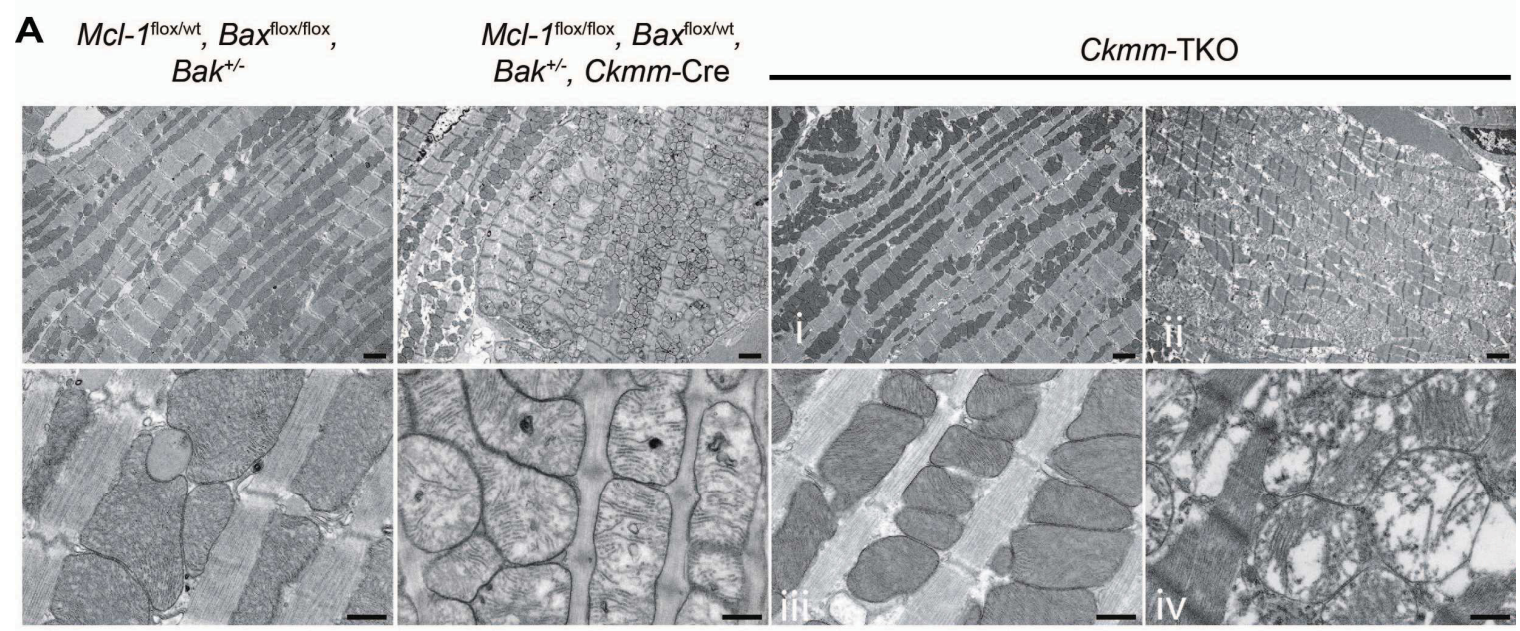

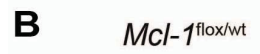

Myh-TKO

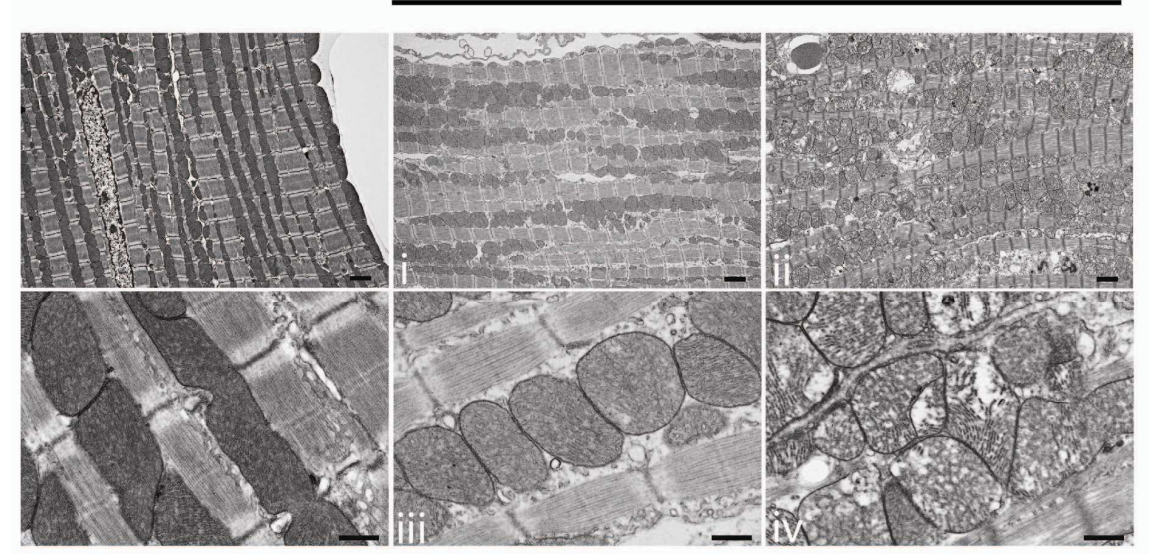

Figure 3-13. Mitochondrial ultrastructural abnormalities in $\mathrm{Mcl}$-1-deleted hearts cannot be prevented by genetic ablation of $B a x$ and $B a k$

Transmission electron micrographs indicate disrupted myocardium and disorganized mitochondria with abnormal cristae structure in (A) Constitutively $\mathrm{Mcl}$-1-deleted $\mathrm{Ckmm}$ TKO hearts (harvested at P84). (B) Tamoxifen induced $M c l$-1-deleted Myh-TKO hearts (8 wk after tamoxifen initiation). For the $C k m m$-TKO and $M y h$-TKO samples, regions with relatively normal morphology are indicated by $\mathrm{i}$ and iii, whereas the regions with abnormal ultrastructure are indicated by ii and iv. Bars: top panels (lower magnification), $2 \mathrm{~mm}$; bottom panels (higher magnification), $500 \mathrm{~nm}$. 
degraded but possessed intact OMMs (Figure 3-13). These defects were similar to those observed in the mitochondria from $M c l-1$-deleted myocardium (Figure 3-7A, B). Additionally, TEM analyses of $C \mathrm{kmm}$-TKO and Myh-TKO hearts still revealed myocardial regions that featured some loss of myofibrils, but not to the same extent as those occurring in Mcl-1-deleted hearts (Figure 3-7A, B).

Since the TEM detected that $M y h$-TKO hearts still exhibit defective mitochondria, we tested whether $M y h$-TKO-isolated mitochondria displayed detectable respiratory deficiencies. To this aim, $M y h$-TKO, $M y h$-DKO, and $M c l-1$-deleted cardiac mitochondria were isolated, and their ability to respire was determined by measuring OCR. As previously determined, mitochondria from $\mathrm{Mcl}-1$-deleted hearts displayed decreased OCR (Table 3-2). In contrast, Myh-DKO mitochondria respired similarly to control mitochondria, indicating that loss of both BAX and BAK does not result in defective mitochondrial respiration (Table 3-2). However, despite mitigating the symptoms of $\mathrm{Mcl}$ 1 deletion, the mitochondria from Myh-TKO mice still exhibited defective OCR, indicating a respiratory defect (Table 3-2). These data suggest that MCL-1 may also play a role in promoting normal cardiac mitochondrial respiration that is distinct from its ability to inhibit apoptosis.

The mitochondrial respiratory defects induced by cardiac-specific $M c l-1$ deletion alone led to the loss of mtDNA (Table 3-3). Therefore, we investigated whether the loss of mtDNA could be alleviated in the $M y h$-TKO hearts. Unlike $M y h$-DKO cardiac tissue, which expressed similar levels of mtDNA when compared with nondeleted littermate control hearts, the hearts from Myh-TKO mice still displayed the loss of mtDNA (Table 3-3). Taken together, these data indicate that even when apoptosis is genetically blocked by loss of BAX and BAK, the loss of MCL-1 expression in cardiac tissue results in mitochondrial dysfunction.

\section{Mcl-1-loss in Cardiomyocytes Impairs Autophagy Induction While Co-deletion of Mcl-1, Bax, and Bak Restores Cardiac Autophagy}

Autophagy and the ubiquitin-proteasome system are responsible to the efficient degradation of cytoplasmic proteins and dysfunctional organelles by sequestration within an autophagosome that fuses to lysosomes for degradation (Levine and Klionsky 2004). In cardiomyocytes, basal autophagy is indispensable to maintain normal cardiac function under baseline condition (Nakai et al. 2007); however, the adaptive role of autophagy under cardiac stress is still under debate dependent upon the genetic model (Nakai et al. 2007; Zhu et al. 2007). In our model, we observe that Mcl-1-deleted hearts exhibit reduced LC3II/LC3I ratios, elevated p62 levels, and deposition of ubiquitinylated aggregate, indicating an inhibition of autophagy (Figure 3-14). In contrast, the Myh-TKO hearts reveal a robust elevation of LC3II/LC3I ratios, reduced p62 levels, and diminished ubiquitin aggregates in the heart (Figure 3-14). Therefore, the loss of BAX and BAK in the context of MCL-1 deficiency restores normal induction of autophagy. 
A

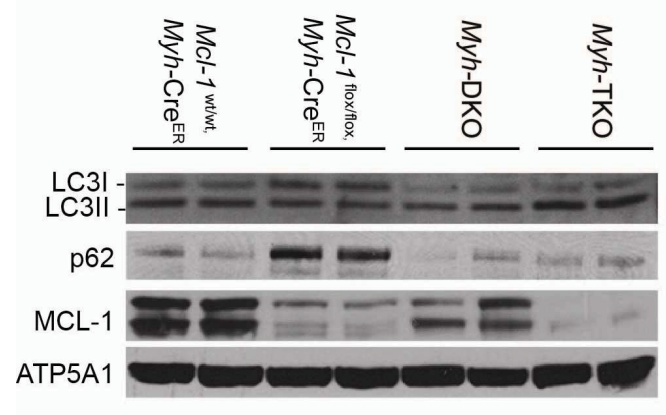

C

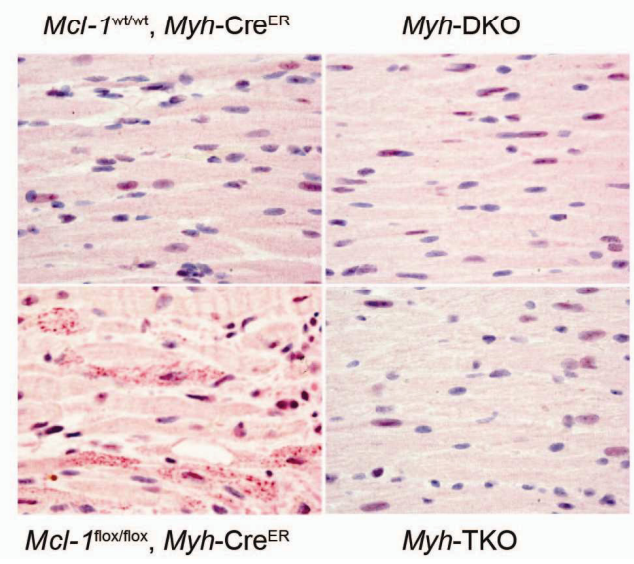

B
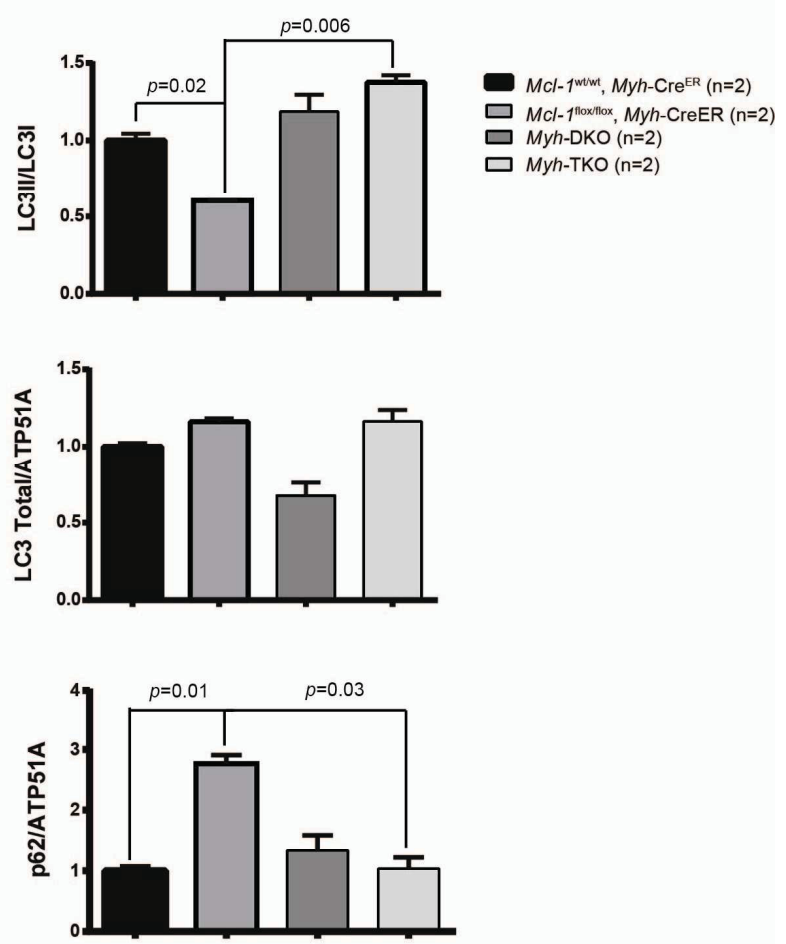

Figure 3-14. Mcl-1-loss in cardiomyocytes impairs autophagy induction while codeletion of $\mathrm{Mcl}-1, \mathrm{Bax}$, and $\mathrm{Bak}$ restores cardiac autophagy

(A) Immunoblot analyses of hearts isolated from indicated genotypes at 3 weeks after tamoxifen administration. Autophagy markers LC3II/I and p62 are shown. Efficiency of Cre-deletion is indicated by MCL-1 protein and ATP5A1 serves as loading control. Each lane represents a separate mouse. (B) Quantitation analyses of LC3II/LC3I, p62, and total LC3 levels. Error bars represent the standard error of the mean. (C) Ubiquitin immunohistochemistry from longitudinal heart sections from $\mathrm{Mcl}-1^{\mathrm{wtwt}} \mathrm{Myh}$-Cre ${ }^{\mathrm{ER}}, \mathrm{Mcl}$ $l^{\text {flox/flox }} M y h$-Cre ${ }^{\mathrm{ER}}, M y h$-DKO and $M y h$-TKO mice (3 weeks after tamoxifenadministration). Red staining in $M c l-1^{\text {flox/flox }} M y h-\mathrm{Cre}^{\mathrm{ER}}$ heart section indicates ubiquitin aggregates in cardiomyocytes. Magnification, $64 \times$. 


\section{CHAPTER 4. DISCUSSION}

\section{Role of MCL-1 in the Heart Homeostasis and Heart Disease Treatment}

Genetic ablation of MCL-1 in a myriad of cellular lineages leads to cell autonomous deficiencies, indicating that MCL-1 is a unique pro-survival BCL-2 family member (Opferman et al. 2003; Opferman et al. 2005; Arbour et al. 2008; Steimer et al. 2009). The Opferman laboratory has revealed that besides its classical anti-apoptotic aspect, MCL-1 also facilitates mitochondrial physiology in vitro, and these two distinct functions are dependent upon the sub-mitochondrial localizations of MCL-1 (Perciavalle et al. 2012). Therefore, it will be important to delineate the contributions of MCL-1's dual functions in an in vivo system. Because the heart is particularly sensitive to death stimuli and highly dependent upon mitochondrial function, it is ideal for the study of MCL-1's dual functions. Moreover, the role of MCL-1 in heart homeostasis has not been determined yet.

\section{BCL-2 Family Members and Cardiomyocyte Death}

Since cardiomyocytes lack the ability to regenerate and repair, they have developed several strategies to minimize cell death in the heart (Wencker 2003). It is reported that cardiomyocytes possess low level of APAF-1, and microinjection of cytochrome $c$ into them is insufficient to induce cell death (Potts et al. 2005). However, increased apoptotic cell death has still been implicated in various heart diseases such as myocardial infarction, ischemia/reperfusion injury, cardiomyopathy and heart failure (Narula et al. 1996; Olivetti et al. 1997; Saraste et al. 1997).

BCL-2 family members are pivotal for the regulation of cell death; therefore their roles in cardiomyocyte death have been intensively studied over the past decades. Under steady state, genetic ablation of either $B c l-2$ or $B c l-X_{L}$ revealed no overt cardiovascular abnormalities (Veis et al. 1993; Motoyama et al. 1995; Fernandez et al. 2001). However, those anti-apoptotic proteins have been revealed to be protective for cardiomyocytes under various stresses. For example, endogenous BCL-2 and BCL- $\mathrm{X}_{\mathrm{L}}$ level decline during the ischemia/reperfusion injury. In contrast, overexpression of BCL-2 or BCL-X blocks apoptosis and results in smaller infarcts, improved cardiac function and survival (Brocheriou et al. 2000; Chen et al. 2001; Imahashi et al. 2004). Moreover, transgenic expression of BCL-2 can attenuate cardiomyopathies by inhibiting apoptotic cell death (Milner et al. 2000; Weisleder et al. 2004; Maloyan et al. 2010). A variety of proapoptotic BH3-only family members, including BID, PUMA, BAD, BNIP3, and NIX have been reported to be induced upon cardiac stress and are implicated in the pathogenesis of the disease (Murriel et al. 2004; Galvez et al. 2006; Toth et al. 2006; Diwan et al. 2008). Ablation of pro-apoptotic effector Bax has been shown to be protective against ischemia/reperfusion injury (Hochhauser et al. 2003). These data indicate that while both pro- and anti-apoptotic BCL-2 family members clearly play 
important roles in modulating the response of cardiomyocyte death to various stresses, the specific genetic regulators have remained unknown.

\section{MCL-1 Is an Essential Pro-survival Regulator for Heart Homeostasis}

Our data demonstrated that cardiac-specific deletion of anti-apoptotic $\mathrm{Mcl}-1$ leads to a fatal dilated cardiomyopathy under steady state exhibiting cardiomyocytes loss, ultrastructural abnormalities in both myocardium and associated mitochondria, and impaired mitochondrial respiration. The number of apoptotic cardiomyocytes was elevated in the $\mathrm{Mcl}$-1-deleted heart. Similar outcomes were observed either when $\mathrm{Mcl}-1$ was deleted by $\mathrm{Ckmm}$-Cre initiating at E13 or when $\mathrm{Mcl}-1$ is deleted during adulthood by tamoxifen-inducible $M y h-\mathrm{CRE}^{\mathrm{ER}}$.

The development of the heart starts at E7.5, beating heart tube can be observed at E8.5, and the primary four cardiac chambers is formed by E10.5 (Xin et al. 2013). Apoptosis is a normal and necessary phenomenon for embryonic heart development as it is involved in the developmental remodeling of the organ, and has been reported to be essential for ventricular morphogenesis from E11-E16 (Abdelwahid et al. 1999). After birth, apoptosis still plays an important role in the ventricular structural remodeling of the postnatal heart (Fernandez et al. 2001). Prior to birth, fetal blood is oxygenated by placenta and enters the right ventricle. Immediately after birth, the neonatal lung expands upon the first breath and establishes pulmonary circulation, resulting in a drop in the pressure load on the right ventricle and an increase in the pressure load on the left ventricle. This mechanical change induces a proliferative response in the left ventricle, and an apoptotic response in the right ventricle, which results in the final structure of the mature and functional heart. Therefore, the excessive apoptosis in the heart induced upon Mcl-1 deletion during the embryonic stage could impair the embryonic heart development and postnatal cardiac remodeling, leading to cardiac dysfunction and heart failure.

Apoptotic cell death in neonatal heart starts to diminish at P4.5 and then maintains at a very low level throughout the adulthood (Fernandez et al. 2001). When $\mathrm{Mcl}-1$ was deleted in adult hearts, we observed that the apoptotic rate reached $2 \sim 3 \%$, which was significantly higher than wild-type heart, and sufficient to cause cardiomyopathy, heart failure and final death. After P7, the heart lost the regenerative ability and cardiomyocyte loss can only be repaired by connective tissue regeneration (Porrello et al. 2011). As a result, intensive interstitial fibrosis is observed in $\mathrm{Mcl}$-1-deleted heart.

Anti-apoptotic MCL-1 interacts with pro-apoptotic proteins to prevent the induction of cell death. It can either bind to $\mathrm{BH} 3$-only proteins to prevent them from activating $\mathrm{BAX}$ and $\mathrm{BAK}$, or directly interact with BAX and BAK, blocking the MOMP and the release of apoptogenic proteins from IMS (Thomas et al. 2013). Since Mcl-1 deletion induced cardiomyocyte apoptosis accompanied by an increase in expression of pro-apoptotic proteins, including BIM, BNIP3 and BAX; it is not surprising to observe that co-deletion of Bax and Bak, the essential intrinsic apoptotic effectors (Lindsten et al. 
2000), could substantially rescue the dilated cardiomyopathy and lethality upon $\mathrm{Mcl}-1$ deletion.

Moreover, BAX can also regulate necrotic cell death by increasing the threshold for the opening of the MPTP, which is independent of its pro-apoptotic function (Whelan et al. 2012). In the myocardial infarction model, deletion of Bax and Bak significantly reduced the infarct size, and no additional rescue effect was observed by further deletion of Cyclophilin-D over the Bax and Bak-deficient background, suggesting that BAX and Cyclophilin-D are redundant in preventing ischemia-induced necrosis. Thus, it is reasonable to postulate that co-deletion of Bax and Bak in the MCL-1-deficient background can not only block apoptosis, but also prevent necrosis. Indeed, in the Thomas et al. publication (Thomas et al. 2013), co-deletion of Cyclophilin-D, the key component of the mPTP, along with Mcl-1 in mouse hearts attenuates, but does not completely alleviate cardiac dysfunction and lethality, indicating that inhibiting necrotic death only partially rescues the cardiac dysfunction caused by $M c l-1$-deletion. In contrast, co-deletion of Mcl-1, Bax, and Bak in mouse hearts more completely rescued the lethality of the cardiac dysfunction caused by $\mathrm{Mcl}-1$-deletion, suggesting that while loss of $\mathrm{Mcl}-1$ may induce mPTP-dependent necrosis, the induction of Caspase-3 induced by $\mathrm{Mcl}-1$ deletion indicates that cytochrome $c$ is being released, thereby triggering apoptosis. Both of apoptosis and necrosis can be alleviated by deletion of Bax and Bak. Therefore, the critical role for MCL-1 in cardiomyocytes is to prevent the induction of cell death.

We also identified that deletion of Mcl-1 impairs autophagy induction in the myocardium. This was unexpected since the anti-apoptotic BCL-2 family members have been reported to interact with Beclin-1, an essential protein for autophagosome formation, to inhibit autophagy (Pattingre et al. 2005; Maiuri et al. 2007). Also, genetic ablation of Mcl-1 in the nervous system has been revealed to induce autophagy (Germain et al. 2011). Autophagy is a conserved process to clear damaged organelles and proteins, provide nutrients to stressed cells. Particularly, autophagy has been shown to be important for the heart homeostasis under steady status (Nakai et al. 2007). Therefore, the inhibition of autophagy in the Mcl-1-deleted heart may lead to the accumulation of malfunctional proteins (such as p62) and damaged organelles (such as mitochondria), which could contribute to the development of cardiac dysfunction.

The cross talk between apoptosis and autophagy has been reported. For example, Caspase-3, -7 and -8 can cleave Beclin-1 and class III phosphatidyl inositol-3-kinase (PI3KC3), two components of the autophagy-inducing complex, to inhibit autophagy. And the C-terminal cleavage product of Beclin-1 can amplify the mitochondria-mediated apoptosis in response to nutrient deprivation (Wirawan et al. 2010). Indeed, in the Thomas et al. publication, the protein level of Beclin-1 is decreased in the Mcl-1-deleted heart (Thomas et al. 2013). The autophagy-related protein, Atg5, has also been reported to be cleaved by Calpain during apoptosis, and the truncated product can interact with BCL-2 proteins to facilitate apoptosis (Yousefi et al. 2006). Since apoptosis is induced in the MCL-1-deficient heart, this could explain the observed autophagy inhibition in our model. Conversely, a previous study has demonstrated that autophagy could be induced when apoptosis is inhibited. When Bax and Bak double-knock MEFs are treated with 
apoptotic stimuli such as etoposide, they fail to undergo apoptosis but reveal induction in autophagy followed by delayed cell death, and can be alleviated by knocking-down Beclin-1 or Atg5 (DiMauro and Schon 2003). Therefore, in our model, when the intrinsic cell death pathway is blocked by co-deletion of Bax and Bak, it is possible that the mitochondrial dysfunction induced by $\mathrm{Mcl}-1$ loss produces mitochondrial stress that drives autophagy in an attempt to alleviate the damage. The mechanism for how MCL-1 regulates autophagy induction in the heart is still unclear, and will be an important topic of future research.

Interestingly, no overt defects in the skeletal muscle were observed upon CkmmCre-mediated ablation of Mcl-1. There were low levels of muscle fiber necrosis, but no gross muscular abnormalities observable even in the skeletal muscle of C $\mathrm{kmm}$-TKO mice. One possibility is that skeletal muscle, which possesses the ability to regenerate, may be able to functionally compensate for tissue damage unlike cardiac muscle (Ciciliot and Schiaffino 2010). Additionally, it is possible that the skeletal muscle will not reveal defects until the muscle is stressed. For example, exercise-induced oxidative stress has been reported to reveal myopathy phenotype in a mouse model without spontaneous skeletal muscle disorder (Powers and Jackson 2008; Rederstorff et al. 2011). Conversely, MCL-1 function in the skeletal muscle may not be as important as that of cardiac muscle due to compensation by other family members (Dominov et al. 2001; Schwartz 2008). Further work will be necessary to fully realize why skeletal muscle appears to be less affected by $M c l-1$-deletion.

\section{MCL-1 and Cardiac Mitochondria Physiology}

In the Mcl-1-deleted hearts, we observed massive damage in mitochondrial cristae structure, which is quite similar to what has been revealed in the MCL-1-deficient SV40 transformed MEFs and liver (Perciavalle et al. 2012). Mcl-1-deleted hearts possess less coupled mitochondria with impaired respiration, indicating decreased ATP production since mitochondrial respiration is coupled with ADP phosphorylation. In addition, the heart is among the most mitochondrial-rich organs, and mitochondrial-generated ROS is the major factor in oxidative stress to the heart (Dorn and Scorrano 2010). Dysfunctional mitochondria can cause elevated production of ROS and ROS can cause mtDNA damage and inactivate iron-sulfur center containing enzymes on ETC (Esposito et al. 1999). Consistently, cardiac-specific deletion of $\mathrm{Mcl}-1$ leads to decreased mtDNA content, and reduced enzymatic activity of ETC complex I and IV, but not complex II. This kind of change is usually observed in cases of respiratory dysfunction due to mtDNA defects, because complex II activity is solely dependent upon nuclear genome, while subunits of complex I and complex IV are encoded by mitochondrial genome (DiMauro and Schon 2003; Chen et al. 2010). Therefore, MCL-1 is necessary for the maintenance of mitochondrial morphology and physiology.

Since we deleted full-length MCL-1 in our mouse model, it could be possible that the observed mitochondrial dysfunction is secondary to the primary cell death due to ablation of the anti-apoptotic MCL-1. Co-deletion of Bax and Bak in the MCL-1- 
deficient background enables us to rule out this possibility and only observe the mitochondrial aspect of MCL-1. Despite grossly rescuing cardiac function and mouse survival, mice lacking Mcl-1, Bax, and Bak in the heart still exhibit cardiac lesions and defects in mitochondrial function, as evidenced by deficient mitochondrial respiration and the loss of mtDNA. These data suggest that MCL-1 plays an important role beyond simply inhibiting cell death in the heart. Because cardiac function is highly dependent upon mitochondrial bioenergetics, MCL-1 may be important for the long-term cardiac homeostasis under both steady and stressed states. Further study on the TKO mice would be necessary to reveal the cardiac phenotypes in response to stress.

We have demonstrated in model cell lines and liver mitochondria that different forms of MCL-1 reside in distinct mitochondrial localizations where they exhibit separable functions (Perciavalle et al. 2012). Briefly, the OMM-localized MCL-1 possesses pro-survival function by interacting with pro-apoptotic proteins to prevent cell death. In contrast, the matrix-localized MCL-1 does not inhibit cell death, but instead promotes mitochondrial physiology. The matrix-localized MCL-1 facilitates mitochondrial cristae structure to maintain normal mitochondrial morphology, IMM fusion, mitochondrial OXPHOS, and mitochondrial supercomplexes assembly. While the separation of these roles has been observed in vitro, they have not yet been shown to be relevant in vivo. In the heart, MCL-1 is present both on the OMM and within the mitochondrial matrix (Figure 4-1). Therefore it is possible that in cardiomyocytes that both localizations of MCL-1 may be necessary for maintaining cardiac function. Further work will be necessary to functionally reconstitute cardiomyocytes of $\mathrm{Mcl}$-1-deleted mice with individual MCL-1 mutants to test functionally the contribution of MCL-1's antiapoptotic function and its ability to promote mitochondrial function.

\section{MCL-1 in Therapy of Heart Disease}

Our observations indicate that MCL-1 may promote cardiac function by both inhibiting cell death and maintain mitochondrial morphology and respiration. Other prosurvival BCL-2 family members, such as BCL-2 and BCL- $\mathrm{X}_{\mathrm{L}}$, have been shown to be protective to the heart mainly in response to stresses, whereas MCL-1 is essential for the survival of the cardiomyocytes at steady state. Therefore, MCL-1 is a promising target for cardiac disease treatment and we would predict that strategies aimed at enhancing or maintaining MCL-1 function in cardiomyocytes may form the basis to improve heart function after acute and chronic cardiac stress or damage. The development of gene transfer vectors and delivery systems give us the tools to specifically express MCL-1 in cardiomyocytes to enhance its function. Viral vectors, such as retroviruses, adenoviruses, and adeno-associated viruses, are commonly used to transfer genes to cardiomyocytes (Wolfram and Donahue 2013). 


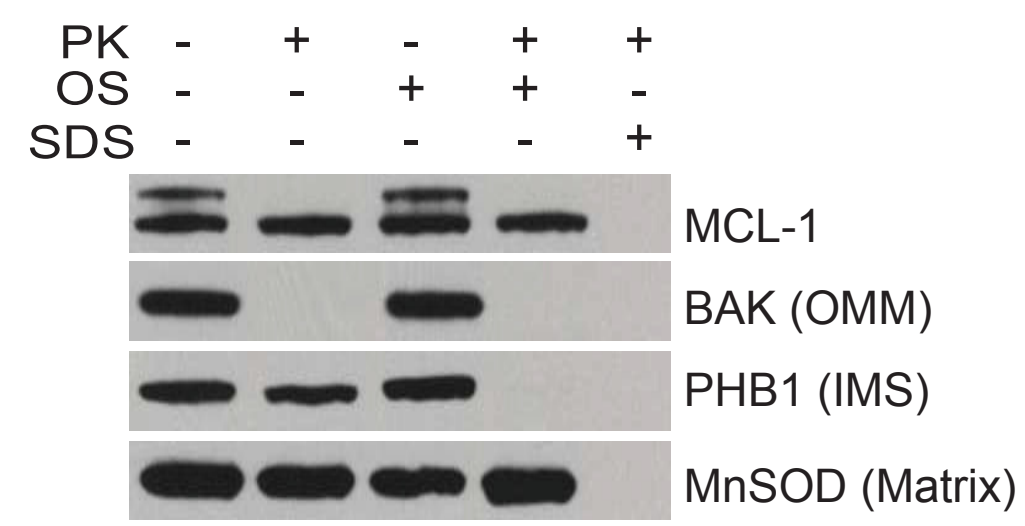

Figure 4-1. MCL-1 resides in different submitochondrial localizations in the heart The heavy membrane (HM) fraction is isolated from heart. Proteinase K (PK) digests exposed proteins, osmotic shock (OS) disrupts OMM, and detergent (SDS) solubilizes both inner and outer mitochondrial membranes. Lysates are resolved and subjected to immunoblot analysis. Markers for OMM, inner membrane space (IMS), and matrix are indicated. 


\section{MCL-1 as a Target for Cancer Therapy}

Malignant cells need to acquire resistance to mitochondrial-mediated apoptosis and undergo metabolic alteration to support the initiation and progression of cancer (Kroemer 2006). MCL-1 is a unique BCL-2 family member as it can block cell death and promote mitochondrial physiology. Moreover, MCL-1 is one of the most amplified genes found in 26 human cancers (Beroukhim et al. 2010). Its overexpression is often associated with chemotherapeutic resistance in many different cancers including ALL, AML, CML, CLL, and multiple myeloma (Kaufmann et al. 1998; Wei et al. 2006; Pepper et al. 2008). Therefore, MCL-1 is an attractive target for cancer treatment.

\section{Targeting MCL-1 as a Pro-survival Protein}

Apoptosis is essential for development and homeostasis maintenance; however, dysregulation of apoptosis can lead to variety of pathologies including cancer. Cancer cells must overcome apoptotic stresses either by inducing anti-apoptotic molecules or by reducing the pro-apoptotic factors (Hanahan and Weinberg 2011). In the intrinsic apoptosis pathway, death signals trigger the activation of pro-apoptotic BH3-only BCL-2 family members, inducing the activation of pro-apoptotic effectors BAX and BAK, facilitating the release of apoptosis inducing proteins to activate caspases cascade (Opferman 2008). Anti-apoptotic BCL-2 family members, including BCL-2, BCL-XL, BCL-w, MCL-1 and BFL-1/A1, interact with pro-apoptotic BCL-2 family members to block cell death, and are often upregulated in malignant cells as a mechanism for tumor initiation, progression and therapeutic resistance. As a result, there has been an increasing interest in developing anti-apoptotic BCL-2 family inhibitors as a strategy for cancer therapy.

ABT-737/263 (navitoclax), a promising BH3 mimetic anticancer drug, has high

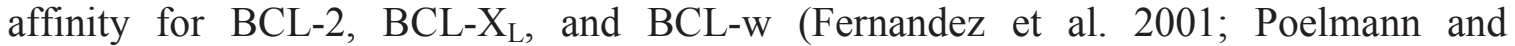
Gittenberger-de Groot 2005; Xin et al. 2013), and has been shown to cause the regression of some tumors in mouse xenograft model (Oltersdorf et al. 2005). However, its efficacy as a single agent is limited for MCL-1 overexpressed tumors. Strategies using cyclindependent inhibitors or tyrosine-kinase inhibitors to reduce MCL-1 levels through inhibition of its transcription or translation have been demonstrated to be able to sensitize resistant cancer cells to apoptotic cell death (Konopleva et al. 2006; van Delft et al. 2006; Koss et al. 2013). These kinase inhibitors may have global effects for cells and tissues, which could lead to side effects to render their systemic usage. Therefore, developing MCL-1 specific inhibitors is a more attractive therapeutic strategy and has been intensively explored in clinical researches.

\section{Targeting MCL-1 in Mitochondrial Physiology}

The role of MCL-1 in promoting mitochondrial physiology provides another clue

for targeting MCL-1 in cancer therapy. Cancer cells constitutively upregulate aerobic 
glycolysis. This phenomenon was described by Nobel laureate Otto Heinrich Warburg in 1920s and termed as the "Warburg effect" thereafter (Otto Warburg 1927). This high rate of glucose uptake is the basis for using ${ }^{18} \mathrm{~F}$-2-deoxyglucose (FDG) to differentiate between normal and solid tumor tissue in positron emission tomography (Lin 2011). In fact, the glucose transporters are often found overexpressed in cancer cells since transport of glucose across the plasma membrane is the first rate-limiting step of glucose metabolism (Macheda et al. 2005). In cancer cells, increased glucose uptake is usually accompanied by a decreased mitochondrial OXPHOS and biogenesis (Scatena 2012) due to the accumulation of defects in mitochondrial genome. The mtDNA mutations for ETC complex I, III, IV, and V have been reported in a variety of cancers and shown to be related to neoplastic transformation (Brandon et al. 2006). In addition, mutations in nDNA-encoding mitochondrial enzymes, including succinate dehydrogenase, fumarate dehydrogenase, and isocitrate dehydrogenase, have also been reported in various malignant diseases (Ward et al. 2010; Bardella et al. 2011; Picaud et al. 2011). These mutations either inhibit oxidative phosphorylation, increase the production of ROS as a mitogen and promote tumor cell proliferation, or permit tumors to adapt to new microenvironments, especially during tumor progression and metastasis (Brandon et al. 2006). Accordingly, inhibition of mitochondrial function of MCL-1 may be beneficial for the malignant progression and thus detrimental to the cancer therapy.

However, there is evidence that cancer cells still require functional mitochondria. Cancer cells with depleted mtDNA by growing in ethidium bromide show reduced growth rate, colony formation on soft agar and tumor formation in nude mice (Morais et al. 1994; Cavalli et al. 1997; Magda et al. 2008; Weinberg et al. 2010). Overexpression of oncogenic Myc has been observed in many aggressive tumors and found to upregulate mitochondrial respiration and biogenesis by the induction of TFAM and subunits of ETC complex I, uncoupling proteins, and mitochondrial membrane proteins (Li et al. 2005). Myc-mediated upregulation of mitochondrial function is also associated with induction of glutaminase, converting glutamine to glutamate, and glutamate is converted to $\alpha$ ketoglutarate to enter the TCA cycle, rendering glutamine-dependent cancer cells with active mitochondrial function (Wise et al. 2008). Recently, reduced glycolysis and increased oxidative phosphorylation in certain melanomas has been reported (Haq et al. 2013; Vazquez et al. 2013), indicating that not all cancer cells are inherently glycolytic, but rather have metabolic plasticity. Moreover, studies about the energy budget of various cancer cells under normoxic conditions demonstrate that most of the ATP in cancer cells still comes from mitochondrial oxidative phosphorylation, similar to normal cells ( $\mathrm{Zu}$ and Guppy 2004). Consequently, inhibition of the mitochondrial function of MCL-1 may lead to effective cancer therapy.

Different cancer cells may undergo different bioenergetics adjustments, either becoming more glycolytic or more oxidative, depending upon the tissue context, and stage of the cells during transformation. Therefore, targeting mitochondrial function of MCL-1 could give rise to different outcomes in cancer therapy and need further elucidation. 


\section{Potential Cardiac Complications of Targeting MCL-1 in Cancer Therapy}

Cancer treatments include chemotherapy, radiotherapy, surgical intervention and combination of different options. Many of these treatments have potential adverse side effect on cardiac function and would significantly affect the life quality, short-term and long-term survival of the patients. Common cardiac complications in cancer therapy include heart failure, myocardial ischemia, hypertension, thromboembolism, QT prolongation, and bradycardia.

Several therapies for cancer have been associated with the development of left ventricle dysfunction and heart failure. The incidence of cardiac toxicity is determined by multiple factors, such as the cumulative dose, the administration schedule, and the use of other cardiotoxic treatments (Yeh and Bickford 2009). For example, anthracylcine (such as doxorubicin)-induced left ventricle dysfunction or heart failure occurs in $3 \%$ to $26 \%$ of patients (Von Hoff et al. 1979; Swain et al. 2003). Oxidative stress is the main explanation for antracycline-induced cardiotoxicity. Other mechanisms have also been postulated, such as induction of apoptosis, decreased ATP production by cardiomyocytes, downregulation of GPx acitivity, mitochondrial respiratory defects due to mtDNA damage, and topoisomerase II beta mediated DNA double-strand breaks (Wouters et al. 2005; Lyu et al. 2007). The incidence of heart failure reported with tyrosine kinase inhibitor (such as bevacizumab, dasatanib, and imatinib) therapy ranges from $2 \%$ to $4 \%$. The mechanisms of heart failure associated with tyrosine kinase inhibitors may be related to high blood pressure, inhibition of vascular endothelial growth factor (VEGF)/VEGF receptor signaling, and inhibition of c-Abl (Miller et al. 2005; Kerkela et al. 2006; Miller et al. 2007; Cella et al. 2011). In addition, ischemia and high blood pressure are common adverse effects occurring in patients treated with tyrosine kinase inhibitors (Yang et al. 2003; Chu et al. 2007; Escudier et al. 2007; Procopio et al. 2007; Burstein et al. 2008). Therefore, cardiotoxicity related to cancer treatment is important to be recognized as it may affect the prognosis and survival of cancer patients.

Importantly, our data demonstrate that MCL-1 is an essential regulator for cardiac homeostasis, via inhibiting cardiomyocyte death and promoting cardiac mitochondrial respiration. Our observation has important implication to human health, which suggests that a potential adverse side effect associated with pharmacological MCL-1 inhibition in cancer therapy might be cardiotoxicity. Therefore, it is important to monitor the heart function of patients who are treated with MCL-1 inhibitors. Measuring systolic function through evaluation of left ventricle ejection fraction with ECHO is one of the most common measurements in monitoring and diagnosing chemotherapy-induced cardiomyopathy (Ganz et al. 1996). Endocardial biopsy is the gold standard for diagnosis, but the invasiveness of the procedure limits its use (Ganz et al. 1996). Moreover, biochemical markers, such as troponin and B-type natriuretic peptide can indicate myocardial injury before the changes in ejection fraction are apparent (Dao et al. 2001; Cardinale et al. 2002; Cardinale et al. 2004). To prevent the incidence of MCL-1 inhibition-induced cardiotoxicity, clinicians may need to use cardio protectants, minimize patients' lift-time does, as well as limit the use of other cardiotoxic treatments during cancer therapy. 


\section{CHAPTER 5. FUTURE DIRECTIONS}

\section{Contributions of Different MCL-1 Isoforms to Cardiac Function in vivo}

Previous work from our laboratory has demonstrated that different forms of MCL-1 exhibit distinct submitochondrial localizations and possess separable functions (Perciavalle et al. 2012). On the OMM, full-length MCL-1 interacts with pro-apoptotic BCL-2 family proteins to antagonize cell death, whereas the N-terminal truncated MCL-1 in the mitochondrial matrix promotes normal mitochondrial morphology and physiology (Perciavalle et al. 2012). However, the biological relevance of those separable roles has not been revealed in vivo. Our data from the cardiac-specific $\mathrm{Mcl}$-1-deleted mice indicate the possibility that both functions of MCL-1 are required for cardiac homeostasis. Therefore, it is necessary to functionally reconstitute $\mathrm{Mcl}$-1-deleted heart with individual MCL-1 isoforms to determine the contributions of MCL-1's anti-apoptotic function and its ability to promote mitochondrial physiology to cardiac homeostasis.

\section{Reconstitution of $M c l-1$-deleted Hearts by Adeno-associated Virus Delivery}

Adeno-associated virus (AAV) is a small, noneveloped DNA virus that belongs to the parvovirus family, which is very popular in gene therapy as it is able to mediate stable and efficient gene expression (Michelfelder and Trepel 2009). To date, 12 AAV serotypes have been isolated from human or primate tissues (Michelfelder and Trepel 2009), and different serotypes of AAV show various tissue preferences. Several studies have demonstrated that AAV serotype 8 (AAV8) is able to efficiently deliver genes to heart and muscle, and the expression can be sustained for months. Moreover, AAV8 infection does not affect cardiac function (Wang et al. 2005; Zincarelli et al. 2008).

To ensure AAV8-CMV is able to drive gene expression in the heart in our mouse model, newborn wild-type mice were injected with an AAV8-CMV construct expressing GFP $\left(3 \times 10^{11}\right.$ genomic copy [g.c.] per pup) through the temporal vein. Robust and stable cardiac GFP expression was observed up to 20 wks post-injection by immunoblot (Figure 5-1A). AAV8 constructs expressing different isoforms of MCL-1 with an internal FLAG-epitope tag (FLINT) to differentiate them from endogenous protein were then given to neonatal mice and $\mathrm{ECHO}$ analysis during their adulthood revealed no deleterious effect on cardiac function, indicating that ectopic expression different forms of MCL-1 was tolerated (Figure 5-1B).

The mutants used for MCL-1 reconstitution include $\mathrm{Mcl}-1^{\text {Flint }}$ (protein localizes on both OMM and IMM and functions as wild-type MCL-1), $C S^{\text {Flint }}$ (protein localizes on the OMM and retains the anti-apoptotic activity) and Matrix ${ }^{\text {Flint }}$ (protein localizes in the matrix and only promotes mitochondrial physiology). Postnatal day one (P1) Mcl- ${ }^{\text {flox/flox }}$, $M y h$-Cre neonates were injected with different transgenic constructs $\left(3 \times 10^{11}\right.$ g.c./pup $)$ or vehicle (GFP) through the temporal vein. Tamoxifen was given six weeks after birth (injection). ECHO was initiated one week after tamoxifen induction for six weeks. 

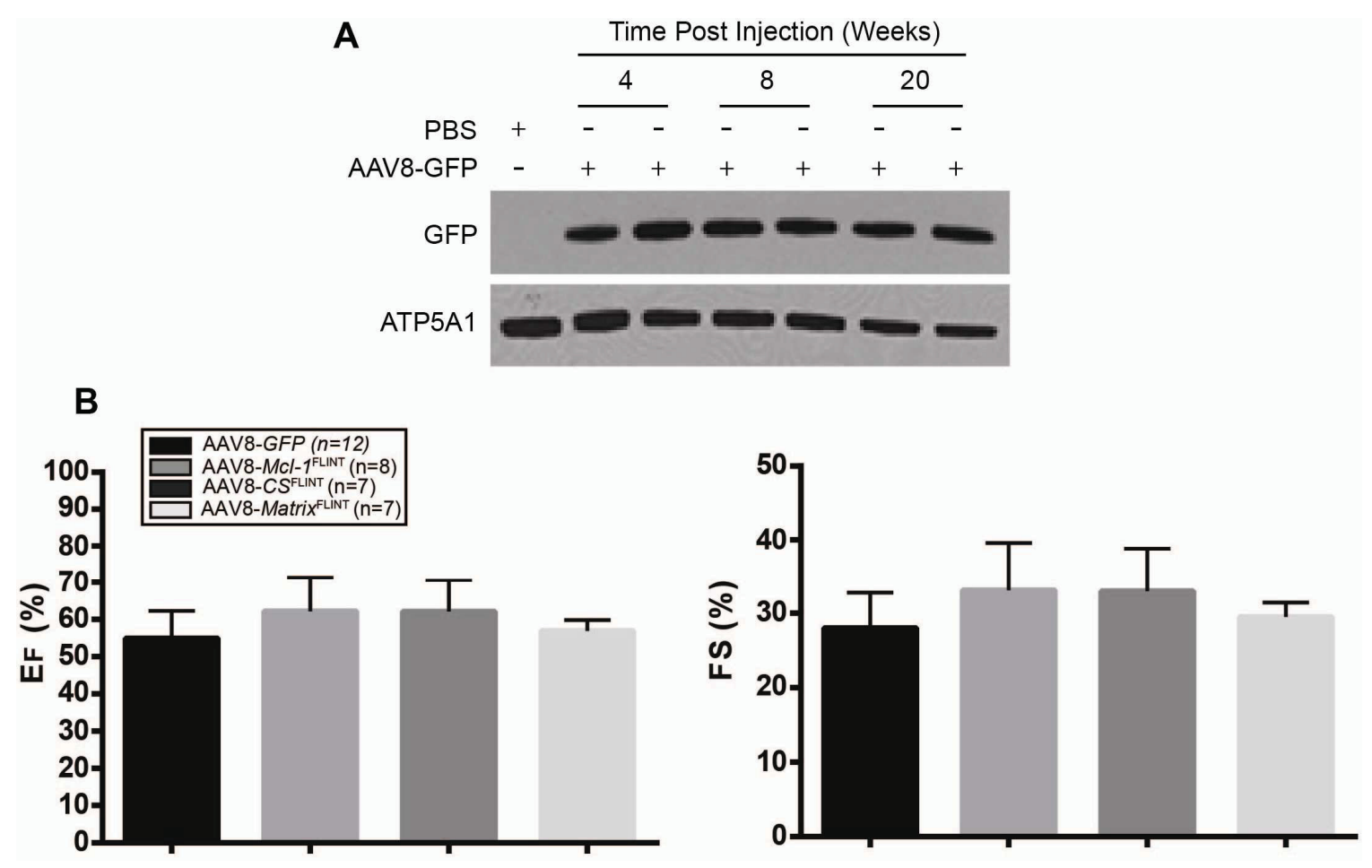

Figure 5-1. Delivery AAV constructs to the heart

(A) Immunoblot of GFP expression at indicated time points after treatment of wild-type mice with PBS (control) or AAV8-GFP. Samples were harvested at 4, 8, and 20 weeks after infection and blotted for GFP (AAV-encoded) or ATP5A1 (mitochondrial marker). (B) Ectopic expression of GFP or different forms of MCL-1 has no effect on cardiac function indicated by ejection fraction (EF) and fraction shortening (FS). Data are acquired six weeks after virus injection. The number of mice analyzed is indicated as (n). 
Deletion of endogenous $\mathrm{Mcl}-\mathrm{l}$ in GFP-expressing mice led to a fatal, dilated cardiomyopathy (Figure 5-2), similar to what has been observed previously. In contrast, expression of ectopic MCL- $1^{\text {FLINT }}$ rescued the $M c l$-1-deleted mice and improved their cardiac function (Figure 5-2). However, overexpression of either CS $^{\text {FLINT }}$ or Matrix ${ }^{\text {FLINT }}$ only partially rescued the survival, and the pathological and TEM analysis still demonstrated featured phenotypes analogue to $M c l$-1-deleted heart (Figure 5-2). Interestingly, the onset of contractile dysfunction was different in mice expressing $\mathrm{CS}^{\mathrm{FLINT}}$ and Matrix ${ }^{\mathrm{FLINT}}$. The contractility of $\mathrm{CS}^{\mathrm{FLLNT}}$ overexpressed mice were quite stable till four weeks after tamoxifen initiation and decreased drastically thereafter, whereas the contractility of Matrix ${ }^{\text {FLINT }}$ overexpressed mice started to decrease at the very beginning and the curves mostly overlap with GFP overexpressed mice. This observation indicates that anti-apoptotic function of MCL-1 is more critical for protecting cardiomyocytes against acute death while the mitochondrial function of MCL-1 is more important for keeping long-term cardiac health. Therefore, it is possible that both functions of MCL-1 are necessary for maintaining cardiac homeostasis.

Although ectopic MCL-1 ${ }^{\text {FLINT }}$ rescued the survival and cardiac function of the Mcl-1-deleted mice, myofibril, connective tissue regeneration and mitochondrial damage can still be observed in the heart (Figure 5-2C, D). The possible explanation could be not all the Mcl-1-deleted cardiomyocytes are transduced by AAV, thus they cannot be rescued and still reveal the lesions.

To resolve the caveat, we propose generating picornavirus $2 \mathrm{~A}$ peptide-linked muticstronic AAV constructs to deliver both tamoxifen-inducible Cre and individual forms of MCL-1 to the heart. Besides the previously used mutants, we include two more transgenes: combination of Matrix ${ }^{\text {FLINT }}$ and $\mathrm{CS}^{\mathrm{HIS}}$ (cleavage site mutant with a polyhistidine tag) functions as wild type MCL-1, and human BCL-w works as an antiapoptotic control (Figure 5-3). The picornavirus 2A peptides function as cis-acting hydrolase elements, mediating the "cleavage" between two proteins via ribosomal skipping (de Felipe and Ryan 2004). The conventional approaches for co-expression of multiple proteins have several limitations, such as imbalanced protein expression and large size. In contrast, the small $2 \mathrm{~A}$ peptide sequences allow for efficient and stoichiometric production of discrete proteins (Szymczak-Workman et al. 2012). To ensure the deletion of endogenous MCL-1 is cardiac specific, the cardiac-specific $\alpha$ myosin heavy chain $(\alpha-M h c)$ promoter will be used instead of the ubiquitous $C M V$ promoter (Pacak et al. 2008). And it is necessary to validate whether the $\alpha-M h c$ promoter-driven tamoxifen-inducible Cre is able to recapitulate the similar phenotypes observed in Mcl-1-deleted mice. If this is the case, after virus delivery and tamoxifen treatment, transduced cardiomyocytes should have their endogenous MCL-1 deleted and ectopic MCL-1 mutants overexpressed, alleviating the concern. 
Figure 5-2. Normal cardiac function needs both isoforms of MCL-1

(A) Contractility of $M c l-1^{\text {flox/flox }} M y h$-Cre ${ }^{\text {ER }}$ hearts overexpressed with GFP (control) or MCL-1 mutants . (*) $P<0.05$. Error bars represent the standard error of the mean. Mice were injected with AAV virus at postnatal day 1 (P1) through the temporal vein and then treat with tamoxifen six weeks after injection. (B) H\&E-stained cross sections of the heart indicate dilated cardiomyopathy and a loss of myofibrils. Fibrosis in the crosssections of the heart stained blue with the Masson's trichrome stain. (C) Transmission electron micrographs indicate disrupted myocardium and disorganized mitochondria with abnormal cristae structure in MCL-1 mutants overexpressed $M c l-1^{\text {flox/flox }}, M y h-C r e^{\text {ER }}$ hearts after tamoxifen treatment. For the $M c l-1^{\text {FLINT }}$ overexpressed samples, regions with relatively normal morphology are indicated by $\mathrm{i}$ and iii, whereas the regions with abnormal ultrastructure are indicated by ii and iv. Bars: top panels (lower magnification), $2 \mu \mathrm{m}$; bottom panels (higher magnification), $500 \mathrm{~nm}$. (D) Survival of $M c l-1^{\text {flox/flox }}, M y h$ $\mathrm{Cre}^{\mathrm{ER}}$ mice overexpressed with MCL-1 mutants after tamoxifen treatment. (E) The expression of MCL-1 mutants in the $M c l-1^{\text {flox/flox }}, M y h-\mathrm{Cre}^{\mathrm{ER}}$ hearts after AAV injection. Actin serves as the loading control. 

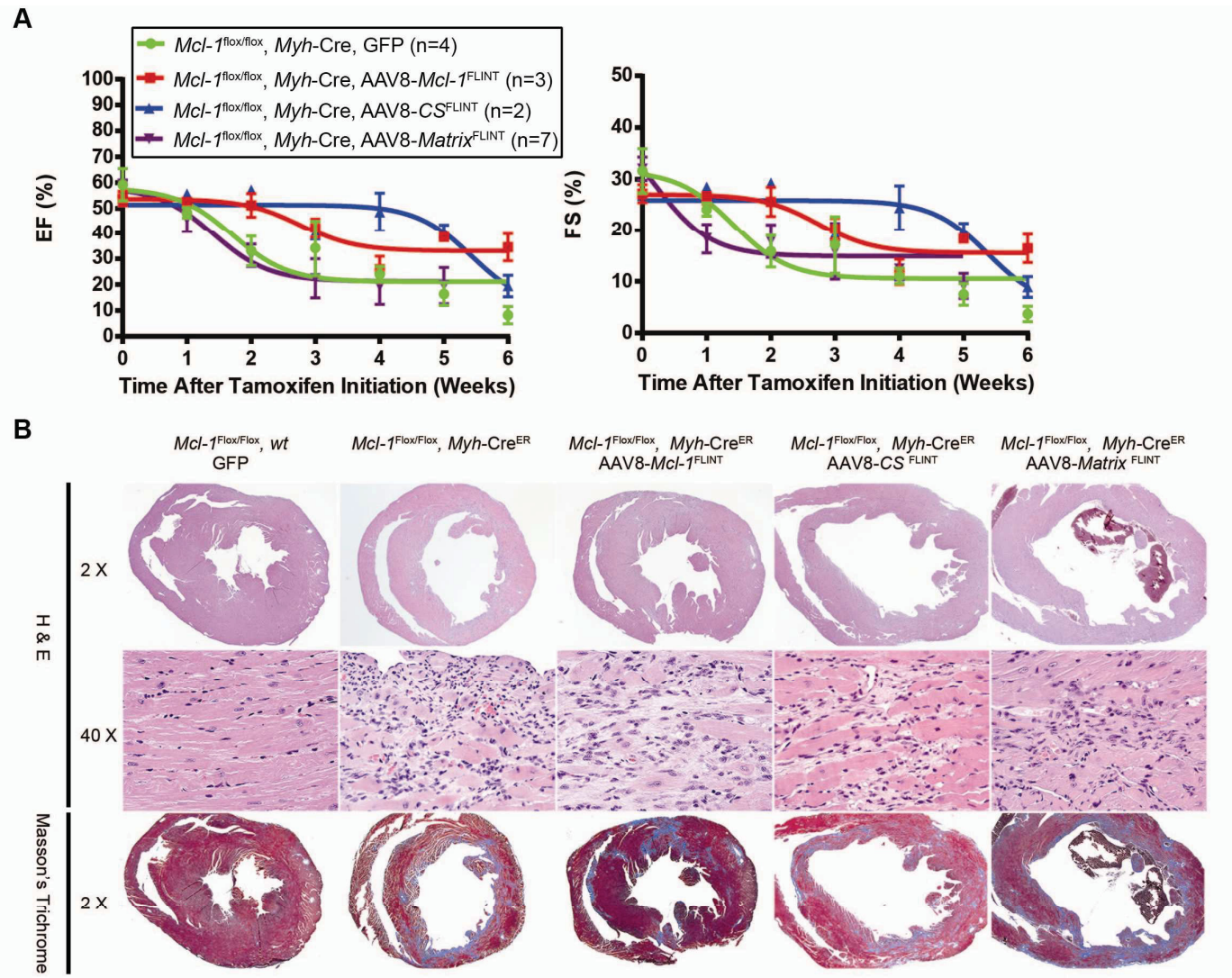

C
MCl-1 Fioxflox, wt
MCl-1 F Foxflox, Myh-Cre ER AAV8-MCl-1 FLNT $^{\mathrm{L}}$
Mcl-1Flox/Flox, Myh-Cre ER $M c l-1$ Flox/Flox, Myh-Cre ER AAV8-CS FLINT AAV8-Matrix FLIN

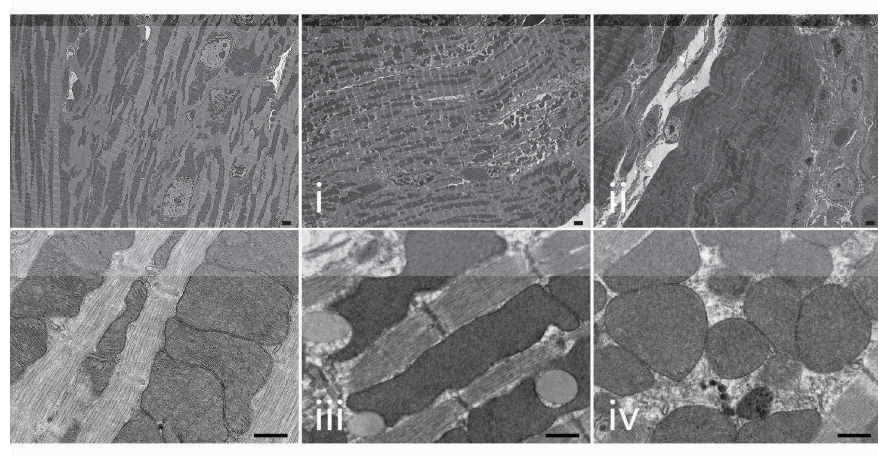

D

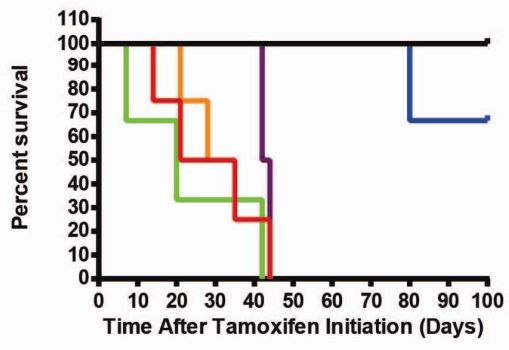

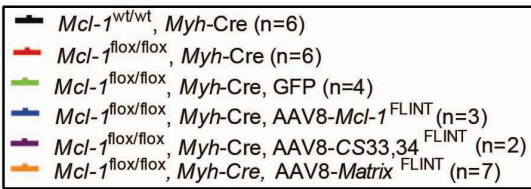

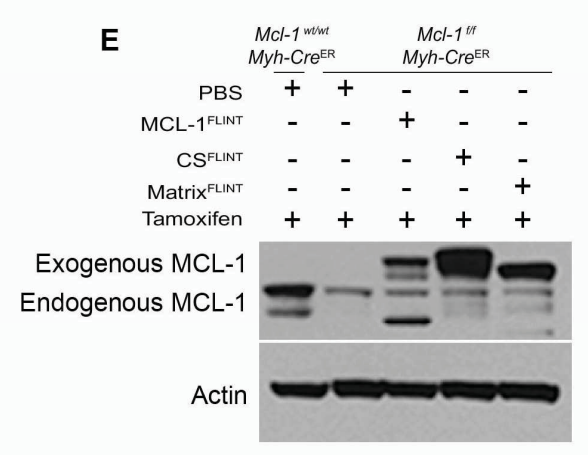




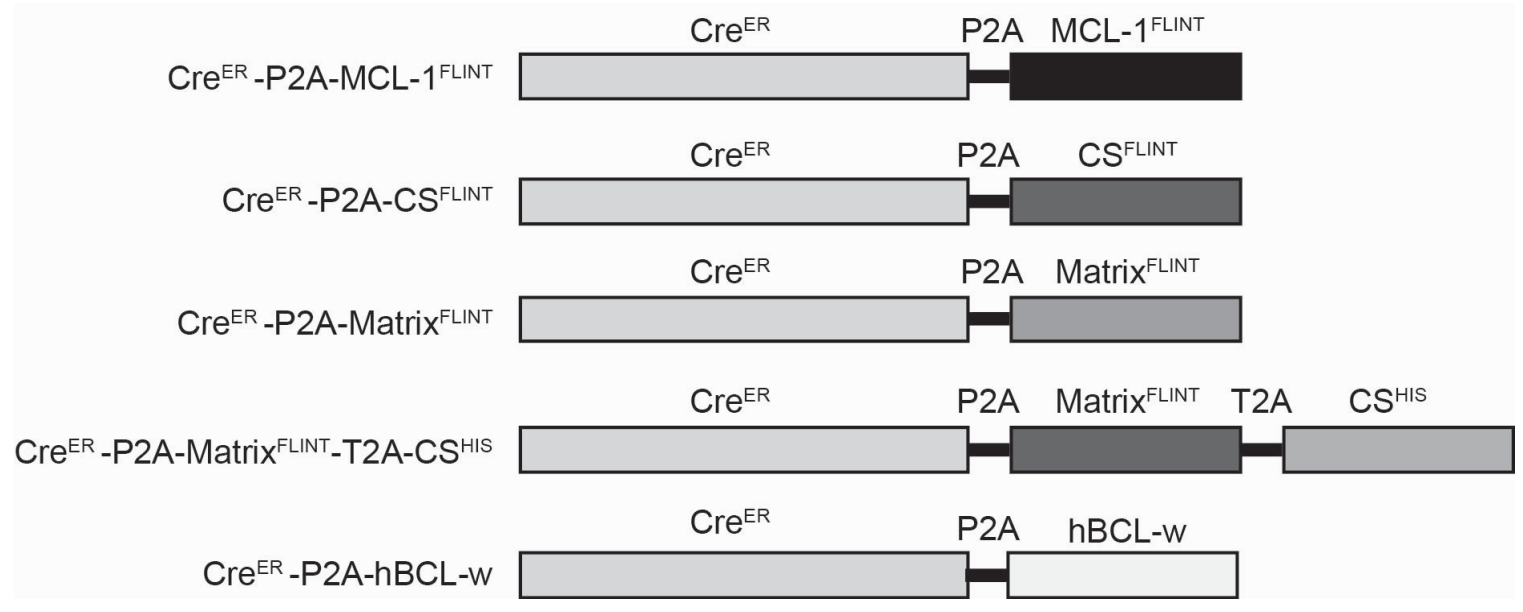

Figure 5-3. Schematic illustration of $2 \mathrm{~A}$ peptide-linked multicistronic cassettes MCL- ${ }^{\text {Flint }}$ localizes on both OMM and IMM and functions as wild-type MCL-1. CS ${ }^{\text {Flint }}$ localizes on the OMM and retain the anti-apoptotic ability. Matrix ${ }^{\text {Flint }}$ localizes in the matrix and only promotes mitochondrial physiology. Combination of Matrix ${ }^{\text {FLINT }}$ and $\mathrm{CS}^{\mathrm{HIS}}$ functions as wild type MCL-1. Human BCL-w works as an anti-apoptotic control. 


\section{Cardiac Function of TKO Mice under Stress}

Although inhibition of the intrinsic apoptotic pathway by genetic ablation of Bax and Bak significantly rescued the cardiac dysfunction due to $\mathrm{Mcl}-1$ deletion, $\mathrm{ckmm}$-TKO and tamoxifen-treated $M y h$-TKO mice still revealed defective myocardial organization and mitochondrial morphology under TEM. Furthermore, mitochondria isolated from $M y h$-TKO mice still exhibited impaired respiration and decreased mtDNA content. These data indicate that besides inhibiting apoptosis, MCL-1 is also necessary for maintaining cardiac mitochondrial function and important for long-term heart health and cardiac function under stress.

Isoproterenol is a $\beta$-adrenergic agonist and has positive inotropic and chronotropic effects on the heart, increasing the energy demands of the myocardium. Therefore, isoproterenol administration to the TKO mice may reveal more robust cardiac dysfunction since enhanced mitochondrial function is required in response to the drug. $M y h$-TKO mice were treated with tamoxifen for five days and followed by a low dose of isoproterenol (7.5 mg/kg body weight/day) for seven days (Nakai et al. 2007). The ECHO was performed one week after the initiation of isoproterenol (two weeks after tamoxifen initiation). In contrast to the prolonged survival and normal cardiac function observed in tamoxifen-treated $M y h$-TKO mice upto eight weeks after tamoxifen initiation, tamoxifentreated Myh-TKO mice challenged with isoproterenol exhibited death, dilated left ventricle and a significant contractile dysfunction only two weeks after tamoxifen initiation (Figure 5-4A, B, E). Histological analysis demonstrated loss of myofibrils and fibrosis (Figure 5-4C) and TEM analysis revealed severely damaged myocardium and mitochondrial cristae structure (Figure 5-4D), similar to observations in the Mcl-1deleted hearts. Further experiments are necessary to determine the mitochondrial respiration and mtDNA content of these hearts. No overt cardiac dysfunction or ultrastructural abnormalities is observed in the tamoxifen-treated $M c l-l^{\mathrm{wt} / \mathrm{wt}}, M y h$-Cre or Myh-DKO mice upon isoproterenol challenge (Figure 5-4).

Therefore, even though cell death is inhibited by BAX and BAK loss, Mcl-1 deletion in the heart revealed cardiac dysfunction and ultrastructural damage in response to isoproterenol challenge, indicating that MCL-1's function in promoting mitochondrial physiology and morphology is essential for normal cardiac function under stress. For further validation, we need to determine whether reconstitution of matrix-localized form of MCL- 1 to the Myh-TKO hearts via AAV delivery could rescue the cardiac dysfunction upon isoproterenol treatment. If this is the case, both functions of MCL-1 are necessary for cardiac homeostasis (Figure 5-5). 

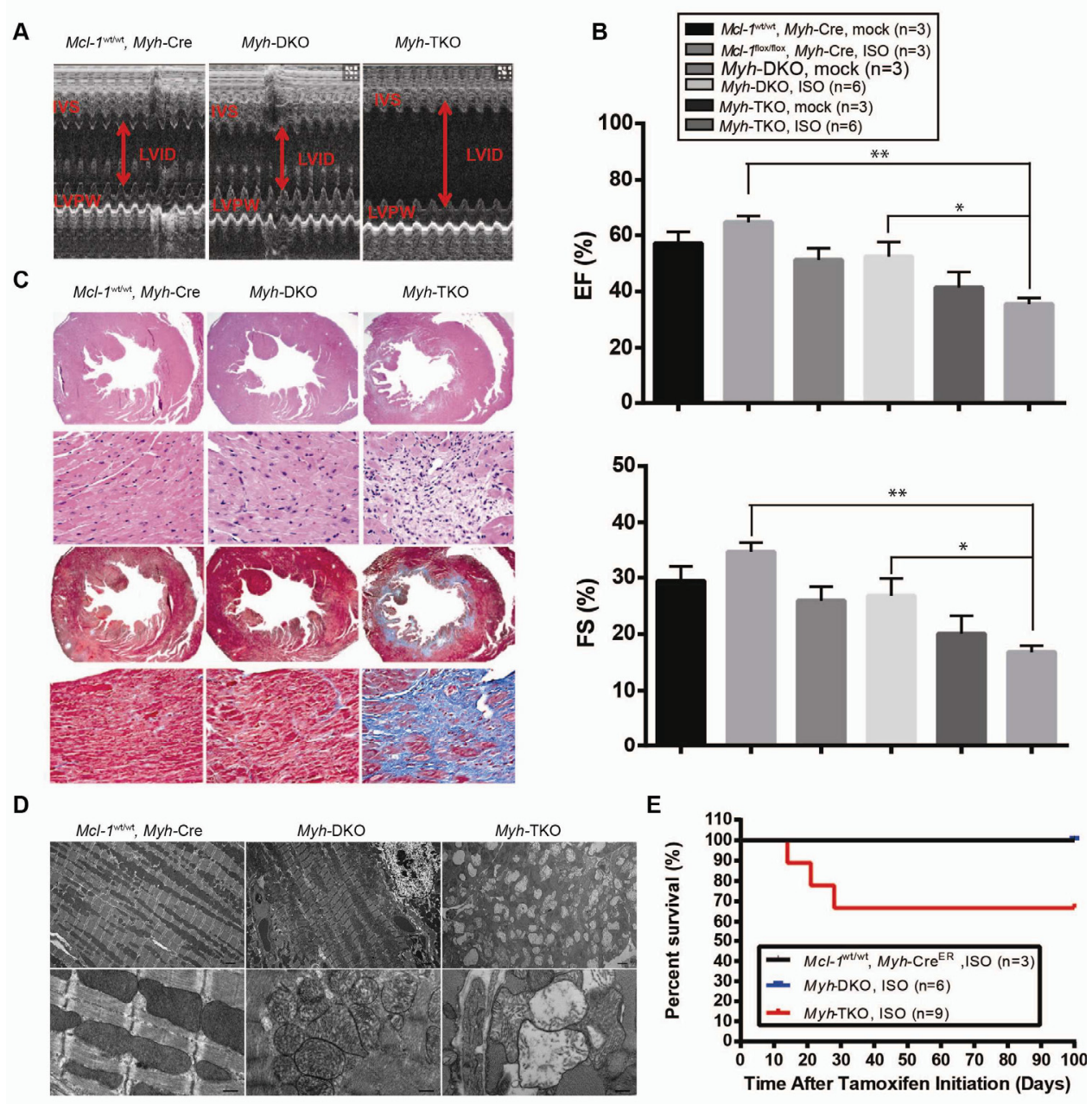

Figure 5-4. Isoproterenol challenge induces cardiac dilation and contractile dysfunction in Myh-TKO heart

Mice were treated with tamoxifen for five days, and then isoproterenol for one week. (A) Transthoracic echocardiographic (ECHO) studies were performed one week after isoproterenol initiation. M-mode images were recorded through interventricular septum (IVS) and left ventrical posterior wall (LVPW) at the papillary muscle level to get the left ventricle (LV) dimensions and IVS and LVPW thickness. (B) Contractility of heart as measured by ECHO for left ventricular (LV) ejection fraction (EF) and fraction shortening (FS). ${ }^{*} p<0.05, * * p<0.01$. The number of mice analyzed is indicated as (n). (C) H\&E-stained cross sections of the heart indicate dilated cardiomyopathy and a loss of myofibrils. Fibrosis in the cross-sections of the heart stained blue with the Masson's trichrome stain. (D) Transmission electron micrographs indicate disrupted myocardium and disorganized mitochondria with abnormal cristae structure in $M y h$-TKO heart after tamoxifen and isoproterenol treatment. Bars: top panels (lower magnification), $2 \mu \mathrm{m}$; bottom panels (higher magnification), $500 \mathrm{~nm}$. (E) Survival of $M c l-1^{\text {flox/flox }}, M y h$-Cre ${ }^{\mathrm{ER}}$, $M y h$-DKO, and $M y h$-TKO mice after tamoxifen and isoproterenol treatment. 


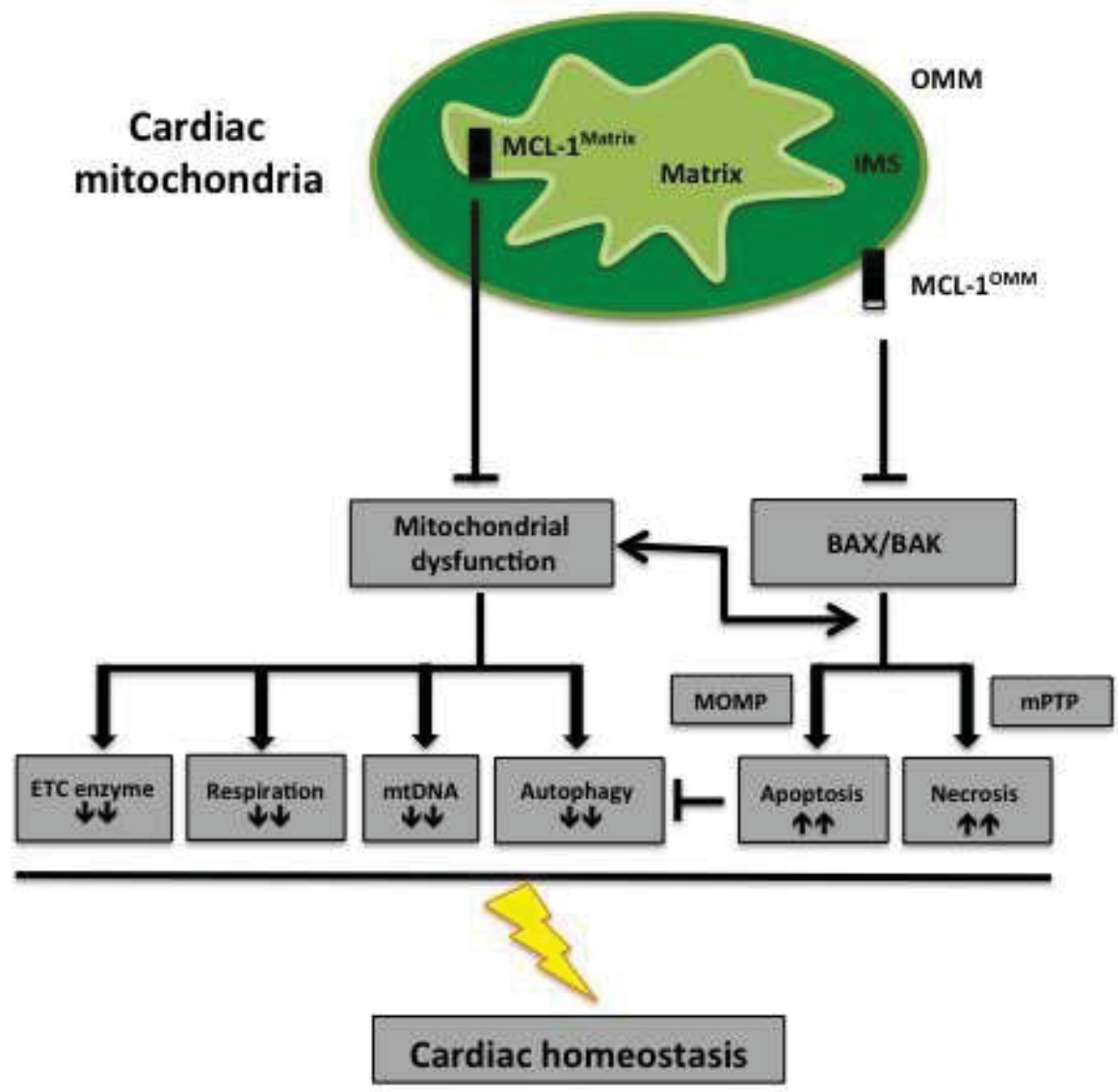

Figure 5-5. MCL-1 is essential for cardiac homeostasis

Both functions of MCL-1 are necessary for normal heart function. OMM-localized MCL1 inhibits cell death to promote cardiomyocytes survival, and matrix-localized MCL-1 facilitates mitochondrial physiology and morphology to maintain cardiac function. 


\section{LIST OF REFERENCES}

Abdelwahid E, Pelliniemi LJ, Niinikoski H, Simell O, Tuominen J, Rahkonen O, Jokinen E. 1999. Apoptosis in the pattern formation of the ventricular wall during mouse heart organogenesis. The Anatomical record 256: 208-217.

Adams JW, Sakata Y, Davis MG, Sah VP, Wang Y, Liggett SB, Chien KR, Brown JH, Dorn GW, 2nd. 1998. Enhanced Galphaq signaling: a common pathway mediates cardiac hypertrophy and apoptotic heart failure. Proceedings of the National Academy of Sciences of the United States of America 95: 10140-10145.

Adlam VJ, Harrison JC, Porteous CM, James AM, Smith RA, Murphy MP, Sammut IA. 2005. Targeting an antioxidant to mitochondria decreases cardiac ischemiareperfusion injury. FASEB journal : official publication of the Federation of American Societies for Experimental Biology 19: 1088-1095.

Akita Y, Koga Y, Iwanaga R, Wada N, Tsubone J, Fukuda S, Nakamura Y, Kato H. 2000. Fatal hypertrophic cardiomyopathy associated with an A8296G mutation in the mitochondrial tRNA(Lys) gene. Hum Mutat 15: 382.

Antozzi C, Zeviani M. 1997. Cardiomyopathies in disorders of oxidative metabolism. Cardiovasc Res 35: 184-199.

Arany Z, Novikov M, Chin S, Ma Y, Rosenzweig A, Spiegelman BM. 2006. Transverse aortic constriction leads to accelerated heart failure in mice lacking PPAR-gamma coactivator 1alpha. Proceedings of the National Academy of Sciences of the United States of America 103: 10086-10091.

Arbour N, Vanderluit JL, Le Grand JN, Jahani-Asl A, Ruzhynsky VA, Cheung EC, Kelly MA, MacKenzie AE, Park DS, Opferman JT et al. 2008. Mcl-1 is a key regulator of apoptosis during CNS development and after DNA damage. $J$ Neurosci 28: 6068-6078.

Asai K, Yang GP, Geng YJ, Takagi G, Bishop S, Ishikawa Y, Shannon RP, Wagner TE, Vatner DE, Homcy CJ et al. 1999. Beta-adrenergic receptor blockade arrests myocyte damage and preserves cardiac function in the transgenic G(salpha) mouse. The Journal of clinical investigation 104: 551-558.

Azfer A, Niu J, Rogers LM, Adamski FM, Kolattukudy PE. 2006. Activation of endoplasmic reticulum stress response during the development of ischemic heart disease. American journal of physiology Heart and circulatory physiology 291: H1411-1420.

Baines CP, Kaiser RA, Purcell NH, Blair NS, Osinska H, Hambleton MA, Brunskill EW, Sayen MR, Gottlieb RA, Dorn GW et al. 2005. Loss of cyclophilin D reveals a critical role for mitochondrial permeability transition in cell death. Nature 434: 658-662.

Balaban RS. 2002. Cardiac energy metabolism homeostasis: role of cytosolic calcium. Journal of molecular and cellular cardiology 34: 1259-1271.

Baldi A, Abbate A, Bussani R, Patti G, Melfi R, Angelini A, Dobrina A, Rossiello R, Silvestri F, Baldi F et al. 2002. Apoptosis and post-infarction left ventricular remodeling. Journal of molecular and cellular cardiology 34: 165-174.

Bardella C, Pollard PJ, Tomlinson I. 2011. SDH mutations in cancer. Biochimica et biophysica acta 1807: 1432-1443. 
Benit P, Beugnot R, Chretien D, Giurgea I, De Lonlay-Debeney P, Issartel JP, CorralDebrinski M, Kerscher S, Rustin P, Rotig A et al. 2003. Mutant NDUFV2 subunit of mitochondrial complex I causes early onset hypertrophic cardiomyopathy and encephalopathy. Hum Mutat 21: 582-586.

Beroukhim R, Mermel CH, Porter D, Wei G, Raychaudhuri S, Donovan J, Barretina J, Boehm JS, Dobson J, Urashima M et al. 2010. The landscape of somatic copynumber alteration across human cancers. Nature 463: 899-905.

Brandon M, Baldi P, Wallace DC. 2006. Mitochondrial mutations in cancer. Oncogene 25: 4647-4662.

Brocheriou V, Hagege AA, Oubenaissa A, Lambert M, Mallet VO, Duriez M, Wassef M, Kahn A, Menasche P, Gilgenkrantz H. 2000. Cardiac functional improvement by a human Bcl-2 transgene in a mouse model of ischemia/reperfusion injury. The journal of gene medicine 2: 326-333.

Bruick RK. 2000. Expression of the gene encoding the proapoptotic Nip3 protein is induced by hypoxia. Proceedings of the National Academy of Sciences of the United States of America 97: 9082-9087.

Bryant D, Becker L, Richardson J, Shelton J, Franco F, Peshock R, Thompson M, Giroir B. 1998. Cardiac failure in transgenic mice with myocardial expression of tumor necrosis factor-alpha. Circulation 97: 1375-1381.

Burstein HJ, Elias AD, Rugo HS, Cobleigh MA, Wolff AC, Eisenberg PD, Lehman M, Adams BJ, Bello CL, DePrimo SE et al. 2008. Phase II study of sunitinib malate, an oral multitargeted tyrosine kinase inhibitor, in patients with metastatic breast cancer previously treated with an anthracycline and a taxane. Journal of clinical oncology : official journal of the American Society of Clinical Oncology 26: 1810-1816.

Cai L, Kang YJ. 2001. Oxidative stress and diabetic cardiomyopathy: a brief review. Cardiovascular toxicology 1: 181-193.

Cai L, Li W, Wang G, Guo L, Jiang Y, Kang YJ. 2002. Hyperglycemia-induced apoptosis in mouse myocardium: mitochondrial cytochrome C-mediated caspase3 activation pathway. Diabetes 51: 1938-1948.

Capano M, Crompton M. 2006. Bax translocates to mitochondria of heart cells during simulated ischaemia: involvement of AMP-activated and p38 mitogen-activated protein kinases. The Biochemical journal 395: 57-64.

Cardinale D, Sandri MT, Colombo A, Colombo N, Boeri M, Lamantia G, Civelli M, Peccatori F, Martinelli G, Fiorentini C et al. 2004. Prognostic value of troponin I in cardiac risk stratification of cancer patients undergoing high-dose chemotherapy. Circulation 109: 2749-2754.

Cardinale D, Sandri MT, Martinoni A, Borghini E, Civelli M, Lamantia G, Cinieri S, Martinelli G, Fiorentini C, Cipolla CM. 2002. Myocardial injury revealed by plasma troponin I in breast cancer treated with high-dose chemotherapy. Annals of oncology : official journal of the European Society for Medical Oncology / ESMO 13: $710-715$.

Carvajal K, Moreno-Sanchez R. 2003. Heart metabolic disturbances in cardiovascular diseases. Arch Med Res 34: 89-99. 
Cavalli LR, Varella-Garcia M, Liang BC. 1997. Diminished tumorigenic phenotype after depletion of mitochondrial DNA. Cell growth \& differentiation : the molecular biology journal of the American Association for Cancer Research 8: 1189-1198.

Cella D, Wang M, Wagner L, Miller K. 2011. Survival-adjusted health-related quality of life (HRQL) among patients with metastatic breast cancer receiving paclitaxel plus bevacizumab versus paclitaxel alone: results from Eastern Cooperative Oncology Group Study 2100 (E2100). Breast cancer research and treatment 130: 855-861.

Certo M, Del Gaizo Moore V, Nishino M, Wei G, Korsmeyer S, Armstrong SA, Letai A. 2006. Mitochondria primed by death signals determine cellular addiction to antiapoptotic BCL-2 family members. Cancer cell 9: 351-365.

Chen H, Chomyn A, Chan DC. 2005a. Disruption of fusion results in mitochondrial heterogeneity and dysfunction. The Journal of biological chemistry 280: 2618526192.

Chen H, McCaffery JM, Chan DC. 2007. Mitochondrial fusion protects against neurodegeneration in the cerebellum. Cell 130: 548-562.

Chen H, Vermulst M, Wang YE, Chomyn A, Prolla TA, McCaffery JM, Chan DC. 2010. Mitochondrial fusion is required for mtDNA stability in skeletal muscle and tolerance of mtDNA mutations. Cell 141: 280-289.

Chen J, Hattori Y, Nakajima K, Eizawa T, Ehara T, Koyama M, Hirai T, Fukuda Y, Kinoshita M, Sugiyama A et al. 2006. Mitochondrial complex I activity is significantly decreased in a patient with maternally inherited type 2 diabetes mellitus and hypertrophic cardiomyopathy associated with mitochondrial DNA C3310T mutation: a cybrid study. Diabetes research and clinical practice 74: 148-153.

Chen L, Liu T, Tran A, Lu X, Tomilov AA, Davies V, Cortopassi G, Chiamvimonvat N, Bers DM, Votruba M et al. 2012. OPA1 mutation and late-onset cardiomyopathy: mitochondrial dysfunction and mtDNA instability. Journal of the American Heart Association 1: e003012.

Chen L, Willis SN, Wei A, Smith BJ, Fletcher JI, Hinds MG, Colman PM, Day CL, Adams JM, Huang DC. 2005b. Differential targeting of prosurvival Bcl-2 proteins by their BH3-only ligands allows complementary apoptotic function. Molecular cell 17: 393-403.

Chen M, Won DJ, Krajewski S, Gottlieb RA. 2002. Calpain and mitochondria in ischemia/reperfusion injury. The Journal of biological chemistry 277: 2918129186.

Chen Y, Liu Y, Dorn GW, 2nd. 2011. Mitochondrial fusion is essential for organelle function and cardiac homeostasis. Circ Res 109: 1327-1331.

Chen Z, Chua CC, Ho YS, Hamdy RC, Chua BH. 2001. Overexpression of Bcl-2 attenuates apoptosis and protects against myocardial I/R injury in transgenic mice. American journal of physiology Heart and circulatory physiology 280: H23132320.

Cheng EH, Wei MC, Weiler S, Flavell RA, Mak TW, Lindsten T, Korsmeyer SJ. 2001. BCL-2, BCL-X(L) sequester BH3 domain-only molecules preventing BAX- and BAK-mediated mitochondrial apoptosis. Mol Cell 8: 705-711. 
Chu TF, Rupnick MA, Kerkela R, Dallabrida SM, Zurakowski D, Nguyen L, Woulfe K, Pravda E, Cassiola F, Desai J et al. 2007. Cardiotoxicity associated with tyrosine kinase inhibitor sunitinib. Lancet 370: 2011-2019.

Ciciliot S, Schiaffino S. 2010. Regeneration of mammalian skeletal muscle. Basic mechanisms and clinical implications. Current pharmaceutical design 16: 906914.

Clarke MC, Littlewood TD, Figg N, Maguire JJ, Davenport AP, Goddard M, Bennett MR. 2008. Chronic apoptosis of vascular smooth muscle cells accelerates atherosclerosis and promotes calcification and medial degeneration. Circulation research 102: 1529-1538.

Claypool SM, Boontheung P, McCaffery JM, Loo JA, Koehler CM. 2008. The cardiolipin transacylase, tafazzin, associates with two distinct respiratory components providing insight into Barth syndrome. Molecular biology of the cell 19: 5143-5155.

Cory S, Adams JM. 2002. The Bcl2 family: regulators of the cellular life-or-death switch. Nat Rev Cancer 2: 647-656.

Da Cruz S, Xenarios I, Langridge J, Vilbois F, Parone PA, Martinou JC. 2003. Proteomic analysis of the mouse liver mitochondrial inner membrane. The Journal of biological chemistry 278: 41566-41571.

Dao Q, Krishnaswamy P, Kazanegra R, Harrison A, Amirnovin R, Lenert L, Clopton P, Alberto J, Hlavin P, Maisel AS. 2001. Utility of B-type natriuretic peptide in the diagnosis of congestive heart failure in an urgent-care setting. Journal of the American College of Cardiology 37: 379-385.

Das DAHaAM. 1991. Quantitative electron microscopic description of heart muscle cells.pdf>. Biochem J 280: 561-573.

Day CL, Chen L, Richardson SJ, Harrison PJ, Huang DC, Hinds MG. 2005. Solution structure of prosurvival Mcl-1 and characterization of its binding by proapoptotic BH3-only ligands. J Biol Chem 280: 4738-4744.

de Felipe P, Ryan MD. 2004. Targeting of proteins derived from self-processing polyproteins containing multiple signal sequences. Traffic 5: 616-626.

Detmer SA, Chan DC. 2007. Functions and dysfunctions of mitochondrial dynamics. Nature reviews Molecular cell biology 8: 870-879.

Di Napoli P, Taccardi AA, Grilli A, Felaco M, Balbone A, Angelucci D, Gallina S, Calafiore AM, De Caterina R, Barsotti A. 2003. Left ventricular wall stress as a direct correlate of cardiomyocyte apoptosis in patients with severe dilated cardiomyopathy. American heart journal 146: 1105-1111.

DiMauro S, Schon EA. 2003. Mitochondrial respiratory-chain diseases. The New England journal of medicine 348: 2656-2668.

Ding Q, He X, Hsu JM, Xia W, Chen CT, Li LY, Lee DF, Liu JC, Zhong Q, Wang X et al. 2007. Degradation of Mcl-1 by beta-TrCP mediates glycogen synthase kinase 3-induced tumor suppression and chemosensitization. Molecular and cellular biology 27: 4006-4017.

Ding Q, Huo L, Yang JY, Xia W, Wei Y, Liao Y, Chang CJ, Yang Y, Lai CC, Lee DF et al. 2008. Down-regulation of myeloid cell leukemia-1 through inhibiting Erk/Pin 1 pathway by sorafenib facilitates chemosensitization in breast cancer. Cancer research 68: 6109-6117. 
Diwan A, Wansapura J, Syed FM, Matkovich SJ, Lorenz JN, Dorn GW, 2nd. 2008. Nixmediated apoptosis links myocardial fibrosis, cardiac remodeling, and hypertrophy decompensation. Circulation 117: 396-404.

Dominov JA, Houlihan-Kawamoto CA, Swap CJ, Miller JB. 2001. Pro- and antiapoptotic members of the Bcl-2 family in skeletal muscle: a distinct role for Bcl-2 in later stages of myogenesis. Developmental dynamics : an official publication of the American Association of Anatomists 220: 18-26.

Dorn GW, 2nd. 2013. Mitochondrial dynamics in heart disease. Biochimica et biophysica acta 1833: 233-241.

Dorn GW, 2nd, Scorrano L. 2010. Two close, too close: sarcoplasmic reticulummitochondrial crosstalk and cardiomyocyte fate. Circulation research 107: 689699.

Doyama K, Fujiwara H, Fukumoto M, Tanaka M, Fujiwara Y, Oda T, Inada T, Ohtani S, Hasegawa K, Fujiwara T et al. 1996. Tumour necrosis factor is expressed in cardiac tissues of patients with heart failure. International journal of cardiology 54: $217-225$.

Dunkle A, Dzhagalov I, He YW. 2011. Cytokine-dependent and cytokine-independent roles for Mcl-1: genetic evidence for multiple mechanisms by which Mcl-1 promotes survival in primary T lymphocytes. Cell death \& disease 2: e214.

Dutka DP, Elborn JS, Delamere F, Shale DJ, Morris GK. 1993. Tumour necrosis factor alpha in severe congestive cardiac failure. British heart journal 70: 141-143.

Dzhagalov I, Dunkle A, He YW. 2008. The anti-apoptotic Bcl-2 family member Mcl-1 promotes T lymphocyte survival at multiple stages. Journal of immunology 181: 521-528.

Escudier B, Eisen T, Stadler WM, Szczylik C, Oudard S, Siebels M, Negrier S, Chevreau C, Solska E, Desai AA et al. 2007. Sorafenib in advanced clear-cell renal-cell carcinoma. The New England journal of medicine 356: 125-134.

Esposito LA, Melov S, Panov A, Cottrell BA, Wallace DC. 1999. Mitochondrial disease in mouse results in increased oxidative stress. Proc Natl Acad Sci U S A 96: 48204825.

Fernandez E, Siddiquee Z, Shohet RV. 2001. Apoptosis and proliferation in the neonatal murine heart. Developmental dynamics : an official publication of the American Association of Anatomists 221: 302-310.

Ferrari R, Bachetti T, Confortini R, Opasich C, Febo O, Corti A, Cassani G, Visioli O. 1995. Tumor necrosis factor soluble receptors in patients with various degrees of congestive heart failure. Circulation 92: 1479-1486.

Ferrari R, Censi, S., Mastrorilli, F., Boraso, A. 2003. Prognostic benefits of heart rate reduction in cardiovascular disease.pdf $>$. European Heart Journal Supplements 5: G10-14.

Fosslien E. 2003. Review: Mitochondrial medicine--cardiomyopathy caused by defective oxidative phosphorylation. Annals of clinical and laboratory science 33: 371-395.

Frost MT, Wang Q, Moncada S, Singer M. 2005. Hypoxia accelerates nitric oxidedependent inhibition of mitochondrial complex I in activated macrophages. American journal of physiology Regulatory, integrative and comparative physiology 288: R394-400. 
Fujise K, Zhang D, Liu J, Yeh ET. 2000. Regulation of apoptosis and cell cycle progression by MCL1. Differential role of proliferating cell nuclear antigen. $J$ Biol Chem 275: 39458-39465.

Galvez AS, Brunskill EW, Marreez Y, Benner BJ, Regula KM, Kirschenbaum LA, Dorn GW, 2nd. 2006. Distinct pathways regulate proapoptotic Nix and BNip3 in cardiac stress. The Journal of biological chemistry 281: 1442-1448.

Ganz WI, Sridhar KS, Ganz SS, Gonzalez R, Chakko S, Serafini A. 1996. Review of tests for monitoring doxorubicin-induced cardiomyopathy. Oncology 53: 461-470.

Garnier A, Fortin D, Delomenie C, Momken I, Veksler V, Ventura-Clapier R. 2003. Depressed mitochondrial transcription factors and oxidative capacity in rat failing cardiac and skeletal muscles. The Journal of physiology 551: 491-501.

Geng YJ, Ishikawa Y, Vatner DE, Wagner TE, Bishop SP, Vatner SF, Homcy CJ. 1999. Apoptosis of cardiac myocytes in Gsalpha transgenic mice. Circulation research 84: 34-42.

Germain M, Duronio V. 2007. The N terminus of the anti-apoptotic BCL-2 homologue MCL-1 regulates its localization and function. The Journal of biological chemistry 282: 32233-32242.

Germain M, Nguyen AP, Le Grand JN, Arbour N, Vanderluit JL, Park DS, Opferman JT, Slack RS. 2011. MCL-1 is a stress sensor that regulates autophagy in a developmentally regulated manner. The EMBO journal 30: 395-407.

Gilles S, Zahler S, Welsch U, Sommerhoff CP, Becker BF. 2003. Release of TNF-alpha during myocardial reperfusion depends on oxidative stress and is prevented by mast cell stabilizers. Cardiovascular research 60: 608-616.

Giroir BP, Johnson JH, Brown T, Allen GL, Beutler B. 1992. The tissue distribution of tumor necrosis factor biosynthesis during endotoxemia. The Journal of clinical investigation 90: 693-698.

Goldstein JD, Shanske S, Bruno C, Perszyk AA. 1999. Maternally inherited mitochondrial cardiomyopathy associated with a C-to-T transition at nucleotide 3303 of mitochondrial DNA in the tRNA(Leu(UUR)) gene. Pediatric and developmental pathology : the official journal of the Society for Pediatric Pathology and the Paediatric Pathology Society 2: 78-85.

Gottlieb RA, Burleson KO, Kloner RA, Babior BM, Engler RL. 1994. Reperfusion injury induces apoptosis in rabbit cardiomyocytes. J Clin Invest 94: 1621-1628.

Graham D, Huynh NN, Hamilton CA, Beattie E, Smith RA, Cocheme HM, Murphy MP, Dominiczak AF. 2009. Mitochondria-targeted antioxidant MitoQ10 improves endothelial function and attenuates cardiac hypertrophy. Hypertension 54: 322328.

Gross A, McDonnell JM, Korsmeyer SJ. 1999. BCL-2 family members and the mitochondria in apoptosis. Genes Dev 13: 1899-1911.

Guerra S, Leri A, Wang X, Finato N, Di Loreto C, Beltrami CA, Kajstura J, Anversa P. 1999. Myocyte death in the failing human heart is gender dependent. Circulation research 85: 856-866.

Gustafsson AB, Tsai JG, Logue SE, Crow MT, Gottlieb RA. 2004. Apoptosis repressor with caspase recruitment domain protects against cell death by interfering with Bax activation. The Journal of biological chemistry 279: 21233-21238. 
Hamada H, Suzuki M, Yuasa S, Mimura N, Shinozuka N, Takada Y, Suzuki M, Nishino T, Nakaya H, Koseki H et al. 2004. Dilated cardiomyopathy caused by aberrant endoplasmic reticulum quality control in mutant KDEL receptor transgenic mice. Molecular and cellular biology 24: 8007-8017.

Hamasaki A, Sendo F, Nakayama K, Ishida N, Negishi I, Nakayama K, Hatakeyama S. 1998. Accelerated neutrophil apoptosis in mice lacking A1-a, a subtype of the bcl2-related A1 gene. J Exp Med 188: 1985-1992.

Hanahan D, Weinberg RA. 2011. Hallmarks of cancer: the next generation. Cell 144: 646-674.

Haq R, Shoag J, Andreu-Perez P, Yokoyama S, Edelman H, Rowe GC, Frederick DT, Hurley AD, Nellore A, Kung AL et al. 2013. Oncogenic BRAF regulates oxidative metabolism via PGC1alpha and MITF. Cancer cell 23: 302-315.

Hauff KD, Hatch GM. 2006. Cardiolipin metabolism and Barth Syndrome. Progress in lipid research 45: 91-101.

Hein S, Arnon E, Kostin S, Schonburg M, Elsasser A, Polyakova V, Bauer EP, Klovekorn WP, Schaper J. 2003. Progression from compensated hypertrophy to failure in the pressure-overloaded human heart: structural deterioration and compensatory mechanisms. Circulation 107: 984-991.

Hengartner MO. 2000. The biochemistry of apoptosis. Nature 407: 770-776.

Hill MF, Palace VP, Kaur K, Kumar D, Khaper N, Singal PK. 2005. Reduction in oxidative stress and modulation of heart failure subsequent to myocardial infarction in rats. Experimental and clinical cardiology 10: 146-153.

Hinman LM, Blass JP. 1981. An NADH-linked spectrophotometric assay for pyruvate dehydrogenase complex in crude tissue homogenates. The Journal of biological chemistry 256: 6583-6586.

Hirota H, Chen J, Betz UA, Rajewsky K, Gu Y, Ross J, Jr., Muller W, Chien KR. 1999. Loss of a gp130 cardiac muscle cell survival pathway is a critical event in the onset of heart failure during biomechanical stress. Cell 97: 189-198.

Hochhauser E, Kivity S, Offen D, Maulik N, Otani H, Barhum Y, Pannet H, Shneyvays V, Shainberg A, Goldshtaub V et al. 2003. Bax ablation protects against myocardial ischemia-reperfusion injury in transgenic mice. American journal of physiology Heart and circulatory physiology 284: H2351-2359.

Huang CR, Yang-Yen HF. 2010. The fast-mobility isoform of mouse Mcl-1 is a mitochondrial matrix-localized protein with attenuated anti-apoptotic activity. FEBS letters 584: 3323-3330.

Huang HM, Huang CJ, Yen JJ. 2000. Mcl-1 is a common target of stem cell factor and interleukin-5 for apoptosis prevention activity via MEK/MAPK and PI-3K/Akt pathways. Blood 96: 1764-1771.

Ide T, Tsutsui H, Kinugawa S, Suematsu N, Hayashidani S, Ichikawa K, Utsumi H, Machida Y, Egashira K, Takeshita A. 2000. Direct evidence for increased hydroxyl radicals originating from superoxide in the failing myocardium. Circulation research 86: 152-157.

Ikeuchi M, Matsusaka H, Kang D, Matsushima S, Ide T, Kubota T, Fujiwara T, Hamasaki N, Takeshita A, Sunagawa K et al. 2005. Overexpression of mitochondrial transcription factor a ameliorates mitochondrial deficiencies and cardiac failure after myocardial infarction. Circulation 112: 683-690. 
Imahashi K, Schneider MD, Steenbergen C, Murphy E. 2004. Transgenic expression of Bcl-2 modulates energy metabolism, prevents cytosolic acidification during ischemia, and reduces ischemia/reperfusion injury. Circulation research 95: 734741.

Inuzuka H, Shaik S, Onoyama I, Gao D, Tseng A, Maser RS, Zhai B, Wan L, Gutierrez A, Lau AW et al. 2011. SCF(FBW7) regulates cellular apoptosis by targeting MCL1 for ubiquitylation and destruction. Nature 471: 104-109.

Jamil S, Sobouti R, Hojabrpour P, Raj M, Kast J, Duronio V. 2005. A proteolytic fragment of Mcl-1 exhibits nuclear localization and regulates cell growth by interaction with Cdk1. Biochem J 387: 659-667.

Jeremias I, Kupatt C, Martin-Villalba A, Habazettl H, Schenkel J, Boekstegers P, Debatin KM. 2000. Involvement of CD95/Apo1/Fas in cell death after myocardial ischemia. Circulation 102: 915-920.

Jouaville LS, Pinton P, Bastianutto C, Rutter GA, Rizzuto R. 1999. Regulation of mitochondrial ATP synthesis by calcium: evidence for a long-term metabolic priming. Proceedings of the National Academy of Sciences of the United States of America 96: 13807-13812.

Kapadia S, Lee J, Torre-Amione G, Birdsall HH, Ma TS, Mann DL. 1995. Tumor necrosis factor-alpha gene and protein expression in adult feline myocardium after endotoxin administration. The Journal of clinical investigation 96: 1042-1052.

Karmazyn M, Moffat MP. 1993. Role of $\mathrm{Na}+\mathrm{H}+$ exchange in cardiac physiology and pathophysiology: mediation of myocardial reperfusion injury by the $\mathrm{pH}$ paradox. Cardiovascular research 27: 915-924.

Kaufmann SH, Karp JE, Svingen PA, Krajewski S, Burke PJ, Gore SD, Reed JC. 1998. Elevated expression of the apoptotic regulator Mcl-1 at the time of leukemic relapse. Blood 91: 991-1000.

Kerkela R, Grazette L, Yacobi R, Iliescu C, Patten R, Beahm C, Walters B, Shevtsov S, Pesant S, Clubb FJ et al. 2006. Cardiotoxicity of the cancer therapeutic agent imatinib mesylate. Nature medicine 12: 908-916.

Kirshenbaum LA, Abdellatif M, Chakraborty S, Schneider MD. 1996. Human E2F-1 reactivates cell cycle progression in ventricular myocytes and represses cardiac gene transcription. Developmental biology 179: 402-411.

Kirshenbaum LA, de Moissac D. 1997. The bcl-2 gene product prevents programmed cell death of ventricular myocytes. Circulation 96: 1580-1585.

Kirshenbaum LA, Schneider MD. 1995. Adenovirus E1A represses cardiac gene transcription and reactivates DNA synthesis in ventricular myocytes, via alternative pocket protein- and p300-binding domains. The Journal of biological chemistry 270: 7791-7794.

Kobayashi S, Lee SH, Meng XW, Mott JL, Bronk SF, Werneburg NW, Craig RW, Kaufmann SH, Gores GJ. 2007. Serine 64 phosphorylation enhances the antiapoptotic function of Mcl-1. The Journal of biological chemistry 282: 1840718417.

Kockx MM, De Meyer GR, Muhring J, Jacob W, Bult H, Herman AG. 1998. Apoptosis and related proteins in different stages of human atherosclerotic plaques. Circulation 97: 2307-2315. 
Kohlhaas M, Liu T, Knopp A, Zeller T, Ong MF, Bohm M, O'Rourke B, Maack C. 2010. Elevated cytosolic $\mathrm{Na}+$ increases mitochondrial formation of reactive oxygen species in failing cardiac myocytes. Circulation 121: 1606-1613.

Kojima S, Hyakutake A, Koshikawa N, Nakagawara A, Takenaga K. 2010. MCL-1V, a novel mouse antiapoptotic MCL-1 variant, generated by RNA splicing at a noncanonical splicing pair. Biochemical and biophysical research communications 391: 492-497.

Konopleva M, Contractor R, Tsao T, Samudio I, Ruvolo PP, Kitada S, Deng X, Zhai D, Shi YX, Sneed T et al. 2006. Mechanisms of apoptosis sensitivity and resistance to the BH3 mimetic ABT-737 in acute myeloid leukemia. Cancer cell 10: 375 388.

Koopman WJ, Willems PH, Smeitink JA. 2012. Monogenic mitochondrial disorders. The New England journal of medicine 366: 1132-1141.

Koss B, Morrison J, Perciavalle RM, Singh H, Rehg JE, Williams RT, Opferman JT. 2013. Requirement for antiapoptotic MCL-1 in the survival of BCR-ABL Blineage acute lymphoblastic leukemia. Blood 122: 1587-1598.

Kostin S, Pool L, Elsasser A, Hein S, Drexler HC, Arnon E, Hayakawa Y, Zimmermann R, Bauer E, Klovekorn WP et al. 2003. Myocytes die by multiple mechanisms in failing human hearts. Circulation research 92: 715-724.

Kozopas KM, Yang T, Buchan HL, Zhou P, Craig RW. 1993. MCL1, a gene expressed in programmed myeloid cell differentiation, has sequence similarity to BCL2. Proc Natl Acad Sci U S A 90: 3516-3520.

Krajewski S, Bodrug S, Krajewska M, Shabaik A, Gascoyne R, Berean K, Reed JC. 1995. Immunohistochemical analysis of Mcl-1 protein in human tissues. Differential regulation of Mcl-1 and Bcl-2 protein production suggests a unique role for Mcl-1 in control of programmed cell death in vivo. Am J Pathol 146: 1309-1319.

Kroemer G. 2006. Mitochondria in cancer. Oncogene 25: 4630-4632.

Kroemer G, Galluzzi L, Brenner C. 2007. Mitochondrial membrane permeabilization in cell death. Physiol Rev 87: 99-163.

Kubasiak LA, Hernandez OM, Bishopric NH, Webster KA. 2002. Hypoxia and acidosis activate cardiac myocyte death through the Bcl-2 family protein BNIP3. Proceedings of the National Academy of Sciences of the United States of America 99: 12825-12830.

Kubli DA, Quinsay MN, Huang C, Lee Y, Gustafsson AB. 2008. Bnip3 functions as a mitochondrial sensor of oxidative stress during myocardial ischemia and reperfusion. American journal of physiology Heart and circulatory physiology 295: H2025-2031.

Kubota T, McTiernan CF, Frye CS, Slawson SE, Lemster BH, Koretsky AP, Demetris AJ, Feldman AM. 1997. Dilated cardiomyopathy in transgenic mice with cardiacspecific overexpression of tumor necrosis factor-alpha. Circulation research $\mathbf{8 1}$ : 627-635.

Kuznetsov AV, Margreiter R. 2009. Heterogeneity of mitochondria and mitochondrial function within cells as another level of mitochondrial complexity. Int J Mol Sci 10: 1911-1929. 
Latif N, Khan MA, Birks E, O'Farrell A, Westbrook J, Dunn MJ, Yacoub MH. 2000. Upregulation of the Bcl-2 family of proteins in end stage heart failure. Journal of the American College of Cardiology 35: 1769-1777.

Lee P, Sata M, Lefer DJ, Factor SM, Walsh K, Kitsis RN. 2003. Fas pathway is a critical mediator of cardiac myocyte death and MI during ischemia-reperfusion in vivo. American journal of physiology Heart and circulatory physiology 284: H456-463.

Lehman JJ, Kelly DP. 2002. Transcriptional activation of energy metabolic switches in the developing and hypertrophied heart. Clinical and experimental pharmacology \& physiology 29: 339-345.

Levine B, Kalman J, Mayer L, Fillit HM, Packer M. 1990. Elevated circulating levels of tumor necrosis factor in severe chronic heart failure. The New England journal of medicine 323: 236-241.

Levine B, Klionsky DJ. 2004. Development by self-digestion: molecular mechanisms and biological functions of autophagy. Developmental cell 6: 463-477.

Li F, Wang Y, Zeller KI, Potter JJ, Wonsey DR, O'Donnell KA, Kim JW, Yustein JT, Lee LA, Dang CV. 2005. Myc stimulates nuclearly encoded mitochondrial genes and mitochondrial biogenesis. Molecular and cellular biology 25: 6225-6234.

Li H, Wang J, Wilhelmsson H, Hansson A, Thoren P, Duffy J, Rustin P, Larsson NG. 2000. Genetic modification of survival in tissue-specific knockout mice with mitochondrial cardiomyopathy. Proc Natl Acad Sci U S A 97: 3467-3472.

Li J, Lee B, Lee AS. 2006. Endoplasmic reticulum stress-induced apoptosis: multiple pathways and activation of p53-up-regulated modulator of apoptosis (PUMA) and NOXA by p53. The Journal of biological chemistry 281: 7260-7270.

Li Y, Takemura G, Kosai K, Takahashi T, Okada H, Miyata S, Yuge K, Nagano S, Esaki M, Khai NC et al. 2004. Critical roles for the Fas/Fas ligand system in postinfarction ventricular remodeling and heart failure. Circulation research $\mathbf{9 5}$ : 627-636.

Li Z, Zhang T, Dai H, Liu G, Wang H, Sun Y, Zhang Y, Ge Z. 2007. Involvement of endoplasmic reticulum stress in myocardial apoptosis of streptozocin-induced diabetic rats. Journal of clinical biochemistry and nutrition 41: 58-67.

Lin L, Sharma VK, Sheu SS. 2007. Mechanisms of reduced mitochondrial Ca2+ accumulation in failing hamster heart. Pflugers Archiv : European journal of physiology 454: 395-402.

Lin M. 2011. Molecular imaging using positron emission tomography in colorectal cancer. Discovery medicine 11: 435-447.

Lindsten T, Ross AJ, King A, Zong WX, Rathmell JC, Shiels HA, Ulrich E, Waymire KG, Mahar P, Frauwirth K et al. 2000. The combined functions of proapoptotic Bcl-2 family members bak and bax are essential for normal development of multiple tissues. Molecular cell 6: 1389-1399.

Lyu YL, Kerrigan JE, Lin CP, Azarova AM, Tsai YC, Ban Y, Liu LF. 2007. Topoisomerase IIbeta mediated DNA double-strand breaks: implications in doxorubicin cardiotoxicity and prevention by dexrazoxane. Cancer research 67: 8839-8846.

Macheda ML, Rogers S, Best JD. 2005. Molecular and cellular regulation of glucose transporter (GLUT) proteins in cancer. Journal of cellular physiology 202: 654662. 
Magda D, Lecane P, Prescott J, Thiemann P, Ma X, Dranchak PK, Toleno DM, Ramaswamy K, Siegmund KD, Hacia JG. 2008. mtDNA depletion confers specific gene expression profiles in human cells grown in culture and in xenograft. BMC genomics 9: 521.

Maiuri MC, Le Toumelin G, Criollo A, Rain JC, Gautier F, Juin P, Tasdemir E, Pierron $\mathrm{G}$, Troulinaki K, Tavernarakis $\mathrm{N}$ et al. 2007. Functional and physical interaction between $\mathrm{Bcl}-\mathrm{X}(\mathrm{L})$ and a $\mathrm{BH} 3-$ like domain in Beclin-1. The EMBO journal 26: 2527-2539.

Mallat Z, Philip I, Lebret M, Chatel D, Maclouf J, Tedgui A. 1998. Elevated levels of 8iso-prostaglandin F2alpha in pericardial fluid of patients with heart failure: a potential role for in vivo oxidant stress in ventricular dilatation and progression to heart failure. Circulation 97: 1536-1539.

Maloyan A, Sayegh J, Osinska H, Chua BH, Robbins J. 2010. Manipulation of death pathways in desmin-related cardiomyopathy. Circulation research 106: 15241532.

Mannella CA. 2006. Structure and dynamics of the mitochondrial inner membrane cristae. Biochimica et biophysica acta 1763: 542-548.

Marin-Garcia J, Goldenthal MJ, Ananthakrishnan R, Pierpont ME. 2000. The complete sequence of mtDNA genes in idiopathic dilated cardiomyopathy shows novel missense and tRNA mutations. J Card Fail 6: 321-329.

Marin-Garcia J, Goldenthal MJ, Ananthakrishnan R, Pierpont ME, Fricker FJ, Lipshultz SE, Perez-Atayde A. 1996. Specific mitochondrial DNA deletions in idiopathic dilated cardiomyopathy. Cardiovasc Res 31: 306-313.

Matsui Y, Takagi H, Qu X, Abdellatif M, Sakoda H, Asano T, Levine B, Sadoshima J. 2007. Distinct roles of autophagy in the heart during ischemia and reperfusion: roles of AMP-activated protein kinase and Beclin 1 in mediating autophagy. Circulation research 100: 914-922.

Maurer U, Charvet C, Wagman AS, Dejardin E, Green DR. 2006. Glycogen synthase kinase-3 regulates mitochondrial outer membrane permeabilization and apoptosis by destabilization of MCL-1. Molecular cell 21: 749-760.

McBride HM, Neuspiel M, Wasiak S. 2006. Mitochondria: more than just a powerhouse. Curr Biol 16: R551-560.

McCormack JG, Halestrap AP, Denton RM. 1990. Role of calcium ions in regulation of mammalian intramitochondrial metabolism. Physiological reviews 70: 391-425.

Merante F, Tein I, Benson L, Robinson BH. 1994. Maternally inherited hypertrophic cardiomyopathy due to a novel T-to-C transition at nucleotide 9997 in the mitochondrial tRNA(glycine) gene. American journal of human genetics 55: 437446.

Michelfelder S, Trepel M. 2009. Adeno-Associated Viral Vectors and Their Redirection to Cell-Type Specific Receptors. 67: 29-60.

Millay DP, Sargent MA, Osinska H, Baines CP, Barton ER, Vuagniaux G, Sweeney HL, Robbins J, Molkentin JD. 2008. Genetic and pharmacologic inhibition of mitochondrial-dependent necrosis attenuates muscular dystrophy. Nature medicine 14: 442-447. 
Miller K, Wang M, Gralow J, Dickler M, Cobleigh M, Perez EA, Shenkier T, Cella D, Davidson NE. 2007. Paclitaxel plus bevacizumab versus paclitaxel alone for metastatic breast cancer. The New England journal of medicine 357: 2666-2676.

Miller KD, Chap LI, Holmes FA, Cobleigh MA, Marcom PK, Fehrenbacher L, Dickler M, Overmoyer BA, Reimann JD, Sing AP et al. 2005. Randomized phase III trial of capecitabine compared with bevacizumab plus capecitabine in patients with previously treated metastatic breast cancer. Journal of clinical oncology : official journal of the American Society of Clinical Oncology 23: 792-799.

Mills JR, Hippo Y, Robert F, Chen SM, Malina A, Lin CJ, Trojahn U, Wendel HG, Charest A, Bronson RT et al. 2008. mTORC1 promotes survival through translational control of Mcl-1. Proceedings of the National Academy of Sciences of the United States of America 105: 10853-10858.

Milner DJ, Mavroidis M, Weisleder N, Capetanaki Y. 2000. Desmin cytoskeleton linked to muscle mitochondrial distribution and respiratory function. $J$ Cell Biol 150: 1283-1298.

Milner DJ, Taffet GE, Wang X, Pham T, Tamura T, Hartley C, Gerdes AM, Capetanaki Y. 1999. The absence of desmin leads to cardiomyocyte hypertrophy and cardiac dilation with compromised systolic function. Journal of molecular and cellular cardiology 31: 2063-2076.

Milner DJ, Weitzer G, Tran D, Bradley A, Capetanaki Y. 1996. Disruption of muscle architecture and myocardial degeneration in mice lacking desmin. The Journal of cell biology 134: 1255-1270.

Minauro-Sanmiguel F, Wilkens S, Garcia JJ. 2005. Structure of dimeric mitochondrial ATP synthase: novel F0 bridging features and the structural basis of mitochondrial cristae biogenesis. Proceedings of the National Academy of Sciences of the United States of America 102: 12356-12358.

Miro O, Cardellach F, Barrientos A, Casademont J, Rotig A, Rustin P. 1998. Cytochrome c oxidase assay in minute amounts of human skeletal muscle using single wavelength spectrophotometers. Journal of neuroscience methods 80: 107-111.

Miyadera H, Shiomi K, Ui H, Yamaguchi Y, Masuma R, Tomoda H, Miyoshi H, Osanai A, Kita K, Omura S. 2003. Atpenins, potent and specific inhibitors of mitochondrial complex II (succinate-ubiquinone oxidoreductase). Proceedings of the National Academy of Sciences of the United States of America 100: 473-477.

Morais R, Zinkewich-Peotti K, Parent M, Wang H, Babai F, Zollinger M. 1994. Tumorforming ability in athymic nude mice of human cell lines devoid of mitochondrial DNA. Cancer research 54: 3889-3896.

Motoyama N, Wang F, Roth KA, Sawa H, Nakayama K, Nakayama K, Negishi I, Senju S, Zhang Q, Fujii S et al. 1995. Massive cell death of immature hematopoietic cells and neurons in Bcl-x-deficient mice. Science 267: 1506-1510.

Munoz-Pinedo C. 2012. Signaling pathways that regulate life and cell death: evolution of apoptosis in the context of self-defense. Adv Exp Med Biol 738: 124-143.

Murphy MP. 2009. How mitochondria produce reactive oxygen species. The Biochemical journal 417: 1-13. 
Murriel CL, Churchill E, Inagaki K, Szweda LI, Mochly-Rosen D. 2004. Protein kinase Cdelta activation induces apoptosis in response to cardiac ischemia and reperfusion damage: a mechanism involving $\mathrm{BAD}$ and the mitochondria. The Journal of biological chemistry 279: 47985-47991.

Nakagawa T, Shimizu S, Watanabe T, Yamaguchi O, Otsu K, Yamagata H, Inohara H, Kubo T, Tsujimoto Y. 2005. Cyclophilin D-dependent mitochondrial permeability transition regulates some necrotic but not apoptotic cell death. Nature 434: 652658.

Nakai A, Yamaguchi O, Takeda T, Higuchi Y, Hikoso S, Taniike M, Omiya S, Mizote I, Matsumura Y, Asahi M et al. 2007. The role of autophagy in cardiomyocytes in the basal state and in response to hemodynamic stress. Nature medicine 13: 619624.

Nakayama H, Chen X, Baines CP, Klevitsky R, Zhang X, Zhang H, Jaleel N, Chua BH, Hewett TE, Robbins $J$ et al. 2007. Ca2+- and mitochondrial-dependent cardiomyocyte necrosis as a primary mediator of heart failure. $J$ Clin Invest 117: 2431-2444.

Narula J, Haider N, Virmani R, DiSalvo TG, Kolodgie FD, Hajjar RJ, Schmidt U, Semigran MJ, Dec GW, Khaw BA. 1996. Apoptosis in myocytes in end-stage heart failure. The New England journal of medicine 335: 1182-1189.

Newmeyer DD, Ferguson-Miller S. 2003. Mitochondria: releasing power for life and unleashing the machineries of death. Cell 112: 481-490.

NHLBI. 2012. Morbidity \& Mortality: 2012 Chart Book on Cardiovascular, Lung and Blood Diseases.

Nijhawan D, Fang M, Traer E, Zhong Q, Gao W, Du F, Wang X. 2003. Elimination of Mcl-1 is required for the initiation of apoptosis following ultraviolet irradiation. Genes \& development 17: 1475-1486.

Nishida K, Yamaguchi O, Otsu K. 2008. Crosstalk between autophagy and apoptosis in heart disease. Circulation research 103: 343-351.

Okada K, Minamino T, Tsukamoto Y, Liao Y, Tsukamoto O, Takashima S, Hirata A, Fujita M, Nagamachi Y, Nakatani T et al. 2004. Prolonged endoplasmic reticulum stress in hypertrophic and failing heart after aortic constriction: possible contribution of endoplasmic reticulum stress to cardiac myocyte apoptosis. Circulation 110: 705-712.

Olivetti G, Abbi R, Quaini F, Kajstura J, Cheng W, Nitahara JA, Quaini E, Di Loreto C, Beltrami CA, Krajewski S et al. 1997. Apoptosis in the failing human heart. The New England journal of medicine 336: 1131-1141.

Oltersdorf T, Elmore SW, Shoemaker AR, Armstrong RC, Augeri DJ, Belli BA, Bruncko M, Deckwerth TL, Dinges J, Hajduk PJ et al. 2005. An inhibitor of Bcl-2 family proteins induces regression of solid tumours. Nature 435: 677-681.

Opferman JT. 2008. Apoptosis in the development of the immune system. Cell death and differentiation 15: 234-242.

Opferman JT, Iwasaki H, Ong CC, Suh H, Mizuno S, Akashi K, Korsmeyer SJ. 2005. Obligate role of anti-apoptotic MCL-1 in the survival of hematopoietic stem cells. Science 307: 1101-1104.

Opferman JT, Korsmeyer SJ. 2003. Apoptosis in the development and maintenance of the immune system. Nature immunology 4: 410-415. 
Opferman JT, Letai A, Beard C, Sorcinelli MD, Ong CC, Korsmeyer SJ. 2003. Development and maintenance of $\mathrm{B}$ and $\mathrm{T}$ lymphocytes requires antiapoptotic MCL-1. Nature 426: 671-676.

Otto Warburg FW, and Erwin Negelein. 1927. The metabolism of tumours in the body $J$ Gen Physiol 8: 11.

Pacak CA, Sakai Y, Thattaliyath BD, Mah CS, Byrne BJ. 2008. Tissue specific promoters improve specificity of AAV9 mediated transgene expression following intra-vascular gene delivery in neonatal mice. Genetic vaccines and therapy 6: 13.

Pattingre S, Tassa A, Qu X, Garuti R, Liang XH, Mizushima N, Packer M, Schneider MD, Levine B. 2005. Bcl-2 antiapoptotic proteins inhibit Beclin 1-dependent autophagy. Cell 122: 927-939.

Paumard P, Vaillier J, Coulary B, Schaeffer J, Soubannier V, Mueller DM, Brethes D, di Rago JP, Velours J. 2002. The ATP synthase is involved in generating mitochondrial cristae morphology. The EMBO journal 21: 221-230.

Pepper C, Lin TT, Pratt G, Hewamana S, Brennan P, Hiller L, Hills R, Ward R, Starczynski J, Austen B et al. 2008. Mcl-1 expression has in vitro and in vivo significance in chronic lymphocytic leukemia and is associated with other poor prognostic markers. Blood 112: 3807-3817.

Perciavalle RM, Stewart DP, Koss B, Lynch J, Milasta S, Bathina M, Temirov J, Cleland MM, Pelletier S, Schuetz JD et al. 2012. Anti-apoptotic MCL-1 localizes to the mitochondrial matrix and couples mitochondrial fusion to respiration. Nat Cell Biol 14: 575-583.

Petros AM, Olejniczak ET, Fesik SW. 2004. Structural biology of the Bcl-2 family of proteins. Biochimica et biophysica acta 1644: 83-94.

Picaud S, Kavanagh KL, Yue WW, Lee WH, Muller-Knapp S, Gileadi O, Sacchettini J, Oppermann U. 2011. Structural basis of fumarate hydratase deficiency. Journal of inherited metabolic disease 34: 671-676.

Poelmann RE, Gittenberger-de Groot AC. 2005. Apoptosis as an instrument in cardiovascular development. Birth defects research Part $C$, Embryo today : reviews 75: 305-313.

Porrello ER, Mahmoud AI, Simpson E, Hill JA, Richardson JA, Olson EN, Sadek HA. 2011. Transient regenerative potential of the neonatal mouse heart. Science 331: 1078-1080.

Potts MB, Vaughn AE, McDonough H, Patterson C, Deshmukh M. 2005. Reduced Apaf1 levels in cardiomyocytes engage strict regulation of apoptosis by endogenous XIAP. J Cell Biol 171: 925-930.

Powers SK, Jackson MJ. 2008. Exercise-induced oxidative stress: cellular mechanisms and impact on muscle force production. Physiological reviews 88: 1243-1276.

Print CG, Loveland KL, Gibson L, Meehan T, Stylianou A, Wreford N, de Kretser D, Metcalf D, Kontgen F, Adams JM et al. 1998. Apoptosis regulator bcl-w is essential for spermatogenesis but appears otherwise redundant. Proc Natl Acad Sci U S A 95: 12424-12431.

Procopio G, Verzoni E, Gevorgyan A, Mancin M, Pusceddu S, Catena L, Platania M, Guadalupi V, Martinetti A, Bajetta E. 2007. Safety and activity of sorafenib in different histotypes of advanced renal cell carcinoma. Oncology 73: 204-209. 
Rederstorff M, Castets P, Arbogast S, Laine J, Vassilopoulos S, Beuvin M, Dubourg O, Vignaud A, Ferry A, Krol A et al. 2011. Increased muscle stress-sensitivity induced by selenoprotein $\mathrm{N}$ inactivation in mouse: a mammalian model for SEPN1-related myopathy. PloS one 6: e23094.

Reeve JL, Szegezdi E, Logue SE, Ni Chonghaile T, O'Brien T, Ritter T, Samali A. 2007. Distinct mechanisms of cardiomyocyte apoptosis induced by doxorubicin and hypoxia converge on mitochondria and are inhibited by Bcl-xL. Journal of cellular and molecular medicine 11: 509-520.

Regula KM, Ens K, Kirshenbaum LA. 2002. Inducible expression of BNIP3 provokes mitochondrial defects and hypoxia-mediated cell death of ventricular myocytes. Circulation research 91: 226-231.

Rinkenberger JL, Horning S, Klocke B, Roth K, Korsmeyer SJ. 2000. Mcl-1 deficiency results in peri-implantation embryonic lethality. Genes Dev 14: 23-27.

Rosca MG, Hoppel CL. 2010. Mitochondria in heart failure. Cardiovascular research 88 : 40-50.

Rouslin W, Erickson JL, Solaro RJ. 1986. Effects of oligomycin and acidosis on rates of ATP depletion in ischemic heart muscle. The American journal of physiology 250: H503-508.

Saraste A, Pulkki K, Kallajoki M, Henriksen K, Parvinen M, Voipio-Pulkki LM. 1997. Apoptosis in human acute myocardial infarction. Circulation 95: 320-323.

Sathe P, Delconte RB, Souza-Fonseca-Guimaraes F, Seillet C, Chopin M, Vandenberg CJ, Rankin LC, Mielke LA, Vikstrom I, Kolesnik TB et al. 2014. Innate immunodeficiency following genetic ablation of Mcll in natural killer cells. Nature communications 5: 4539.

Scarabelli TM, Stephanou A, Pasini E, Comini L, Raddino R, Knight RA, Latchman DS. 2002. Different signaling pathways induce apoptosis in endothelial cells and cardiac myocytes during ischemia/reperfusion injury. Circulation research 90: $745-748$.

Scatena R. 2012. Mitochondria and cancer: a growing role in apoptosis, cancer cell metabolism and dedifferentiation. Advances in experimental medicine and biology 942: 287-308.

Schapira AH. 2012. Mitochondrial diseases. Lancet 379: 1825-1834.

Schlame M, Ren M. 2006. Barth syndrome, a human disorder of cardiolipin metabolism. FEBS letters 580: 5450-5455.

Schwartz A, Lee KS. 1962. Study of heart mitochondria and glycolytic metabolism in experimentally induced cardiac failure. Circulation research 10: 321-332.

Schwartz LM. 2008. Atrophy and programmed cell death of skeletal muscle. Cell death and differentiation 15: 1163-1169.

Schwickart M, Huang X, Lill JR, Liu J, Ferrando R, French DM, Maecker H, O'Rourke K, Bazan F, Eastham-Anderson J et al. 2010. Deubiquitinase USP9X stabilizes MCL1 and promotes tumour cell survival. Nature 463: 103-107.

Sedlak TW, Oltvai ZN, Yang E, Wang K, Boise LH, Thompson CB, Korsmeyer SJ. 1995. Multiple Bcl-2 family members demonstrate selective dimerizations with Bax. Proceedings of the National Academy of Sciences of the United States of America 92: 7834-7838. 
Senyo SE, Steinhauser ML, Pizzimenti CL, Yang VK, Cai L, Wang M, Wu TD, Guerquin-Kern JL, Lechene CP, Lee RT. 2013. Mammalian heart renewal by preexisting cardiomyocytes. Nature 493: 433-436.

Shiomi T, Tsutsui H, Matsusaka H, Murakami K, Hayashidani S, Ikeuchi M, Wen J, Kubota T, Utsumi H, Takeshita A. 2004. Overexpression of glutathione peroxidase prevents left ventricular remodeling and failure after myocardial infarction in mice. Circulation 109: 544-549.

Sohal DS, Nghiem M, Crackower MA, Witt SA, Kimball TR, Tymitz KM, Penninger JM, Molkentin JD. 2001. Temporally regulated and tissue-specific gene manipulations in the adult and embryonic heart using a tamoxifen-inducible Cre protein. Circulation research 89: 20-25.

Sommer JR, Waugh RA. 1978. Ultrastructure of heart muscle. Environ Health Perspect 26: $159-167$.

Song Y, Wang J, Li Y, Du Y, Arteel GE, Saari JT, Kang YJ, Cai L. 2005. Cardiac metallothionein synthesis in streptozotocin-induced diabetic mice, and its protection against diabetes-induced cardiac injury. Am J Pathol 167: 17-26.

Steimer DA, Boyd K, Takeuchi O, Fisher JK, Zambetti GP, Opferman JT. 2009. Selective roles for antiapoptotic MCL-1 during granulocyte development and macrophage effector function. Blood 113: 2805-2815.

Stewart DP, Koss B, Bathina M, Perciavalle RM, Bisanz K, Opferman JT. 2010. Ubiquitin-independent degradation of antiapoptotic MCL-1. Molecular and cellular biology 30: 3099-3110.

Strauss M, Hofhaus G, Schroder RR, Kuhlbrandt W. 2008. Dimer ribbons of ATP synthase shape the inner mitochondrial membrane. The EMBO journal 27: 11541160.

Supinski GS, Murphy MP, Callahan LA. 2009. MitoQ administration prevents endotoxininduced cardiac dysfunction. American journal of physiology Regulatory, integrative and comparative physiology 297: R1095-1102.

Swain SM, Whaley FS, Ewer MS. 2003. Congestive heart failure in patients treated with doxorubicin: a retrospective analysis of three trials. Cancer 97: 2869-2879.

Szegezdi E, Duffy A, O'Mahoney ME, Logue SE, Mylotte LA, O'Brien T, Samali A. 2006a. ER stress contributes to ischemia-induced cardiomyocyte apoptosis. Biochemical and biophysical research communications 349: 1406-1411.

Szegezdi E, Logue SE, Gorman AM, Samali A. 2006b. Mediators of endoplasmic reticulum stress-induced apoptosis. EMBO reports 7: 880-885.

Szymczak-Workman AL, Vignali KM, Vignali DA. 2012. Design and construction of 2A peptide-linked multicistronic vectors. Cold Spring Harbor protocols 2012: 199204.

Takagi H, Matsui Y, Hirotani S, Sakoda H, Asano T, Sadoshima J. 2007. AMPK mediates autophagy during myocardial ischemia in vivo. Autophagy 3: 405-407.

Takeuchi O, Fisher J, Suh H, Harada H, Malynn BA, Korsmeyer SJ. 2005. Essential role of BAX,BAK in B cell homeostasis and prevention of autoimmune disease. Proceedings of the National Academy of Sciences of the United States of America 102: 11272-11277. 
Tanaka M, Ito H, Adachi S, Akimoto H, Nishikawa T, Kasajima T, Marumo F, Hiroe M. 1994. Hypoxia induces apoptosis with enhanced expression of Fas antigen messenger RNA in cultured neonatal rat cardiomyocytes. Circulation research 75: 426-433.

Tannous P, Zhu H, Johnstone JL, Shelton JM, Rajasekaran NS, Benjamin IJ, Nguyen L, Gerard RD, Levine B, Rothermel BA et al. 2008. Autophagy is an adaptive response in desmin-related cardiomyopathy. Proceedings of the National Academy of Sciences of the United States of America 105: 9745-9750.

Terasaki F, Tanaka M, Kawamura K, Kanzaki Y, Okabe M, Hayashi T, Shimomura H, Ito T, Suwa M, Gong JS et al. 2001. A case of cardiomyopathy showing progression from the hypertrophic to the dilated form: association of Mt8348A-$>\mathrm{G}$ mutation in the mitochondrial tRNA(Lys) gene with severe ultrastructural alterations of mitochondria in cardiomyocytes. Japanese circulation journal $\mathbf{6 5}$ : 691-694.

Thomas RL, Roberts DJ, Kubli DA, Lee Y, Quinsay MN, Owens JB, Fischer KM, Sussman MA, Miyamoto S, Gustafsson AB. 2013. Loss of MCL-1 leads to impaired autophagy and rapid development of heart failure. Genes \& development 27: 1365-1377.

Thuerauf DJ, Marcinko M, Gude N, Rubio M, Sussman MA, Glembotski CC. 2006. Activation of the unfolded protein response in infarcted mouse heart and hypoxic cultured cardiac myocytes. Circulation research 99: 275-282.

Tiepolo T, Angelin A, Palma E, Sabatelli P, Merlini L, Nicolosi L, Finetti F, Braghetta P, Vuagniaux G, Dumont JM et al. 2009. The cyclophilin inhibitor Debio 025 normalizes mitochondrial function, muscle apoptosis and ultrastructural defects in Col6a1-/- myopathic mice. British journal of pharmacology 157: 1045-1052.

Torre-Amione G, Kapadia S, Lee J, Durand JB, Bies RD, Young JB, Mann DL. 1996. Tumor necrosis factor-alpha and tumor necrosis factor receptors in the failing human heart. Circulation 93: 704-711.

Toth A, Jeffers JR, Nickson P, Min JY, Morgan JP, Zambetti GP, Erhardt P. 2006. Targeted deletion of Puma attenuates cardiomyocyte death and improves cardiac function during ischemia-reperfusion. American journal of physiology Heart and circulatory physiology 291: H52-60.

Tsutsui H, Kinugawa S, Matsushima S. 2009. Mitochondrial oxidative stress and dysfunction in myocardial remodelling. Cardiovascular research 81: 449-456.

van Delft MF, Wei AH, Mason KD, Vandenberg CJ, Chen L, Czabotar PE, Willis SN, Scott CL, Day CL, Cory S et al. 2006. The BH3 mimetic ABT-737 targets selective Bcl-2 proteins and efficiently induces apoptosis via Bak/Bax if Mcl-1 is neutralized. Cancer cell 10: 389-399.

Vazquez F, Lim JH, Chim H, Bhalla K, Girnun G, Pierce K, Clish CB, Granter SR, Widlund HR, Spiegelman BM et al. 2013. PGC1alpha expression defines a subset of human melanoma tumors with increased mitochondrial capacity and resistance to oxidative stress. Cancer cell 23: 287-301.

Veis DJ, Sorenson CM, Shutter JR, Korsmeyer SJ. 1993. Bcl-2-deficient mice demonstrate fulminant lymphoid apoptosis, polycystic kidneys, and hypopigmented hair. Cell 75: 229-240. 
Von Hoff DD, Layard MW, Basa P, Davis HL, Jr., Von Hoff AL, Rozencweig M, Muggia FM. 1979. Risk factors for doxorubicin-induced congestive heart failure. Annals of internal medicine 91: 710-717.

Vuorinen K, Ylitalo K, Peuhkurinen K, Raatikainen P, Ala-Rami A, Hassinen IE. 1995. Mechanisms of ischemic preconditioning in rat myocardium. Roles of adenosine, cellular energy state, and mitochondrial F1F0-ATPase. Circulation 91: 28102818.

Wallace DC. 1999. Mitochondrial diseases in man and mouse. Science 283: 1482-1488. Animal models for mitochondrial disease. Methods Mol Biol 197: 3-54.

Wallace DC. 2012. Mitochondria and cancer. Nature Reviews Cancer 12: 685-698.

Wallace DC, Fan W. 2009. The pathophysiology of mitochondrial disease as modeled in the mouse. Genes \& development 23: 1714-1736.

Wang J, Wilhelmsson H, Graff C, Li H, Oldfors A, Rustin P, Bruning JC, Kahn CR, Clayton DA, Barsh GS et al. 1999a. Dilated cardiomyopathy and atrioventricular conduction blocks induced by heart-specific inactivation of mitochondrial DNA gene expression. Nat Genet 21: 133-137.

Wang JM, Chao JR, Chen W, Kuo ML, Yen JJ, Yang-Yen HF. 1999b. The antiapoptotic gene mcl-1 is up-regulated by the phosphatidylinositol 3-kinase/Akt signaling pathway through a transcription factor complex containing CREB. Molecular and cellular biology 19: 6195-6206.

Wang X, Bathina M, Lynch J, Koss B, Calabrese C, Frase S, Schuetz JD, Rehg JE, Opferman JT. 2013. Deletion of MCL-1 causes lethal cardiac failure and mitochondrial dysfunction. Genes \& development 27: 1351-1364.

Wang Z, Zhu T, Qiao C, Zhou L, Wang B, Zhang J, Chen C, Li J, Xiao X. 2005. Adenoassociated virus serotype 8 efficiently delivers genes to muscle and heart. Nat Biotechnol 23: 321-328.

Ward PS, Patel J, Wise DR, Abdel-Wahab O, Bennett BD, Coller HA, Cross JR, Fantin VR, Hedvat CV, Perl AE et al. 2010. The common feature of leukemia-associated IDH1 and IDH2 mutations is a neomorphic enzyme activity converting alphaketoglutarate to 2-hydroxyglutarate. Cancer cell 17: 225-234.

Warr MR, Shore GC. 2008. Unique biology of Mcl-1: therapeutic opportunities in cancer. Current molecular medicine 8: 138-147.

Wei G, Twomey D, Lamb J, Schlis K, Agarwal J, Stam RW, Opferman JT, Sallan SE, den Boer ML, Pieters R et al. 2006. Gene expression-based chemical genomics identifies rapamycin as a modulator of MCL1 and glucocorticoid resistance. Cancer Cell 10: 331-342.

Wei MC, Zong WX, Cheng EH, Lindsten T, Panoutsakopoulou V, Ross AJ, Roth KA, MacGregor GR, Thompson CB, Korsmeyer SJ. 2001. Proapoptotic BAX and BAK: a requisite gateway to mitochondrial dysfunction and death. Science 292: 727-730.

Weinberg F, Hamanaka R, Wheaton WW, Weinberg S, Joseph J, Lopez M, Kalyanaraman B, Mutlu GM, Budinger GR, Chandel NS. 2010. Mitochondrial metabolism and ROS generation are essential for Kras-mediated tumorigenicity. Proceedings of the National Academy of Sciences of the United States of America 107: 8788-8793. 
Weisleder N, Taffet GE, Capetanaki Y. 2004. Bcl-2 overexpression corrects mitochondrial defects and ameliorates inherited desmin null cardiomyopathy. Proceedings of the National Academy of Sciences of the United States of America 101: 769-774.

Wencker D. 2003. A mechanistic role for cardiac myocyte apoptosis in heart failure. Journal of Clinical Investigation 111: 1497-1504.

Whelan RS, Kaplinskiy V, Kitsis RN. 2010. Cell death in the pathogenesis of heart disease: mechanisms and significance. Annu Rev Physiol 72: 19-44.

Whelan RS, Konstantinidis K, Wei AC, Chen Y, Reyna DE, Jha S, Yang Y, Calvert JW, Lindsten T, Thompson CB et al. 2012. Bax regulates primary necrosis through mitochondrial dynamics. Proceedings of the National Academy of Sciences of the United States of America 109: 6566-6571.

Willis SN, Chen L, Dewson G, Wei A, Naik E, Fletcher JI, Adams JM, Huang DC. 2005. Proapoptotic Bak is sequestered by $\mathrm{Mcl}-1$ and $\mathrm{Bcl}-\mathrm{xL}$, but not Bcl-2, until displaced by BH3-only proteins. Genes \& development 19: 1294-1305.

Willis SN, Fletcher JI, Kaufmann T, van Delft MF, Chen L, Czabotar PE, Ierino H, Lee EF, Fairlie WD, Bouillet P et al. 2007. Apoptosis initiated when BH3 ligands engage multiple Bcl-2 homologs, not Bax or Bak. Science 315: 856-859.

Wirawan E, Vande Walle L, Kersse K, Cornelis S, Claerhout S, Vanoverberghe I, Roelandt R, De Rycke R, Verspurten J, Declercq W et al. 2010. Caspasemediated cleavage of Beclin-1 inactivates Beclin-1-induced autophagy and enhances apoptosis by promoting the release of proapoptotic factors from mitochondria. Cell death \& disease 1: e18.

Wise DR, DeBerardinis RJ, Mancuso A, Sayed N, Zhang XY, Pfeiffer HK, Nissim I, Daikhin E, Yudkoff M, McMahon SB et al. 2008. Myc regulates a transcriptional program that stimulates mitochondrial glutaminolysis and leads to glutamine addiction. Proceedings of the National Academy of Sciences of the United States of America 105: 18782-18787.

Wolfram JA, Donahue JK. 2013. Gene therapy to treat cardiovascular disease. Journal of the American Heart Association 2: e000119.

Wouters KA, Kremer LC, Miller TL, Herman EH, Lipshultz SE. 2005. Protecting against anthracycline-induced myocardial damage: a review of the most promising strategies. British journal of haematology 131: 561-578.

Xin M, Olson EN, Bassel-Duby R. 2013. Mending broken hearts: cardiac development as a basis for adult heart regeneration and repair. Nature reviews Molecular cell biology 14: 529-541.

Xu C, Bailly-Maitre B, Reed JC. 2005. Endoplasmic reticulum stress: cell life and death decisions. The Journal of clinical investigation 115: 2656-2664.

Yang JC, Haworth L, Sherry RM, Hwu P, Schwartzentruber DJ, Topalian SL, Steinberg SM, Chen HX, Rosenberg SA. 2003. A randomized trial of bevacizumab, an antivascular endothelial growth factor antibody, for metastatic renal cancer. The New England journal of medicine 349: 427-434.

Yang T, Kozopas KM, Craig RW. 1995. The intracellular distribution and pattern of expression of Mcl-1 overlap with, but are not identical to, those of Bcl-2. J Cell Biol 128: 1173-1184. 
Ye G, Metreveli NS, Ren J, Epstein PN. 2003. Metallothionein prevents diabetes-induced deficits in cardiomyocytes by inhibiting reactive oxygen species production. Diabetes 52: 777-783.

Yeh ET, Bickford CL. 2009. Cardiovascular complications of cancer therapy: incidence, pathogenesis, diagnosis, and management. Journal of the American College of Cardiology 53: 2231-2247.

Youle RJ, Strasser A. 2008. The BCL-2 protein family: opposing activities that mediate cell death. Nat Rev Mol Cell Biol 9: 47-59.

Yousefi S, Perozzo R, Schmid I, Ziemiecki A, Schaffner T, Scapozza L, Brunner T, Simon HU. 2006. Calpain-mediated cleavage of Atg5 switches autophagy to apoptosis. Nature cell biology 8: 1124-1132.

Yurkova N, Shaw J, Blackie K, Weidman D, Jayas R, Flynn B, Kirshenbaum LA. 2008. The cell cycle factor E2F-1 activates Bnip3 and the intrinsic death pathway in ventricular myocytes. Circulation research 102: 472-479.

Zhang D, Mott JL, Chang SW, Stevens M, Mikolajczak P, Zassenhaus HP. 2005. Mitochondrial DNA mutations activate programmed cell survival in the mouse heart. American journal of physiology Heart and circulatory physiology 288: H2476-2483.

Zhong Q, Gao W, Du F, Wang X. 2005. Mule/ARF-BP1, a BH3-only E3 ubiquitin ligase, catalyzes the polyubiquitination of Mcl-1 and regulates apoptosis. Cell 121: 10851095.

Zhou P, Qian L, Kozopas KM, Craig RW. 1997. Mcl-1, a Bcl-2 family member, delays the death of hematopoietic cells under a variety of apoptosis-inducing conditions. Blood 89: 630-643.

Zhu H, Tannous P, Johnstone JL, Kong Y, Shelton JM, Richardson JA, Le V, Levine B, Rothermel BA, Hill JA. 2007. Cardiac autophagy is a maladaptive response to hemodynamic stress. The Journal of clinical investigation 117: 1782-1793.

Zhu L, Yu Y, Chua BH, Ho YS, Kuo TH. 2001. Regulation of sodium-calcium exchange and mitochondrial energetics by Bcl-2 in the heart of transgenic mice. Journal of molecular and cellular cardiology 33: 2135-2144.

Zincarelli C, Soltys S, Rengo G, Rabinowitz JE. 2008. Analysis of AAV serotypes 1-9 mediated gene expression and tropism in mice after systemic injection. Mol Ther 16: $1073-1080$.

Zu XL, Guppy M. 2004. Cancer metabolism: facts, fantasy, and fiction. Biochemical and biophysical research communications 313: 459-465. 


\section{VITA}

Xi Wang was born in Zigong, Sichuan, China in 1981. In July of 2004, she completed a Bachelor Degree of Medicine in Chongqing Medical University, and then finished a Master Degree of Medicine at Medical School of Shanghai Jiaotong University in 2007. After one year of residency training in the Xinhua Hosptial affiliated to Medical School of Shanghai Jiaotong University, she enrolled in the IPBS program at the University of Tennessee Health Center in 2008. She joined the Opferman laboratory at

the St. Jude Children's Research Hospital in 2010. She is expected to graduate in December 2014.

\section{Publications}

Wang X, Bathina M, Lynch J, Koss B, Calabrese C, Frase S, Schuetz JD, Rehg JE, Opferman JT. 2013. Deletion of MCL-1 causes lethal cardiac failure and mitochondrial dysfunction. Genes \& development 27: 1351-1364.

Jin YY, Wang X*, Du J, Cao RM, Law HK, Wang JJ, Chen TX. 2014. Epstein-Barr virus induces the differentiation of semi-mature dendritic cells from cord blood monocytes.

Human immunology 75: 306-316. (* Equal contribution)

Li-Harms X, Milasta S, Lynch J, Wright C, Joshi A, Iyengar R, Neale G, Wang X, Wang YD, Prolla TA, Thompson JE, Opferman JT, Green DR, Schuetz J, Kundu M. 2014. Mito-protective autophagy is impaired in erythroid cells of aged mtDNA-mutator mice. Blood. (in press)

Yao CM, Han XH, Zhang YD, Zhang H, Jin YY, Cao RM, Wang X, Liu QH, Zhao W, Chen TX. 2013. Clinical characteristics and genetic profiles of 44 patients with severe combined immunodeficiency (SCID): report from Shanghai, China (2004-2011). Journal of clinical immunology 33: 526-539.

Wang JJ, Li YF, Jin YY, Wang X, Chen TX. 2012. Effects of Epstein-Barr virus on the development of dendritic cells derived from cord blood monocytes: an essential role for apoptosis. The Brazilian journal of infectious diseases : an official publication of the Brazilian Society of Infectious Diseases 16: 19-26.

Wang LL, Jin YY, Hao YQ, Wang JJ, Yao CM, Wang X, Cao RM, Zhang H, Chen Y, Chen TX. 2011. Distribution and clinical features of primary immunodeficiency diseases in Chinese children (2004-2009). Journal of clinical immunology 31: 297-308. 